University of Nebraska - Lincoln

DigitalCommons@University of Nebraska - Lincoln

\title{
$9-2008$
}

\section{Interface effects in spin-polarized metal/insulator layered structures}

\author{
Julian P. Velev \\ University of Nebraska-Lincoln, julian.velev@unl.edu \\ Peter A. Dowben \\ University of Nebraska at Lincoln, pdowben@unl.edu \\ Evgeny Y. Tsymbal \\ University of Nebraska at Lincoln, tsymbal@unl.edu \\ Stephen J. Jenkins \\ University of Cambridge, sjj24@cam.ac.uk
}

\author{
A. N. Caruso \\ University of Missouri, Kansas City, carusoan@umkc.edu
}

Follow this and additional works at: https://digitalcommons.unl.edu/physicstsymbal

Part of the Condensed Matter Physics Commons

Velev, Julian P.; Dowben, Peter A.; Tsymbal, Evgeny Y.; Jenkins, Stephen J.; and Caruso, A. N., "Interface effects in spin-polarized metal/insulator layered structures" (2008). Evgeny Tsymbal Publications. 21. https://digitalcommons.unl.edu/physicstsymbal/21

This Article is brought to you for free and open access by the Research Papers in Physics and Astronomy at DigitalCommons@University of Nebraska - Lincoln. It has been accepted for inclusion in Evgeny Tsymbal Publications by an authorized administrator of DigitalCommons@University of Nebraska - Lincoln. 
SURFACE SCIENCE REPORTS Volume: 63 Issue: 9 Pages: 400-425

doi:10.1016/j.surfrep.2008.06.002 Copyright (c) 2008 Elsevier B.V. Used by permission.

\title{
Interface Effects in Spin-Polarized Metal/Insulator Layered Structures
}

\author{
J. P. Velev, P. A. Dowben, and E. Y. Tsymbal \\ Department of Physics and Astronomy, \\ Nebraska Center for Materials and Nanoscience, \\ University of Nebraska, Lincoln, Nebraska 68588, USA \\ S. J. Jenkins \\ Department of Chemistry, University of Cambridge, Lensfield Road, \\ Cambridge CB2 1EW, United Kingdom \\ A. N. Caruso \\ Department of Physics, University of Missouri, Kansas City, Missouri 64110, USA
}

\begin{abstract}
Recent advances in thin-film deposition techniques, such as molecular beam epitaxy and pulsed laser deposition, have allowed for the manufacture of heterostructures with nearly atomically abrupt interfaces. Although the bulk properties of the individual heterostructure components may be well-known, often the heterostructures exhibit novel and sometimes unexpected properties due to interface effects. At heterostructure interfaces, lattice structure, stoichiometry, interface electronic structure (bonding, interface states, etc.), and symmetry all conspire to produce behavior different from the bulk constituents. This review discusses why knowledge of the electronic structure and composition at the interfaces is pivotal to the understanding of the properties of heterostructures, particularly the (spin polarized) electronic transport in (magnetic) tunnel junctions.
\end{abstract}

Key words: magnetic tunnel junctions, interface states, spin polarization, multiferroics PACS: 85.75-d, 73.20-r, 73.21.-b, 73.40-c

\section{Contents}

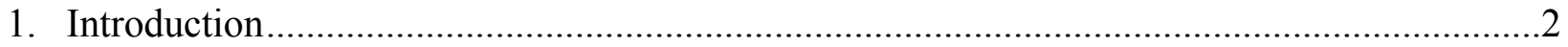

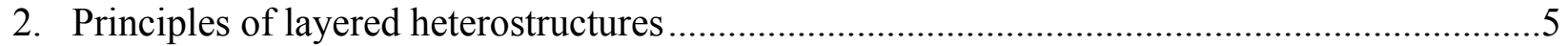

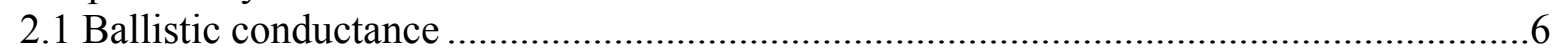

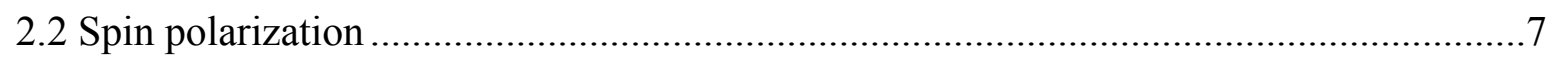

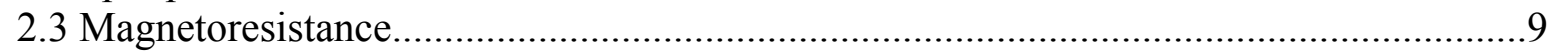

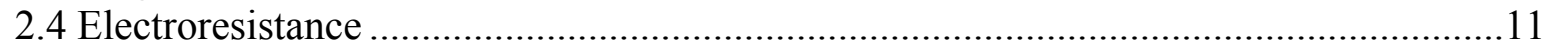

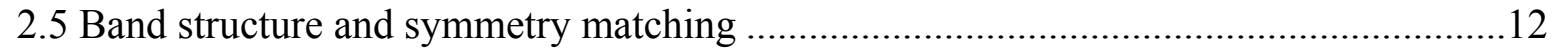

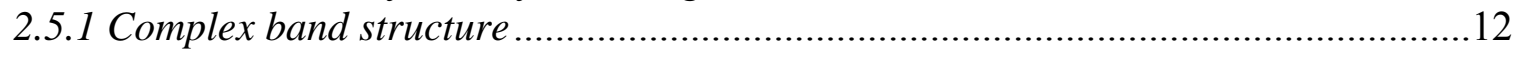

2.5.2 Interface matching: reflection and transmission coefficients .................................13

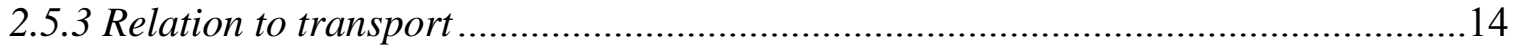




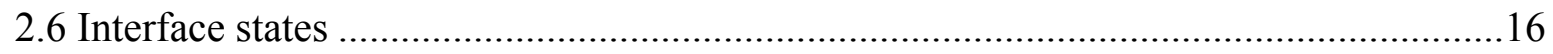

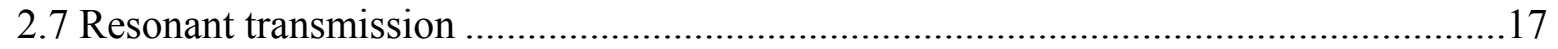

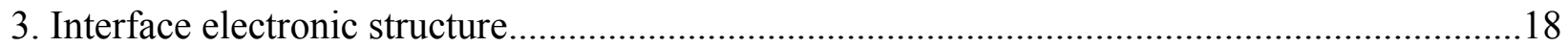

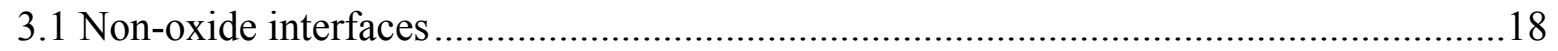

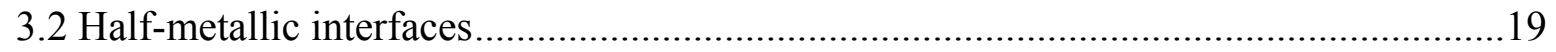

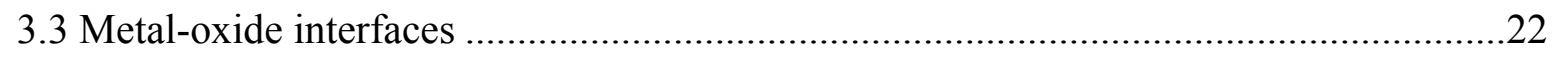

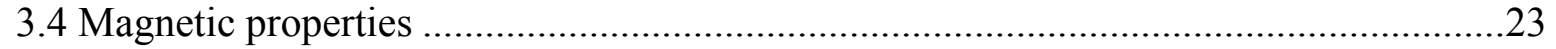

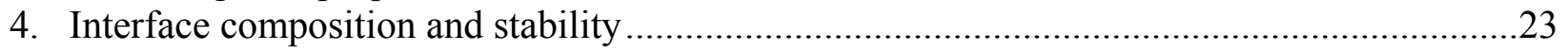

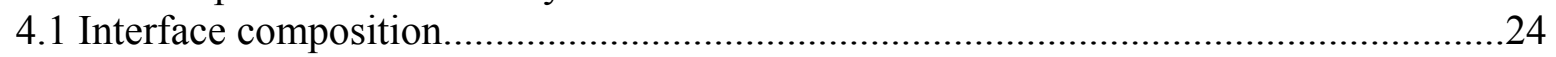

4.2 Interfaces with oxides: oxidation/reduction reactivity....................................................26

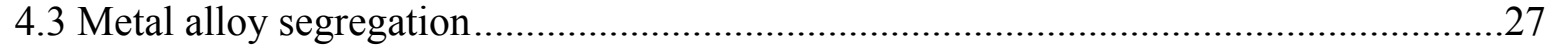

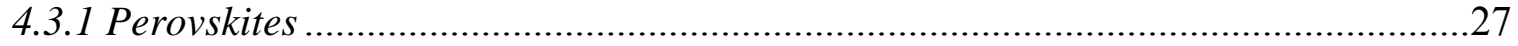

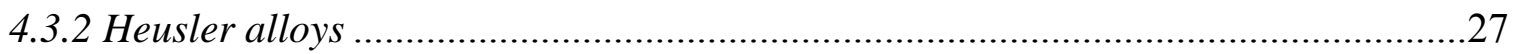

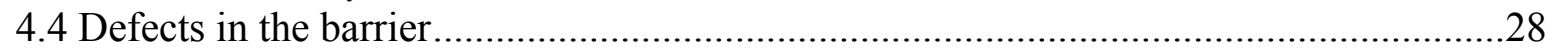

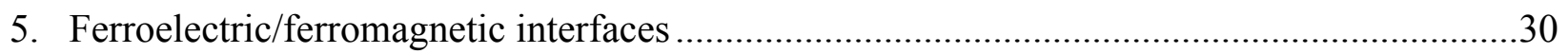

5.1 Ferroelectric tunnel junctions ................................................................................

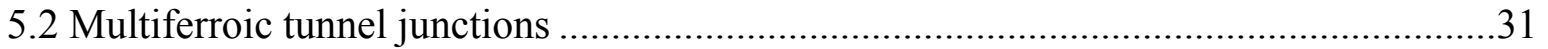

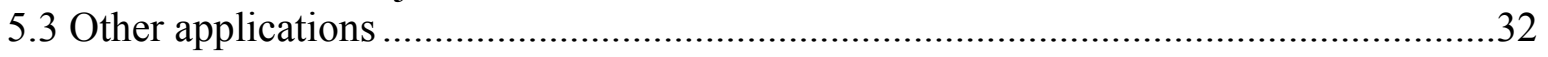

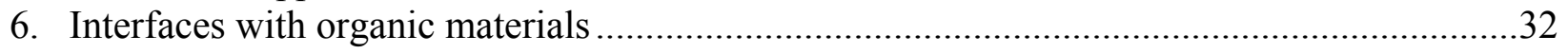

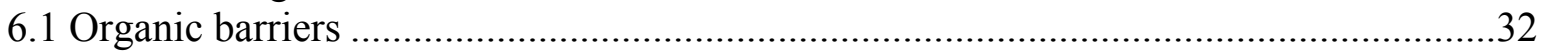

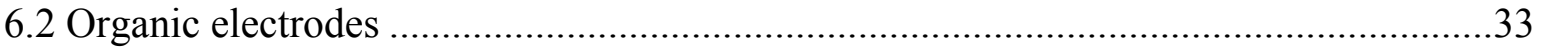

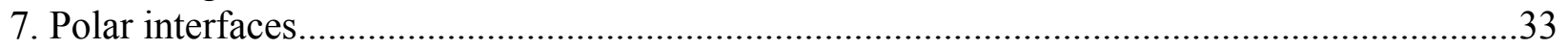

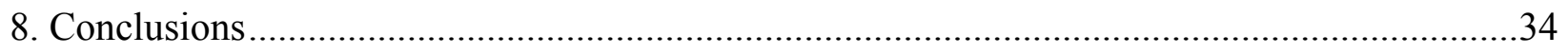

\section{Introduction}

Major advances in thin-film deposition techniques and characterization have enabled the development of materials which bring new functionalities to traditional devices or even novel device paradigms. In particular, advances in molecular beam epitaxy and pulsed laser deposition techniques have made it possible to manufacture layered heterostructures with nearly atomically abrupt epitaxial interfaces and individual layer thickness of the order of nanometers. In such nanoscale heterostructures, the ratio of interface to bulk material is comparable which, in addition to the high quality of the interfaces, ensures that the properties of these heterostructures are interface dominated.

The study of artificial materials such as layered heterostructures falls somewhat out of the scope of traditional disciplines. Traditional materials science has not generally been concerned with interfaces. Surface science is mainly focused on the interfaces between different phases, such as the solid and vacuum interface. The small dimensions of the heterostructures also require a microscopic approach on the level of individual atoms. These methods are a prerogative of condensed matter physics and quantum chemistry, which fields are normally not focused on applications. However, the development of nanoscale heterostructures has done much to revitalize all these fields and infuse them with the interdisciplinary field of surface and interface science [1-4]. One of the most prominent subfields has been the field of spin electronics (or spintronics), which promised to revolutionize electronics by introducing a new degree of freedom, the electron spin, to be exploited simultaneously along with the electron charge $[5,6]$. Although not all of the suggested potential for applications has been realized, the existing applications are already having a tremendous impact in information technology with applications such as high-density magnetic recording $[7,8]$ and magnetic random access memory (MRAM) $[9,10]$.

The task of spintronics from its onset has been to search for solutions beyond traditional electronics, which is reaching its natural scalability limit. Thus, spintronics is intentionally on the 'lookout' for emerging materials that bring new functionalities. A 
very broad range of materials have been considered as possible candidates for spintronics applications, such as ordinary semiconductors [11-13], ferromagnetic semiconductors [14-16], organic semiconductors [1719], single molecules [20,21], single molecular magnets $[22,23], \quad$ organic-based magnetic semiconductors [24,25], carbon nanotubes [26,27] and graphene [28,29]. Recently, thin-film ferroelectrics have aroused significant interest due to their technological application in ferroelectric random access memory (FERAM) devices [30,31]. These developments have broadened into a search for a new class of multifunctional spintronics materials, i.e., materials that can perform more than one task or that can be manipulated by several independent stimuli [3234]. Multifunctional materials exhibit two or more (coupled) ferroic orders, such as ferromagnetic, ferroelectric, or ferroelastic and are often referred to as multiferroic. The relative scarcity of single-phase multiferroic materials [35] is circumvented by the emerging field of artificial multiferroics that combine different ferroic materials in the same heterostructure $[36,37]$.

\section{(a) SV: GMR}

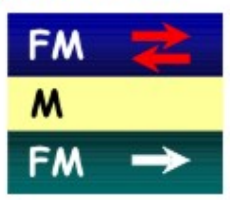

(c) FTJ: GER

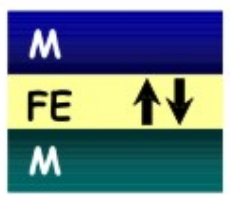

(b) MTJ: TMR

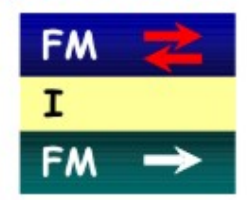

(d) MFTJ: 4-resistance

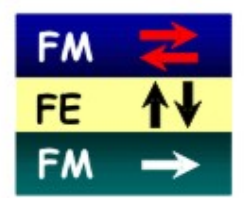

Fig. 1: Heterojunction structure and properties: (a) magnetic spin valve (SV), resistance change in magnetic field - giant magnetoresistance (GMR); (b) magnetic tunnel junction (MTJ), tunneling magnetoresistance (TMR); (c) ferroelectric tunnel junction (FTJ), resistance change in electric filed giant electroresistance (GER); and (d) multiferroic tunnel junction (MFTJ), resistance change in both electric and magnetic fields (4-Resistance). The ferromagnetic (FM), ferroelectric (FE), paramagnetic metal (M) and insulating (I) layers are indicated where appropriate.

At the heart of spintronics is the dependence of the physical properties of materials (in particular electron transport) on the electron spin. The scientific interest in spin-dependent transport originates from the work of
Mott [38] who realized that at low temperatures the electric current in ferromagnetic metals is a sum of two independent spin currents carried by majority- and minority-spin electrons. Much later, experiments by Tedrow and Meservey [39], who studied tunneling from a ferromagnet through an insulator into a superconductor, demonstrated the presence of nonvanishing spin polarization (SP) of the tunneling current. This discovery may be considered as the first demonstration of a functioning spintronics device. Four years later, Jullière observed the effect of tunneling magnetoresistance (TMR) in a magnetic tunnel junction (MTJ) [40], shown in Fig. 1b. Unfortunately, the values of TMR observed were small and not reproducible, hampered by problems in making extremely thin and pinhole-free insulating layers.

The onset of sudden increase of interest in layered heterostructures can be traced to the discovery of the interlayer exchange coupling (IEC) $[41,42]$. Interlayer exchange coupling takes place in magnetic multilayers in which ferromagnetic layers are separated by a nonmagnetic spacer. It can be conducting as well as insulating, as shown in Fig. 1a,b. Interlayer exchange coupling was first observed for metallic spacers [41] and was found to oscillate as a function of spacer thickness [42]. Experimental observation of the interlayer exchange coupling across an insulator has been much more challenging because producing a thin uniform insulating barrier is rather difficult. There are only a few reports of measurements of the coupling across a tunnel barrier [43-45]. The interlayer exchange coupling can be explained either in terms of the spin torque exerted by one ferromagnet on the other [46-48] or in terms of the induced density of states in the spacer by the ferromagnets $[49,50]$.

Antiferromagnetic interlayer exchange coupling in magnetic multilayers at certain thicknesses of the spacer led to the discovery of giant magnetoresistance (GMR), i.e. a large change in the resistance of the multilayer when its magnetization is altered from antiparallel to parallel by an external magnetic field [51,52]. GMR received a great deal of attention because of very large values of magnetoresistance (MR) (for reviews of GMR see Refs. 53- 60). The typical GMR values of tens of percent at room temperature and hundreds of percent at low temperatures were a dramatic improvement over anisotropic magnetoresistance (AMR) [61], the predecessor of GMR for magnetic recording applications. GMR is independent of the interlayer exchange coupling and in all later applications utilized the spin valve setup [62] in which one of the magnetic 
layers is 'pinned' by exchange bias to an antiferromagnet while the other is free to rotate in external magnetic field (Fig. 1a). In this case, the spacer is thick enough to break the exchange interaction between the ferromagnets so that their magnetizations can be switched independently. The discovery of GMR stimulated tremendous progress in the field of spin electronics and led to the 2007 Nobel Prize in Physics being awarded to Albert Fert and Peter Grünberg, the GMR pioneers.

The success of GMR in comparison to the earlier TMR experiments of Jullière was due to the realization that metallic spacers did not need to be very thin and could be achieved within the limits of the technology. This led to reliable observations of large and reproducible effect. However, GMR is due to band structure matching (Sec. 2.5) and is therefore limited in magnitude and restricted to a few favorable combinations of materials $(\mathrm{Co} / \mathrm{Cu}$ and $\mathrm{Fe} / \mathrm{Cr})$. Eventually, the area of spin-dependent tunneling was reinvented after Moodera et al. demonstrated a large and reproducible TMR effect in amorphous $\mathrm{Al}_{2} \mathrm{O}_{3}$ based MTJs [63]. The reported TMR values in $\mathrm{Al}_{2} \mathrm{O}_{3}$ based MTJs were gradually improved to $70 \%$ at room temperature [64]. More recently the interest in spin dependent transport dramatically increased due to the reports of large TMR values about $200 \%$ in crystalline $\mathrm{Fe} / \mathrm{MgO} / \mathrm{Fe}(001) \mathrm{MTJs}$ at room temperature $[65,66]$ following theoretical predictions of virtually infinite TMR in these systems [67,68]. For reviews of TMR and spin-dependent tunneling see Refs. 69-72, and Ref. 73 which focuses on $\mathrm{Fe} / \mathrm{MgO}$ MTJs.

Recently, there has been a lot of interest in magnetoresistive applications involving oxide heterostructures because of reports of half-metallic behavior in materials such as $\mathrm{CrO}_{2}, \mathrm{Fe}_{3} \mathrm{O}_{4}, \mathrm{NiMnSb}$, and $\mathrm{La}_{2 / 3} \mathrm{Sr}_{1 / 3} \mathrm{TiO}_{3}$. Half metals are materials in which one spin band (channel) has a gap around the Fermi energy and the other is partially filled [74]. This implies that the electric current in such materials should be fully spin-polarized, i.e. carried solely by electrons with a certain spin orientation. This property of half metals, if realized, opens tremendous possibilities for device applications such as spin filters and spin injectors (for review on half metals see Refs. 75,76). In addition, oxides exhibit a variety of interesting properties: most oxides are insulating but they can be (half) metals or semiconductors with the appropriate doping. Their lattice constant is determined primarily by the $\mathrm{O}$ sublattice, which allows the production of high quality epitaxial heterostructures. For a review of oxide spintronics see Ref. 77.

More recently, it was realized that the large number of degrees of freedom existing in transition-metal oxides (e.g. ferroelectricity and/or ferromagnetism) can be exploited to create additional functionalities not existing in conventional materials. For example, exciting prospects are offered by ferroelectric tunnel junctions (FTJ) in which a ferroelectric thin film is used as a barrier in a tunnel junction [78] (see Fig. 1c). In FTJs with different electrodes, a potential profile is asymmetric with respect to the polarization orientation in the ferroelectric layer, producing a giant electroresistance (GER) effect [79,80]. Indications of GER have been reported in experiments on epitaxial $\mathrm{Pt} / \mathrm{Pb}\left(\mathrm{Zr}_{0.52} \mathrm{Ti}_{0.48}\right) \mathrm{O}_{3} / \mathrm{SrRuO}_{3}$ junctions [81]. In addition, multiferroic tunnel junctions (MFTJ), shown in Fig. 1d, in which a ferroelectric thin film is used as a barrier in an MTJ, could change resistance in both electric and magnetic fields due to magnetization switching of the electrodes or polarization switching of the barrier. A different type of multiferroic tunnel junction, utilizing a bulk multiferroic $\mathrm{La}_{0.1} \mathrm{Bi}_{0.9} \mathrm{MnO}_{3}$ as a barrier, has shown four resistance states [82].

Another major challenge of spintronics is the injection and detection of highly polarized spin currents in semiconductors [83]. The simplest setup is to join together a ferromagnet with a non-magnetic semiconductor and inject spins across the interface. However, the degree of the achieved spin polarization in such a device was expected to be low because the resistance is dominated by the (nonmagnetic) semiconductor [84]. Tunneling injection in the ferromagnet-insulator-semiconductor setup allows this problem to be overcome due to matching the resistances while preserving the spin polarization [85]. In practice, the Schottky barrier may be used as such a tunneling barrier. It appears that by controlling the Schottky barrier width it is possible to achieve a sizable spin polarization of injected electrons from a ferromagnet into a semiconductor [12]. Recently, spin filtering using magnetic insulators as barriers was demonstrated using $\mathrm{EuO}$ [86] and $\mathrm{NiFe}_{2} \mathrm{O}_{4}$ [87].

All the above phenomena and applications are strongly dependent on the existence of interfaces between heterogeneous materials in layered heterostructures. The presence of interfaces is especially important in metal/insulator heterostructures due to interface states, surface electrostatic dipoles, and chemical bonding [88], which may change significantly the interface properties compared to the bulk further 
enhanced by the fact that interface electronic structure may dominate the electron tunneling.

Interface states are solutions of the Schrödinger equation localized at the interface. According to the Bloch theorem in a perfectly periodic solid, the wave function has the form $\phi(\mathbf{r})=e^{i \mathbf{k} \cdot \mathbf{r}} u(\mathbf{r})$, where $u(\mathbf{r})$ is a function with the periodicity of the lattice and $\mathbf{k}$ is a real wave vector, because only these solutions of the Schrödinger equation are normalizable at infinity. When the symmetry is broken by the presence of the interface, solutions with a complex component of the wave vector perpendicular to the interface, $k_{\perp}$, are possible. Propagating states in the metal can match the evanescent states in the insulator penetrating into the insulator and forming the so-called metal-induced gap states (MIGS). Matching of two decaying states on both sides of the interface produces an intrinsic interface state which decays in both directions away from the interface. However, if the interface state hybridizes with the bulk states such a state can propagate very far into the bulk, forming an interface resonance. Surface states are a particular type of interface state where evanescent states in the solid are matched to decaying exponents in the vacuum. Interface states have large density of states close to the interface, and so they can have important consequences for the band alignment at the interface, which determines the position of the Fermi energy with respect to the band gap of the insulator (semiconductor). For reviews see Refs. 89,90.

Surface dipoles have an electrostatic origin. The second law of thermodynamics requires that the electrochemical potentials on both sides of the interface are to be the same in equilibrium. When two materials with different work functions are brought in contact the alignment of the electrochemical potential produces a potential difference across the interface. This potential is screened by charges induced in the two materials, which cause the formation of a space-charged layer (accumulation of positive and negative charges on either side of the interface). In metals, due to high electron concentration, this uncompensated charge is screened within a few Ångstrom. In semiconductors the carrier concentration is much lower than in metals and consequently the charge screening occurs over a relative large distance (many lattice spacings) away from the interface, bending the electronic bands of the semiconductor and creating a Schottky barrier (charge depletion layer) [91].

Chemical bonding is another important property of metal-dielectric interfaces. In metals the valence electrons are itinerant, i.e. they form an electron gas. However, on the metal-dielectric interface metallic atoms may be close to atoms with strong electron affinity, resulting in valence electrons forming covalent or ionic bonds. This leads to a charge transfer across the interface, producing an atomic-scale dipole layer. Electric polarization of the chemical bonds across the interface can account for the interface dipole strength without regard to the actual distribution of gap states [92].

Overall, the interfacial phenomena influence greatly the properties of nanoscale heterostructures. This is especially important for spintronics where various device applications rely on ferromagnetic metal/nonmetal heterostructures. This review will focus on the properties of heterostructures, especially relevant to spintronics, which cannot be explained entirely on the basis of the bulk properties of the constituents. In particular, we will discuss interface atomic structure and stoichiometry; interface electronic structure, including chemical bonding and interface states; and symmetry considerations in the context of canonical and next generation materials interfaces. This review is not intended to cover the whole research field related to metal/nonmetal interfaces but illustrates the significance of the interface phenomena mentioned above using particular examples from our expertise in the light of major developments in the field.

\section{Principles of layered heterostructures}

The phenomenon of electron tunneling has been known since the advent of quantum mechanics, but only recently has it been utilized in practical devices. A tunnel junction, as first discussed by Frenkel [93], consists of two metal electrodes separated by a nanometer-thick insulating barrier layer (Fig. 1b). Although forbidden by classical physics, an electron is allowed to traverse a potential barrier that exceeds the electron's energy. The electron therefore has a finite probability of being found on the opposite side of the barrier. A famous example is electron tunneling in superconducting tunnel junctions, discovered by Giaever, that allowed measurement of important properties of superconductors [94]. In the 1970s, spindependent electron tunneling from ferromagnetic metal electrodes across an amorphous $\mathrm{Al}_{2} \mathrm{O}_{3}$ film was observed by Tedrow and Meservey [39]. The latter discovery led Jullière to propose and demonstrate a magnetic tunnel junction in which the tunneling current 
depends on the relative magnetization orientation of the two ferromagnetic electrodes [40], the phenomenon now known as tunneling magnetoresistance [63]. With the progress in ferroelectric thin film deposition a new field of multifunctional tunnel junctions has emerged [78]. Kohlstedt et al. demonstrated a prototype of ferroelectric tunnel junction in which the resistance exhibits a hysteretic behavior [81]. Gajek et al. fabricated a multiferroic tunnel junction in which resistance is controlled by both electric and magnetic fields [82]. New kinds of tunnel junctions may be very useful for various technological applications including spin-electronic devices such as magnetic field sensors for high-density recording and non-volatile random access memories (magnetic, ferroelectric, or multiferroic).

\subsection{Ballistic conductance}

Classical transport theory is concerned predominantly with macroscopic materials, the dimensions of which are much larger than the electron mean free path (of the order of nanometers in metals). Between injection and detection, the electron experiences a large number of scattering events, therefore, this transport regime is known as diffusive. In this regime all phase information in the wave function is lost and only its amplitude is calculatied. The wave function amplitude is related to the number of carriers and therefore to the current.

Recently, rapid developments in thin-film fabrication techniques have enabled the production of layered heterostructures, in which the material thickness in the direction perpendicular to the interfaces is of the order of nanometers. In such structures, the mean free path of the electron is comparable with the structure size. Thus, the carrier traverses the structure without scattering which is the definition of ballistic transport regime. In this regime correct treatment of electron transport it is required to keep track of the phase of the electron. The Landauer-Büttiker method allows to describe multi-terminal phase-coherent conductors in terms of simple concepts such as transmission and reflection probabilities, bypassing questions regarding the internal state of the conductor [95-97]. For illustration purposes we consider the two-terminal setup, shown in Fig. 2, in which the sample is assumed to be attached to two electrodes with chemical potentials $\mu_{L}$ and $\mu_{R}$ respectively. The current is proportional to the probability for transmission between the electrode summed over all energies for which there is an occupied state in the one electrode and a corresponding unoccupied state in the other electrode

$I=\frac{2 e}{h} \int_{\mu_{L}}^{\mu_{R}} T(E)\left(f_{L}(E)-f_{R}(E)\right) d E$

where $f$ are the Fermi-Dirac distribution functions in the electrodes and $T$ is the probability for the electron to be transmitted from the left to the right electrode. In the case of small applied bias (linear response), the current is proportional to the applied bias voltage $V$ and the conductance is given by

$G=\frac{I}{V}=\frac{2 e^{2}}{h} T\left(E_{F}\right)$,

where $V=\mu_{L}-\mu_{R}$. Only electrons at the Fermi energy participate in the transport. Conductance is proportional to the transmission probability times a conductance quantum $\left(h / e^{2}=12.9 \mathrm{k} \Omega\right)$. The factor of 2 reflects spin degeneracy, in spin-polarized materials the conductance is a sum over the spin channels.

(a)

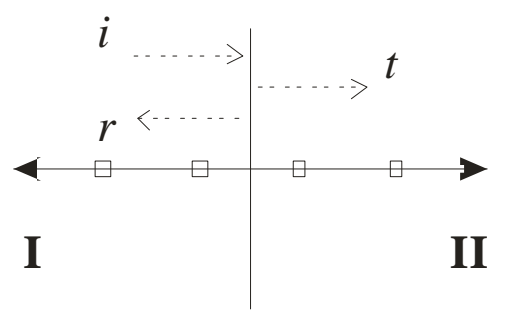

(b)

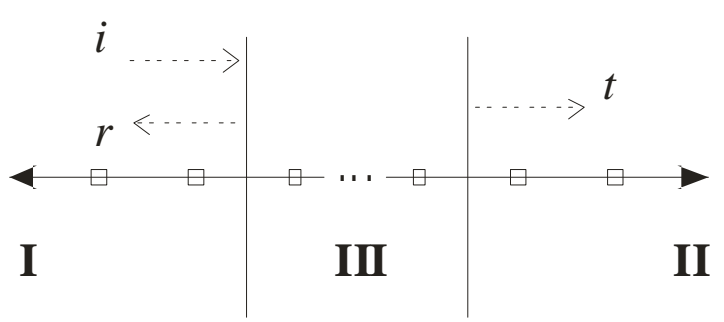

Fig. 2: Scattering setup: (a) interface reflection and transmission and (b) generalized reflection and transmission through a finite size scattering region. The incoming state (i) is partially reflected back $(r)$ and transmitted to the other size $(t)$. The generalized transmission setup is equivalent to the two-probe setup in which a sample is attached to semiinfinite electrodes.

Eq. (2) can be used in this form in real space for systems that lack periodicity in the plane perpendicular 
to the current: objects such as nanowires, nanotubes, or molecular junctions. If the system dimensions in the plane perpendicular to the current are much larger than the dimensions along the current it is convenient to employ a reciprocal space representation. Layered heterostructures, such as spin valves and tunnel junctions fall in this category.

The wave function of a periodic system is indexed by the wave vector $\mathbf{k}$, restricted to the first Brillouin zone of the reciprocal lattice. In layered structures, the periodicity is broken in the direction perpendicular to the interface but it is often periodic in the interface plane. Thus, the wave function can be written in mixed $\left(\mathbf{k}_{\|}, Z\right)$ representation. In this case

$T=\sum_{\mathbf{k}_{\|}, \mathbf{k}_{\|}^{\prime}} \sum_{i, j} T\left(\mathbf{k}_{\|}, i ; \mathbf{k}_{\|}^{\prime}, j\right)$,

where each individual term represents the probability for transmission from state $\mathbf{k}_{\|}, i$ in the left electrode to state $\mathbf{k}_{\|}^{\prime}, j$ in the right electrode. If there is no scattering then Eq. (3) leads to a great simplification because the tunneling problem can now be solved for each $\mathbf{k}_{\|}$independently. Almost all available theoretical work assumes epitaxial systems, in which the above mentioned simplifications apply. In contrast, most experimental work is done on amorphous barriers like $\mathrm{AlO}_{\mathrm{x}}$ and polycrystalline 'electrodes' where the transverse wave vector is not conserved in the process of tunneling. This causes problems in comparing theoretical and experimental results and in making predictions regarding decisive effects controlling spindependent tunneling. Fortunately, a lot of experimental effort has been directed towards smaller, more defectfree, epitaxial systems to which theoretical models apply. At the same time theory has advanced in the direction of more realistic models, including treatment of disorder. There are two ways to deal with disorder in modeling electron and spin transport - consider large supercells $[98,99]$ or use mean-field techniques such as the coherent potential approximation $[100,101]$.

The traditional view of transport as current carried by independent electrons fails to account for incoherent scattering events such as electron-electron, electronphonon, and electron-magnon scattering. In coherent transport, therefore, localized states carry no current because they are decoupled from the propagating states. However, the above-mentioned scattering processes would effectively introduce this coupling enabling the localized states to contribute to the transport on equal footing. The Bardeen approach to tunneling takes into account the transmission probability through the barrier, disregarding the mechanism of localized states filling. This can be used to describe the tunneling contribution of localized states [102].

\subsection{Spin polarization}

In ferromagnetic materials the spin degeneracy of electronic states is lifted and conductance becomes spin-dependent. One of the important characteristics of a ferromagnetic material is the degree of its spin polarization. This characteristic is not, however, uniquely defined and depends on what particular spindependent property of the ferromagnet is measured. In addition, the measured spin polarization may depend on other properties of the system used in the experiment (e.g., the interface between the ferromagnet and insulator in magnetic tunnel junctions).

One of the broadly-used definitions of the spin polarization involves the electronic density of states (DOS). Electronic bands in ferromagnetic metals are exchange-split and consequently the DOS is different for majority- and minority-spin electrons, $N_{\uparrow}(E)$ and $N_{\downarrow}(E)$, respectively. Since only carriers at the Fermi energy, $E_{F}$, participate in transport, the DOS at the Fermi energy is relevant

$$
N_{\sigma}=\frac{1}{(2 \pi)^{3}} \sum_{\alpha} \int \frac{d S_{F}^{\alpha \sigma}}{v_{\mathbf{k} \alpha \sigma}},
$$

where $\sigma$ is the spin index $(\sigma=\uparrow$ or $\downarrow$ ), the integration is performed over all the sheets of the Fermi surface $d S_{F}^{\alpha \sigma}, v_{\mathbf{k} \alpha \sigma}$ is the Fermi velocity, and a sum is taken over all bands $\alpha$. The spin polarization of the DOS at the Fermi energy is defined as follows

$P_{N}=\frac{N_{\uparrow}-N_{\downarrow}}{N_{\uparrow}+N_{\downarrow}}$.

The spin polarization defined by Eq. (5) may however be inappropriate, as far as transport properties are concerned. Indeed, the current density involves ferromagnet characteristics which cannot be entirely described by the DOS, such as the velocity of electrons 
and the transport relaxation time (in the diffusive transport regime). Therefore, a more relevant definition associated with transport is the spin polarization of the current

$$
P_{J}=\frac{J_{\uparrow}-J_{\downarrow}}{J_{\uparrow}+J_{\downarrow}},
$$

where $\quad J_{\uparrow}$ and $J_{\downarrow}$ are current densities corresponding to majority and minority-spin electrons, which are assumed to carry currents in two parallel channels.

In the ballistic transport regime [103] the current densities are uniquely defined by the electronic structure and are given by the projection of the Fermi surface onto the transport direction $\hat{\mathbf{n}}$ (see, e.g., Refs. $58,104,105)$

$$
J_{\sigma}^{b a l} \propto \sum_{\alpha} \int \hat{\mathbf{n}} \cdot d \mathbf{S}_{F}^{\alpha \sigma} .
$$

This quantity is also known as the number of conduction channels [97] which is the number of states available at the Fermi energy. Therefore, the spin polarization (6), in this case, is proportional to the difference in the number of states available for conductance in the two spin channels.

In the diffusive transport regime, for crystals of cubic or higher symmetry containing a low concentration of scatterers, the Boltzmann equation within a relaxation time approximation gives

$$
J_{\sigma}^{\text {diff }} \propto \sum_{\alpha} \int \tau_{\mathbf{k} \alpha \sigma} \mathbf{v}_{\mathbf{k} \alpha \sigma} \cdot d \mathbf{S}_{F}^{\alpha \sigma}
$$

Here $\tau_{\mathbf{k} \alpha \sigma}$ is the relaxation time, which depends on the particular type of scatterers (defects or impurities) and the density of electronic states in the crystal. The relaxation time is spin-dependent and hence cannot be ignored in the definition of the spin polarization in the diffusive transport regime. There has been much effort invested in the understanding of spin-dependent transport in bulk ferromagnets [106-109], and in GMR structures [53-60,110].

Eqs. (5), (6) together with Eqs. (4), (7), (8) give us three completely different definitions of the spin polarization. In definition (5) the DOS is determined as a sum over the Fermi surface of $1 / v_{F}$ and applies best to spin-polarized electron spectroscopies such as spin- polarized photoemission and inverse photoemission. Since $d$-electrons are localized and consequently the Fermi velocity $v_{F}$ of the $d$-electrons is very low, the spin polarization is mainly determined by the exchange splitting of the $d$ bands. We should note however that matrix elements which determine the transition probability of electron photoemission from a oneelectron orbital to a free state may be spin-dependent and hence may play a role in the magnitude of the spin polarization as determined from photoemission experiments.

In definition (6) with the current density given by Eq. (7) $J_{\sigma}^{b a l}$ counts the number of states at the Fermi surface and the spin polarization is determined by the difference in the number of Bloch states at the Fermi energy. This definition is relevant to Andreev reflection experiments [105,111].

Finally, the diffusive transport spin polarization (Eqs. (6) and (8)) contains a sum over the Fermi surface weighted by the Fermi velocity weighted by the relaxation time. Since dispersive s-like bands have high velocity they largely contribute to conductivity (8), while the $d$ bands provide final states for scattering of the $s$ electrons [38]. Due to exchange splitting of the $d$ bands, the probability of scattering into these states is proportional to their density, so that the scattering rates are spin-dependent. Although this picture is too simplified, in view of the strong hybridization between the $s$ and $d$ states, it forms a useful basis for a qualitative understanding of the spin-dependent conduction in the diffusive transport regime (see Ref. 58 for further discussion).

Neither of the three definitions given above are fully related to the spin polarization of the tunneling current measured in experiments by Tedrow and Meservey [39,69] on tunneling from a ferromagnet through an insulator into a superconductor. The results of these experiments were, first, interpreted in terms of the spin-dependent density of states, i.e. Eq. (5). It was found however, that there is inconsistency between the measured and predicted values of the spin polarization. Experimentally it was observed that the spin polarization of the tunneling conductance from all the $3 d$ ferromagnetic metals and their alloys is positive, which implies that the majority-spin electrons tunnel more efficiently than their minority-spin counterpart. This is in a contradiction with the bulk band structure, at least for the two ferromagnetic metals $\mathrm{Co}$ and $\mathrm{Ni}$, which have the dominant contribution of the minority spins at the Fermi energy causing the respective spin 
polarization of the DOS, Eq. (5), to be negative. This fact was later explained by Stearns [112], who assumed that the most dispersive bands of 'itinerant' electrons provide essentially all the tunneling current. Assuming that conductance is proportional only to the DOS of the itinerant electrons the spin polarization becomes

$P_{k}=\frac{k_{\uparrow}-k_{\downarrow}}{k_{\uparrow}+k_{\downarrow}}$,

where $k_{\uparrow}$ and $k_{\downarrow}$ are the Fermi wave vectors of the majority- and minority-spin itinerant electrons. Using a realistic band structure of $\mathrm{Fe}$ and $\mathrm{Ni}$, Stearns was able to explain experimental values (measured at that time) of the tunneling spin polarization for these ferromagnets.

Later, Slonczewski [46] generalized this free electron model to include explicitly a tunneling barrier and demonstrated that in the general case the tunneling polarization is expressed

$P_{\text {tun }}=P_{k} \frac{\kappa^{2}-k_{\uparrow} k_{\downarrow}}{\kappa^{2}+k_{\uparrow} k_{\downarrow}}$,

where $\kappa$ is the decay rate of the wave function in the barrier region. For a high potential barrier $\left(\kappa \gg k_{\uparrow}, k_{\downarrow}\right)$ one recovers Eq. (9). Eq. (10) suggests that the spin polarization of the tunneling current depends not only on the properties of ferromagnetic electrodes alone $\left(k_{\uparrow}\right.$ and $k_{\downarrow}$ ) but also on the barrier height entering Eq. (10) through $\kappa$.

Slonczewski's formula (10) for the tunneling spin polarization can be written in a different way

$P_{\text {tun }}=\frac{T_{\uparrow}-T_{\downarrow}}{T_{\uparrow}+T_{\downarrow}}$,

where $T_{\uparrow}$ and $T_{\downarrow}$ are given by

$T_{\sigma}=\frac{4 \kappa k_{\sigma}}{\kappa^{2}+k_{\sigma}^{2}}$.

The physical interpretation of $T_{\sigma}$ is the spin-dependent transmission probability from the ferromagnetic electrode to the barrier across the interface [73]. Thus, the tunneling spin polarization involves explicitly properties of the interface between the ferromagnet and the insulator.

The expression for the spin polarization has form (11) if the transmission coefficient can be decoupled into the product of two interface transmission functions $T_{L}^{\sigma}\left(\mathbf{k}_{\|}\right)$and $T_{R}^{\sigma}\left(\mathbf{k}_{\|}\right)$characterizing left and right interfaces respectively and the exponential decay factor $[73,113]$

$T_{\sigma}\left(\mathbf{k}_{\|}\right)=T_{L}^{\sigma}\left(\mathbf{k}_{\|}\right) e^{-2 \kappa d} T_{R}^{\sigma}\left(\mathbf{k}_{\|}\right)$,

where $d$ is the insulator thickness. This is possible provided that the barrier is sufficiently thick (no multiple scattering) and if there is only one dominant evanescent state controlling the transmission through the insulator [113]. Thus, it is important to realize that in experiments on spin-dependent tunneling (injection) the relevant spin polarization depends on more than just the spin polarized electrode but also involves properties of the insulator and the ferromagnet/nonmetal interface and the barrier layer.

Overall, from the above discussion, it is worth noting that we do not always measure a polarization that follows the usual definition Eq. (5). As we have just noted, there are corrections for the Fermi velocity $v_{\uparrow, \downarrow}$ and electron relaxation $\tau_{\uparrow, \downarrow}$. Even measurements of the polarization that are Fermi-velocity and relaxation-time independent, as in the case of spin-polarized photoemission and inverse photoemission, may still depend on the wave vector and details of the interface band structure. Thus quoted experimental polarization must be treated with caution and needs to be interpreted in the context of particular experiment.

\subsection{Magnetoresistance}

Layered heterostructures, such as spin valves (Fig. 1a) and magnetic tunnel junctions (Fig. 1b), consist of magnetic electrodes separated by a spacer layer. The magnetic electrode means any conducting material which exhibits a finite spin polarization at the Fermi energy, usually ferromagnetic, but ferrimagnetic and canted antiferromagnetic may also be considered. The magnetization of the electrodes can be switched independently. A reversal of the magnetic orientation of the electrodes from antiparallel to parallel by applied 
magnetic field produces a change in the electrical resistance of the heterostructure. Magnetoresistance (MR) is normally quantified by the ratio ${ }^{1}$

$$
\mathrm{MR}=\frac{R_{A P}-R_{P}}{R_{P}}=\frac{G_{P}-G_{A P}}{G_{A P}},
$$

where $R_{P}$ and $R_{A P}$ are resistances, and $G_{P}$ and $G_{A P}$ are conductances measured for the parallel and antiparallel magnetization of the electrodes.

The origin of MR stems from the fact that electrons preserve their spin while traversing the spacer layer, which creates imbalance in the electric current carried by electrons with different spin projections. In the parallel configuration the electrons can tunnel to the same states on the other side of the barrier, while in the antiparallel configuration this might not be possible due to band structure mismatch (Fig. 3). Thus, the resistance of the antiparallel configuration is typically higher than the resistance of the parallel configuration.

This type of resistance change in an external magnetic field was first observed in magnetic metallic multilayers and became known as giant magnetoresistance. GMR requires that the Fermi surface of the spacer matches one of the spin channels very well, so that the resistance of at least of one of the spin channels in the parallel configuration is lower than that of the antiparallel. Propagating states on both sides of the interface are matched i.e. GMR is a (real) band structure matching effect.

The corresponding effect in magnetic tunnel junctions is known as tunneling magnetoresistance. TMR depends on the complex Fermi surface of the insulator, which provides rates of decay for the different states in the insulator. At the interface, propagating states in the electrodes are matched to decaying states in the barrier i.e. TMR is a complex band structure matching effect.

\footnotetext{
${ }^{1}$ This definition is known as 'optimistic' because the ratio can be infinite. An alternative 'pessimistic' definition is $\mathrm{MR}=\left(G_{P}-G_{A P}\right) /\left(G_{P}+G_{A P}\right)$ according to which the ratio is limited to $\pm 100 \%$.
}

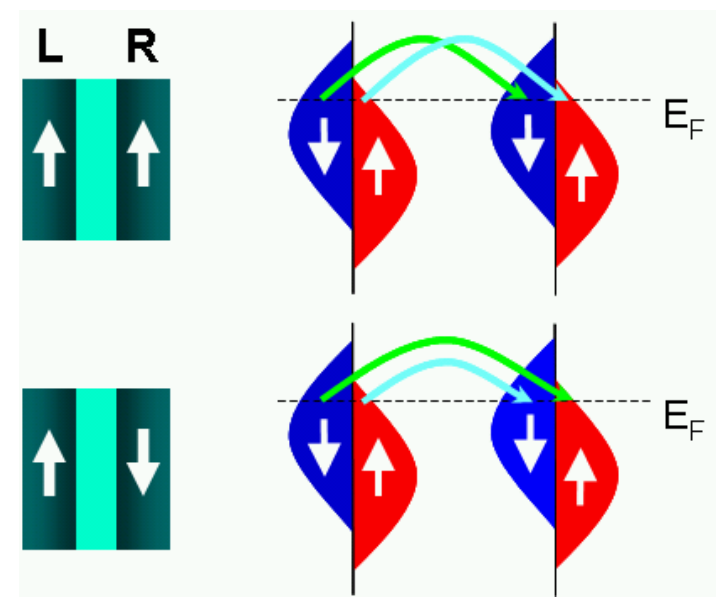

Fig. 3: A magnetic heterojunction characterized by parallel or antiparallel magnetization of the left (L) and right (R) ferromagnetic electrodes (left panels) and schematic representation of tunneling transitions between exchangesplit spin bands in the ferromagnetic electrodes (right panels).

The relationship between TMR and the spin polarization measured in the Tedrow-Meservey experiment [39] was established by Jullière [40]. Jullière's model for TMR is based on two assumptions. First, it assumes that spin of electrons is conserved in the tunneling process. If the two ferromagnetic films are magnetized parallel, the minority spins tunnel to the minority states and the majority spins tunnel to the majority states (Fig. 3, top panel). If, however, the two films are magnetized antiparallel the identity of the majority- and minority-spin electrons is reversed so that the majority-spins of the left electrode tunnel to the minority-spin states in the right electrode and vice versa (Fig. 3). Second, Jullière's model assumes that the conductance for a particular spin orientation is proportional to the product of the DOS of the two ferromagnetic electrodes. According to these assumptions, the conductance for the parallel and antiparallel alignment, $G_{P}$ and $G_{A P}$ respectively, can be written as follows:

$$
\begin{aligned}
& G_{P} \propto N_{L}^{\uparrow} N_{R}^{\uparrow}+N_{L}^{\downarrow} N_{R}^{\downarrow} \\
& G_{A P} \propto N_{L}^{\uparrow} N_{R}^{\downarrow}+N_{L}^{\downarrow} N_{R}^{\downarrow}
\end{aligned}
$$

where $N_{n}^{\uparrow}$ and $N_{n}^{\downarrow}$ are the DOS of the left and right ferromagnetic electrodes (designated by index $n=L, R$ ) for the majority- and minority-spin electrons. Substituting Eq. (15) to Eq. (14) we arrive at Jullière's formula 
$\mathrm{TMR}=\frac{2 P_{L} P_{R}}{1-P_{L} P_{R}}$

where $P_{L}$ and $P_{R}$ are the spin polarizations of the ferromagnetic electrodes given by Eq. (5).

From the discussion of Sec. 2.2 we know, however, that the spin polarization of the DOS alone is not relevant to describe tunneling experiments. Nevertheless, Jullière's model makes good sense because it disregards the nature of the DOS entering Eq. (5). As long as the conductance per spin can be represented as a product of two quantities characterizing left and right electrodes (or left and right interfaces), we arrive at Eq. (16) for TMR. A more realistic description of the tunneling conductance given by expression (13) assumes such a decoupling of the transmission coefficient and consequently allows one to represent TMR in form (13) with spin polarization given by Eq. (11). This makes the applicability of Jullière's formula more general.

Indeed it appears that the most recent spin polarization values with $\mathrm{Al}_{2} \mathrm{O}_{3}$ barriers, obtained using the Tedrow-Meservey technique, agree reasonably well with the maximum TMR values reported with $\mathrm{Al}_{2} \mathrm{O}_{3}$ barriers within Jullière's model (see Ref. 71 for details). Therefore it has become customary to interpret experimental data in terms of the Jullière formula, although this is an oversimplification of the factors that contribute to TMR. However, it is now commonly accepted that the spin polarization entering the Jullière formula is due to the ferromagnet/barrier complex rather than the ferromagnet alone.

\subsection{Electroresistance}

Yet another concept is the ferroelectric tunnel junction (FTJ), which takes advantage of a ferroelectric as the barrier material [78]. Ferroelectrics possess a spontaneous electric polarization that can be switched by an applied electric field. If the two interfaces are different a reversal of the barrier polarization orientation by external electric field can produce a large change in the electrical resistance of the FTJ, which is dubbed a giant electroresistance effect (GER) [79]. GER can be defined by
$\mathrm{GER}=\frac{G_{\rightarrow}-G_{\leftarrow}}{G_{\rightarrow}+G_{\leftarrow}}$,

where $G_{\rightarrow}\left(G_{\leftarrow}\right)$ are the conductances measured for the electric polarization of the barrier pointing towards the left (right) electrode respectively. Polarization affects the interface transmission function by changing (i) the electrostatic potential at the interface; (ii) interface bonding strength; and/or (iii) strain associated with the piezoelectric response (see Fig. 4).

In FTJs with a thin ferroelectric layer, the screening of polarization charges is incomplete and consequently there is a non-vanishing depolarizing field inside the ferroelectric. For an FTJ with asymmetric electrodes, e.g. having different density of carriers and screening lengths, the electrostatic potential associated with the depolarizing field is different depending on the direction of the electric polarization (see Sec. 5.1 for further discussion). This leads to the GER effect [79].

In addition, the reversal of the electric polarization in FTJs changes the direction of atomic displacements in the ferroelectric, thereby changing the atomic and electronic structure at the ferromagnet/barrier interfaces. Thus, in a junction with asymmetric interfaces this difference can translate into change of the junction resistance.

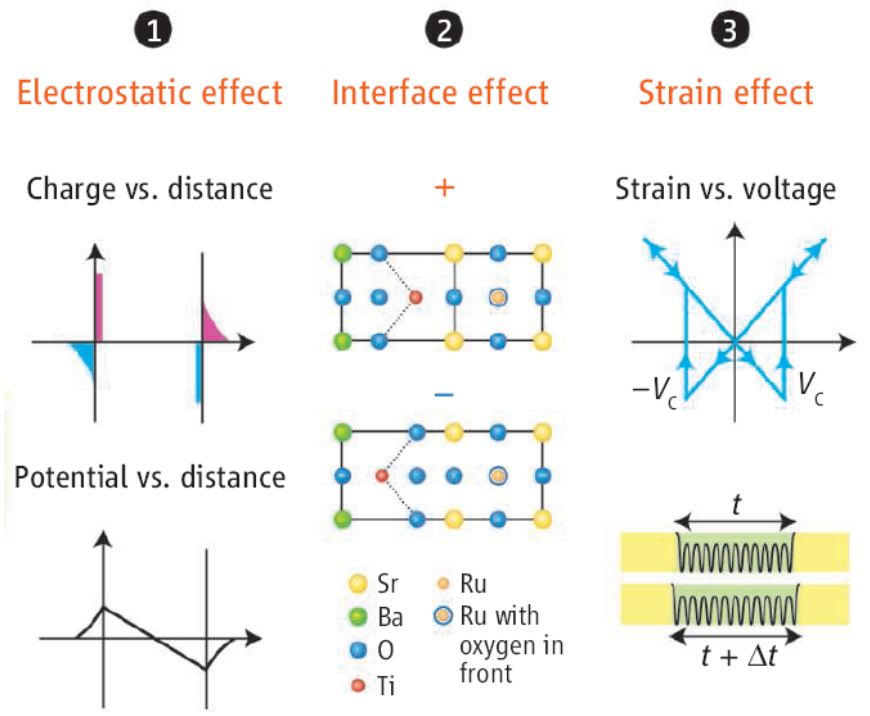

Fig. 4: Mechanisms affecting tunneling in ferroelectric tunnel junctions: (1) electrostatic potential at the interface, (2) interface bonding, (3) strain. Adopted from Ref. 78. 
Finally, ferroelectricity is produced by ionic displacements, thus it is intimately related to structural deformations and piezoelecticity. This, latter application of electric field (bias) can cause a physical deformation of the barrier affecting the barrier thickness, the structure of the interface, and the atomic displacements within the barrier. In addition the in-plane strain can influence the magnetization and the anisotropy of the electrodes (see Sec. 5).

\subsection{Band structure and symmetry matching}

Theoretical calculations of the ballistic conductance provide insights into the phenomenon of spin-dependent tunneling and magnetoresistance. Despite the fact that such calculations have become increasingly commonplace there is still considerable lack of intuition of what they entail. The key point to understanding ballistic conductance is that when the electron mean free path is comparable to the heterostructure dimensions the only source of resistance is reflection at the heterostructure interfaces. Thus, the conductance problem is boiled down to calculating reflection and transmission coefficients through a scattering region (barrier) attached to semi-infinite electodes. In the linear response regime (at small bias) only the electrons at the Fermi level participate in the transport. This problem is a generalized version of the tunneling through a rectangular potential barrier problem seen in elementary quantum mechanics.

We can carry the analogy between (i) the rectangular barrier problem and (ii) the general twoprobe setup deeper. First consider the electrodes: for (i) the solutions outside of the barrier are plane waves which are solutions of the Schrödinger equation with constant potential $V<E_{F}$. In (ii) the solid electrode solutions are propagating (Bloch) waves which are solutions of the Schrödinger equation for a periodic potential. There can be more than one such state corresponding to different bands crossing the Fermi level. These propagating states are found by solving the real band structure of the electrode material.

In the barrier region for (i) the solutions are decaying exponents corresponding to a solution of the Schrödinger equation for a constant potential $V>E_{F}$. For (ii) in the scattering region we have decaying (evanescent) wave functions corresponding to solutions of the Schrödinger equation for energies in the gap of the solid. It should be recognized that there is more than just one decay rate corresponding to tunneling through different higher laying bands. This is refered to as the complex band structure of the solid. Thus, the scattering region can be represented as a set of barriers which can be seen by the incoming electron depending on the symmetry of its wave function and its wave vector $\mathbf{k}_{\|}$.

Finally, in (i) the plane wave are matched to the decaying exponent (1-to-1) at the two interfaces to obtain the transmission coefficient. Similarly in (ii) the Bloch states in the electrodes are required to match the evanescent states in the barrier (many-to-many) at both interfaces to obtain transmission coefficients for each state on the one side to each state on the other side.

\subsubsection{Complex band structure}

By band structure we mean the available energies for a given k point, $E=E(\mathbf{k})$, obtained as the solution of the Schrödinger equation $(H-E) \psi=0$. The wave function is expanded in a set of atomic orbitals $\psi_{\mathbf{k}}(\mathbf{r})=\sum_{\mathbf{R}, \alpha} e^{i \mathbf{k} \cdot \mathbf{R}} C_{\alpha}|\mathbf{R}, \alpha\rangle$, where $\mathbf{R}$ are the atomic positions and $\alpha$ is a combined orbial/spin index. Then the Schrödinger equation is solved for its eigenvalues (energy bands) and eigenvectors (wave function coefficients).

In a layer geometry $\mathbf{k}=\left(\mathbf{k}_{\|}, k_{\perp}\right)$ and the wave function expansion, $\psi_{\mathbf{k}_{\|}, k_{\perp}}(\mathbf{r})=\sum_{l, \alpha} e^{i k_{\perp} l} C_{\alpha}\left|k_{\|}, l, \alpha\right\rangle$, where $l$ denotes the layer positions and $\left|k_{\|}, l \alpha\right\rangle=\sum_{\mathbf{R}_{\|}} e^{i \mathbf{k}_{\|} \cdot \mathbf{R}_{\|}}\left|\mathbf{R}_{\|}, l \alpha\right\rangle$ are called planar orbitals because they are centered at a given layer [114]. Then, the band structure is determined by the eigenvalues of the equation

$$
\sum_{j} C_{j} e^{i k_{\perp} j}\left(H_{i j}-E S_{i j}\right)=0
$$

where $H_{i, j}=\left\langle\mathbf{k}_{\|}, i|H| \mathbf{k}_{\|}, j\right\rangle$ is the Hamiltonian matrix element and $S_{i, j}=\left\langle\mathbf{k}_{\|}, i \mid \mathbf{k}_{\|}, j\right\rangle$ is the planar orbital overlap. This problem is usually solved in reverse, i.e., for given $E$ and $\mathbf{k}_{\|}$all the possible values of $k_{\perp}$ are found by doubling the size of the linear system of equations [115]. In this way all formal 
solutions (both real and complex) are obtained. We can find all propagating $\left(k_{\perp} \in \mathbb{R}\right)$ and evanescent $\left(k_{\perp} \in \mathbb{C}\right)$ states for the bulk materials on both sides of the interface. The eigenvalues come in pairs: propagating $\left(v_{g}>0\right.$ if $\left.k_{\perp} \in \mathbb{R}\right)$ or decaying to the right $\left(\operatorname{Im} k_{\perp}>0\right.$ if $k_{\perp} \in \mathbb{C}$ ) or and propagating $\left(v_{g}<0\right.$ if $\left.k_{\perp} \in \mathbb{R}\right)$ or decaying $\left(\operatorname{Im} k_{\perp}<0\right.$ if $\left.k_{\perp} \in \mathbb{C}\right)$ to the left, where $v_{g}$ is the group velocity. The evanescent states connect to the propagating states only in critical points, such as zone edges or band crossings. Therefore, only propagating and evanescent states of the same symmetry have non-vanishing matrix elements [116].

The collection of all propagating and evanescent states in a given range of energies is known as the complex band structure of the solid. In systems with interfaces, complex solutions of $k_{\perp}$ which decay away from the interface are legitimate solutions. In bulk materials complex values of $k_{\perp}$ are not normalizable at infinity and the band structure is completely real. However, even in this case complex solutions are usefull because they provide the decay rates for carriers injected at energies falling in the gap of insulators or semiconductors. Finally, we do need all real and complex solutions in order to set up correctly the scattering problem.

\subsubsection{Interface matching: reflection and transmission coefficients}

In order to obtain reflection and transmission coefficients we first consider the simplest case of one interface between the two materials $I$ and $I I$ (Fig. a). We investigate the matching at the boundary of the states on the left to the states on the right. There is one incoming state in region $I, k_{>}^{I, i}$, one of the right going propagating states in this material. This state is partially reflected at the interface in a number of left going states $k_{<}^{I, j}$ in region $I$ and partially transmitted to right going states $k_{>}^{I I, j}$ in region II. The wave function in the presence of the interface in response to an incoming state from the left is

$$
\begin{aligned}
\psi_{i} & =\sum_{l=-\infty}^{0} C_{l}^{I, i} e^{i k_{>}^{I, i} l}\left|\mathbf{k}_{\|}, l\right\rangle^{I}+\sum_{j} r_{i j} \sum_{l=-\infty}^{0} C_{l}^{I, j} e^{i k_{\llcorner}^{I, j} l}\left|\mathbf{k}_{\|}, l\right\rangle^{I} \\
& +\sum_{j} t_{i j} \sum_{l=1}^{\infty} C_{l}^{I I, j} e^{i k_{\llcorner}^{I I, j} l}\left|\mathbf{k}_{\|}, l\right\rangle^{I I}
\end{aligned}
$$

where it is assumed that the interface separates layers $l=0$ (material $I$ ) and layer $l=1$ (material $I I)$, and the three terms stand for incoming wave, reflected wave, and transmitted wave respectively. The index $i$ labels one of the right-moving propagating states in region $I$ and the sum over $j$ runs through all (propagating and evanescent) left going states in region $I$ in the first term and all right going states in region $I I$ in the last term. Although evanescent states will not contribute to the current both propagating and evanescent states have to be included in order to satisfy the boundary conditions.

The Schrödinger equation in the presence of the interface is $\left\langle\mathbf{k}_{\|}, m|(H-E)| \psi_{i}\right\rangle=0, \quad$ where $m=-\infty, . ., \infty$ runs over all layers in the electrodes and the sample. If $m$ is far from the interface then each of the terms in the equation automatically satisfies the Schrödinger equation, Eq. 18, for that material and the above equation will be satisfied trivially. Only a finite number of equations around the interface will be nontrivial which could be solved for the unknown coefficients [117].

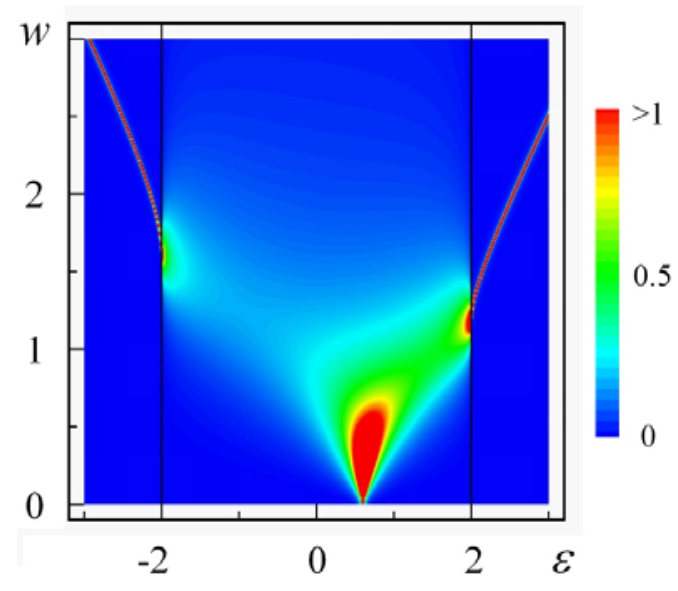

Fig. 5: Formation of interface resonant states from pure interface states within a one-dimensional tight-binding model representing a semi-infinite metal electrode connected to an insulator. DOS on the interface atom is shown as a function of the reduced energy $\varepsilon$ and the bond strength $w$ between the interface atom and the electrode. A one dimensional chain forms a continuum of states, which expand from $\varepsilon=-2$ to $\varepsilon=$ 
2. When the bonding is strong the bonding-antibonding states lie beyond the continium, and therefore represent pure interface states. When the bonding becomes weaker the bonding-antibonding states fall in the continuum and develop into interface resonances. Adopted from [118].

This set of equations allows us to obtain the transmission (matching) $t$ coefficients between every pair of states at the interface. We can distinguish several possible scenarios: first, if $E$ falls in the real bands of both materials, there will be wave propagation through the interface corresponding to nonzero matching coefficients between propagating states in $I$ and II. If the states on both sides are similar then $t \approx 1$ and if they are dissimilar then $t \ll 1$. Moreover, a propagating state can transmit in more than one state on the other side.

Second, if $E$ falls in a gap of one of the materials, there will be nonzero matching coefficients between propagating states on the one side and evanescent states on the other side, i.e., metal-induced gap states. These play a major role in determining the position of the Fermi level and band alignment.

Finally, if $E$ falls in a gap of both materials, there are nonzero matching coefficients between evanescent states on both sides, which gives rise to pure interface states. Since in this case there is no incoming wave, the system of equations is homogeneous and the determinant of the coefficients must vanish, which is a criterion for the existence of pure interface states. In metals pure interface states may exist in parts of the Brillouin zone with no real bands. Where real bands are present the interface states hybridize with the bulk states and develop into interface resonances. Fig. 5 illustrates the formation of interface resonant states within a one-dimensional tight-binding model $[118,119]$.

\subsubsection{Relation to transport}

Similar formalism can be invoked to relate the wavefunction matching to the transport through layered heterostructures such as spin valves, MTJs, and FTJs [120]. In this case, as is seen in Fig. 2b, there is a finite size slab (region III) between the two semi-infinite electrodes (regions $I$ and $I I$ ). Again we consider an incoming state from the left electrode $k_{>}^{I, i}$ which is partially reflected at the interface in a number of left going states $k_{<}^{I, j}$ in region $I$ and partially transmitted to right going states $k_{>}^{I I, j}$ in region II. However, these states are not directly matched to each other but instead they are independently matched to the wave function in the slab at each interface. The slab wave function is written as a linear combination of all possible solutions in region III. The wavefunction of the whole system can be written as

$$
\begin{aligned}
\psi_{i} & =\sum_{l=-\infty}^{0} C_{l}^{I, i} e^{i k{ }_{>}^{I, i} l}\left|\mathbf{k}_{\|}, l\right\rangle^{I}+\sum_{j} r_{i j} \sum_{l=-\infty}^{0} C_{l}^{I, j} e^{i k_{\llcorner}^{I, j} l}\left|\mathbf{k}_{\|}, l\right\rangle^{I}+ \\
& +\sum_{l=1}^{N} \sum_{j}\left(a_{i j} C_{l}^{I I I, j} e^{i k_{\llcorner}^{I I I, j} l}+b_{i j} C_{l}^{I I I, j} e^{i k_{\supset}^{I I, j},}\right)\left|\mathbf{k}_{\|}, l\right\rangle^{I I I}+ \\
& +\sum_{j} t_{i j} \sum_{l=N+1}^{\infty} C_{l}^{I I, j} e^{i k_{>}^{I I, j} l}\left|\mathbf{k}_{\|}, l\right\rangle^{I I}
\end{aligned}
$$

where the third term contains a linear combination of all (propagating and evanescent) states within the slab material [117]. Again the solution of the Schrödinger equation $\left\langle\mathbf{k}_{\|}, m|(H-E)| \psi_{i}\right\rangle=0$ leads to expressions for the transmission amplitudes $t$ between states on the left and on the right of the spacer. In terms of these, the transmission is given by

$T=\operatorname{Tr}\left[t t^{\dagger}\right]$.

This allows for calculation of the conductance using the Landauer-Büttiker expression (2) (for recent review see Ref. 121). Although evanescent states are necessary for a proper accounting of the boundary conditions at the interfaces, far from the interfaces only the propagating states contribute to the transport.

The relationship between wave function matching at the interfaces and the conductance sheds more light on the origin of TMR. Spin-dependence of the tunneling current can be deduced from the symmetry of the Bloch states in the bulk ferromagnetic electrodes and the complex band structure of the insulator $[67,122]$. The first observation is that electrons tunneling with $\mathbf{k}_{\|} \neq 0$ travel further in the insulator; therefore, if the insulator is thick only states around the $\bar{\Gamma}$ point $\left(\mathbf{k}_{\|}=0\right)$ contribute to the conductance. States at the $\bar{\Gamma}$ point have a well defined symmetry. By identifying those 
bands in the electrodes that are coupled efficiently to the evanescent states decaying most slowly in the barrier, one can draw conclusions about the spin polarization of the conductance.

For a broad class of insulating materials the states belonging to the identity representation (i.e. those that are fully symmetric) should have minimum decay rates. This representation is comparable to the $s$ character suggesting that $s$ bands should be able to couple most efficiently across the interface and decay slowest in the barrier. For $\mathrm{Fe}$, Co, and $\mathrm{Ni}$ ferromagnets the majorityspin states at the Fermi energy have more $s$ character than the minority-spin states. Thus, the majority conductance is expected to be greater than the minority conductance resulting in a slower decay with barrier thickness for the former.

These symmetry arguments are illustrated in Fig. 6 for $\mathrm{Fe}$ and $\mathrm{MgO}$ and explain nicely the large values of TMR predicted for epitaxial $\mathrm{Fe} / \mathrm{MgO} / \mathrm{Fe}$ junctions $[67,68]$. Away from the $\bar{\Gamma}$ point the states are superpositions of the different symmetries and they exhibit different rates of decay in the barrier. First the decay rate is high as the faster decaying components die out, and then all states decay with the same decay rate. Thus, if a larger portion of the Brillouin zone contributes to the conductance, TMR decreases.
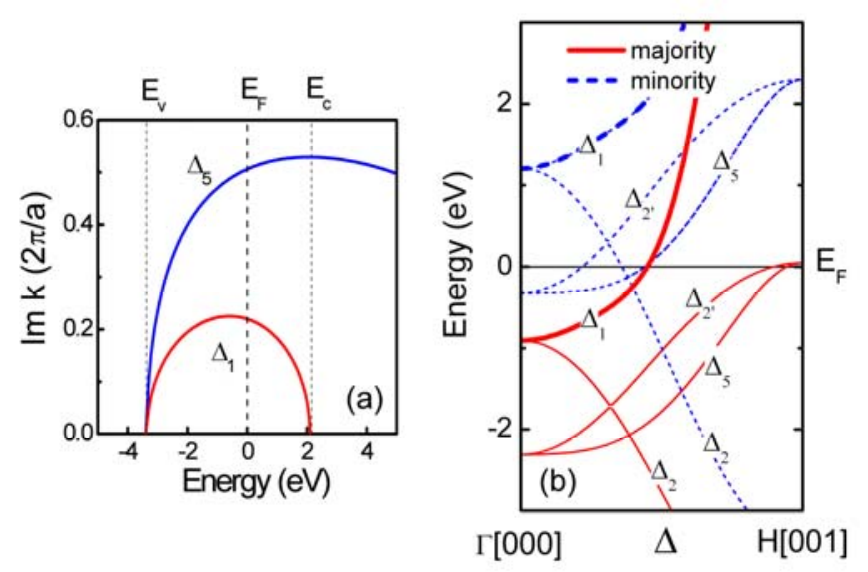

Fig. 6: (a) Complex band structure of $\mathrm{MgO}$ at the $\bar{\Gamma}$ point. Two evanescent states with lowest decay rates are shown. The position of the Fermi level, $\mathrm{E}_{\mathrm{F}}$, in a $\mathrm{Fe} / \mathrm{MgO} / \mathrm{Fe} \mathrm{MTJ}$, the top of the valence band, $E_{v}$, and the bottom of the conduction band, $\mathrm{E}_{\mathrm{c}}$, are displayed by dashed lines. (b) Spin-resolved band structure of $\mathrm{Fe}$ along the [001] direction. Bands are labeled by their symmetry representation. Thick lines mark the $\Delta_{1}$ bands that match the symmetry of the evanescent state in $\mathrm{MgO}$ having the lowest decay rate. Taken from Ref. 72 .
Although for $s p$-bonded insulators the $s$-like states have the lowest rate of decay there are some cases like $\mathrm{TiO}_{2}, \mathrm{SrTiO}_{3}$, and other transition metal oxides in which the complex band structure of the insulator has multiple evanescent states with comparable rates of decay that allow efficient tunneling of the $d$ states from the electrodes [123]. Negative spin polarization was observed for the $\mathrm{Co} / \mathrm{SrTiO}_{3}$ [124], $\mathrm{Co} / \mathrm{TiO}_{2}$ [125] and $\mathrm{Co} / \mathrm{LaAlO}_{3}$ interfaces [126]. For the former two interfaces the negative spin polarization is due to the $\mathrm{Ti}$ $3 d$ states which have a large density in the minority-spin channel at the Fermi energy, whereas for the latter interface it is probably due to tunneling through the La $4 d$ states [127].

In general, if there is one state in the barrier of an MTJ with much lower rate of decay than the rest, conductance is proportional to $T_{\mathbf{M}_{L}}^{L} e^{-2 \kappa d} T_{\mathbf{M}_{R}}^{R}$, where $T_{\mathbf{M}}$ is the interface transmission function for a given direction of the magnetization in the electrodes, $\mathbf{M}$. Thus, substantial imbalance between majority and minority conductance occurs when there is a state in one of the spin channels which matches the evanescent state at the interfaces much better than any of the states in the other channel, e.g. $T_{\uparrow} \gg T_{\downarrow}$ as in the case of $\mathrm{Fe} / \mathrm{MgO}$ interface. Thus, $G_{\uparrow \uparrow} \gg G_{\downarrow \downarrow}$ which produces the large spin polarization. Also, $G_{P}=G_{\uparrow \uparrow}+G_{\downarrow \downarrow}$ $\gg G_{A P}=G_{\uparrow \downarrow}+G_{\uparrow \downarrow}$ which is the source of the large TMR ratio.

Similarly, in an FTJ the conductance is proportional to $T_{\mathbf{P}}^{L} e^{-2 \kappa d} T_{\mathbf{P}}^{R}$ where $T_{\mathbf{P}}$ is the interface transmission function for a given direction of the polarization of the barrier, $\mathbf{P}$. Let us assume one interface ferroelectrically dead, e.g. $T_{\mathbf{P}}^{R}=$ const as in the case of $\mathrm{BaO}$ terminated $\mathrm{BaTiO}_{3}$. Then, ferroelectric displacement of the interface atoms will cause imbalance between the two polarization states, $T_{\leftarrow} \neq T_{\rightarrow}$, which leads to $G_{\leftarrow} \neq G_{\rightarrow}$ and the GER effect.

Symmetry considerations also play an important role, in scanning tunneling microscopy (STM) as well. Evidence of coverage dependent band structure symmetry effects in STM has been identified for Gd overlayers $[128,129]$.

These symmetry arguments have their limitations. First, they assume that the barrier is sufficiently thick so that only a small focused region of the surface Brillouin zone contributes to the tunneling current. For realistic 
MTJs with barrier thickness of about $1 \mathrm{~nm}$ this assumption is usually unjustified. Second, despite the presence of certain selection rules for tunneling, there is no general rule preventing the Bloch states composed mostly of the $d$ orbitals from tunneling through barrier states that have no $d$ character. Symmetry strictly forbids tunneling only in special geometries for special values of the wave vector. Therefore, symmetry considerations alone are not always sufficient to predict the spin polarization. It is critical to take into account the electronic structure of the ferromagnet/barrier interfaces, which controls the spin polarization.

\subsection{Interface states}

It is well-known that metal surfaces often exhibit electronic bands that are localized at the surface [88,130-133]. A similar behavior occurs at metalinsulator interfaces, as well as semiconductor-insulator interfaces. The respective localized bands at the interface are known as interface states. In essence, the origin of all such states may be construed as lying in the breakage of translational symmetry inherent in creation of the surface or interface. Combinations of energy and wave vector that are disallowed in the bulk (due to the confluence of elemental composition and symmetry considerations) become permitted at the surface, although they must necessarily decay rapidly within the selvedge.
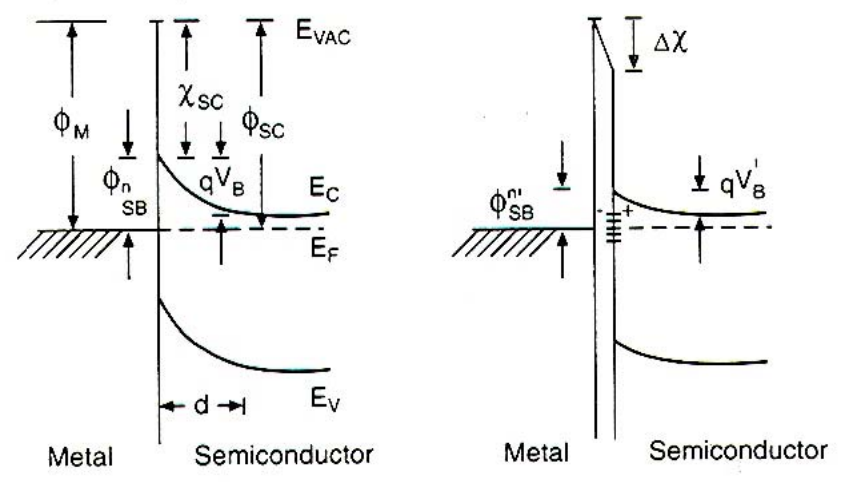

Fig. 7: Schematic energy band diagram of n-type semiconductor-metal contact for (a) abrupt junction with no interface states and (b) with interface states. Adopted from Ref. 91.

The topic of interface states has received a lot of attention in the context of the technologically important metal-semiconductor interfaces. In order to explain the rectifying behavior of such contacts, Schottky [134] and Mott [135] suggested the existence of a potential barrier at the interface (Schottky barrier). Quantitative predictions of the Schottky barrier height based on the Schottky-Mott model did not fit early experimental evidence $[89,90]$. This was attributed to the presence of interface states in the gap, including, intrinsic interface states [136], metal-induced gap states [137], as well as defect- or disorder-related states. These models assume that the distribution of states in the gap is essentially a semiconductor property and the charge neutrality level of the gap states determines the Schottky barrier height. Later, it was proposed that polarization of chemical bonds at the interface may account for the actual Schottky barrier height [138].

The Schottky-Mott model is a non-interacting model, which does not take into account charge redistribution across the interface. The second law of thermodynamics requires that the electrochemical potentials on both sides of the interface are the same at equilibrium, $\mu_{I}=\mu_{I I}$. For metal-metal interfaces, where the two metals have different work functions $\left(\varphi_{I}\right.$ and $\left.\varphi_{I I}\right)$ the alignment of the electrochemical potential causes a potential difference across the interface, $\Phi=\varphi_{I}-\varphi_{I I}$ [88]. The potential is screened by the electron gas within a few Ångstroms, which create an interface dipole layer several lattice parameters in size.

For metal-semiconductor interfaces, the SchottkyMott model predicts that Schottky barrier height equals the difference between the metal work function, $\varphi_{M}$, and the semiconductor majority carrier band edge (see Fig. 7a). For an $n$-type semiconductor

$\Phi_{n}=\varphi_{M}-\chi_{S}$

where $\chi_{s}$ is the electron affinity. For a $p$-type semiconductor

$\Phi_{p}=I_{S}-\varphi_{M}$

where $I_{S}$ is the ionization energy. This potential is also screened, but due to the low carrier concentration in the semiconductor it produces a macroscopic charge depletion region resulting in band bending. This noninteracting model predicts strong dependence of the Schottky barrier height on the metal work function which is not confirmed experimentally. 
The problem is addressed by taking into account the charge rearrangement at the interface. The barrier height becomes

$$
\Phi_{p}=I_{S}-\varphi_{M}-e \Delta
$$

where $\Delta$ is the potential drop at the interface due to this charge transfer producing an interface dipole. The interface dipole is essential for the band alignment at the interface and the overall transport properties. Various competing theories differ as to the origin of the charge transfer.

One possibility is the pinning of the Fermi energy by interface states as illustrated in Fig. 7b. Intrinsic interface states are states localized close to the interface, formed by matching the evanescent solutions of the Schrödinger equation on either side of the interface [136]. If these states fall in the gap of both materials they are pure interface states. Otherwise, they are mixed with propagating states and form interface resonances, which can propagate far into the insulator (semiconductor). Upon contact, the charge may be trapped by the interface states resulting in the pinning of the Fermi level. Defect- or disorder-induced interface states act in a similar way.

Second, there exist metal-induced gap states in an insulator (semiconductor). These are propagating states in the metal that penetrate across the metal/insulator (semiconductor) interface in the gap of the insulator (semiconductor) due to coupling to evanescent states [137]. The charge density in the gap is compensated by loss of charge density in the valence and conduction bands. There is a charge neutrality level (CNL), somewhere close to midgap, at which the valence and conduction band contributions to the density of states is equal. Uncompensated charge causes large electrostatic potential and, therefore, it is argued that the Fermi level lies close to the charge neutrality level. It can be shown that charging the gap states acts as a negative feedback to dampen changes in the metal work function

$\Phi_{p}=\gamma_{G S}\left(I_{S}-\varphi_{M}\right)+\left(1-\gamma_{G S}\right) \phi_{C N L}$.

Here $\phi_{C N L}$ is the potential corresponding to the charge neutrality level. $\gamma_{G S}=1$ if the density of the gap states $D_{G S}$ is zero (in which case we recover the SchottkyMott model) and $\gamma_{G S}=0$ if $D_{G S}$ is large [138].
Finally, chemical bond polarization is the redistribution of charge in the interface band when the two atoms have different electronegativity. In this case, the above expression (25) applies but the coefficient $\gamma_{B}$ is related to bond polarization [138]. Although it is certain that all these processes take place at the interface, it is very difficult to assess experimentally their relative importance.

The same reasoning applies to the problem of band alignment in metal-insulator interfaces. The main difference is that there are no free carriers in the insulator so there is no screening of the interface dipole and no band bending. In general, the size of the depletion region in semiconductors is of the order of microns, which implies that band bending will not be an important effect in nm-scale heterojunctions.

\subsection{Resonant transmission}

The presence of localized states in the tunnel heterostructures can significantly modify the mechanism of tunneling, leading to so-called resonant transmission. There are two distinct types of resonance phenomena associated with localized states at the interfaces of the heterostructure (due to interface states) [139] or in the barrier layer (due to defects or impurities) [140].

Interface states in MTJs appear as states with high density of states close to the interface and decaying exponentially away from the interface. Their natural width, $\Gamma$, is related to the coupling of the interface states to bulk states in the electrodes through various scattering mechanisms. Due to the localization, interface resonances have very large DOS in a certain narrow energy range close to the Fermi energy due to interface state pinning of the Fermi level. In asymmetric junctions this increased DOS translates to higher transmission due to tunneling through the interface resonance. In symmetric junctions, however, two interface resonances can form bonding and antibonding states in a manner analogous to bonding in diatomic molecules [139]. If the lifetime of the interface resonances is large enough and the barrier thickness small enough so bonding and antibonding states can form then both electrons reside in the symmetric (bonding) state and traverse the barrier coherently i.e. without tunneling and respectively attenuation. This type of resonant transmission has been shown to be very important for very thin MTJs (see Sec. 3.3). 
A different type of resonant effect appears when there are localized states inside the barrier due to defects. In this case the electron can experience enhanced transmission due to hopping through the defect level if their energies coincide. The prototype system to study resonant tunneling is a quantum well between two tunneling barriers [97]. If a quantum well level falls between the chemical potentials of the electrodes, the transmission in the vicinity of the level is

$T_{\sigma}=\frac{\Gamma_{L \sigma} \Gamma_{R \sigma}}{\left(\varepsilon-\varepsilon_{d}\right)^{2}+\left(\Gamma_{L \sigma}+\Gamma_{R \sigma}\right)^{2} / 4}$,

where $\Gamma_{L(R)}$ is the level broadening due to the left (right) electrode, $\varepsilon_{d}$ is the defect level position, and $\sigma$ indicates the spin channel. The transmission has a resonance at energy $\varepsilon=\varepsilon_{d}$. This type of resonant transmission can be important for very thin barriers in which transport properties can be determined by the presence of a single defect (see Sec. 4.4).

\section{Interface electronic structure}

It might be argued that the influence of surface- or interface-localized states on electronic transport properties would be minimal, because they do not extend into the bulk of the metal and thus cannot support a current. Through their coupling with propagating states, however, it is evident that such localized states can, in fact, contribute significantly to the overall flow of current through a surface or interface. There are a number of scattering mechanisms contributing to the coupling: phonon, magnon and scattering from defects.

Typical phonon energies are very small compared to the Fermi energy, but they do carry significant crystal momentum. Their emission or absorption can allow scattering of electrons and holes between surface states near the zone center and bulk states nearer the zone edge. In this way, current may flow into or out of the surface by means of a two-step process: tunneling between tip and surface state (in the case of STM), or across an interface; followed by scattering between the surface/interface states and bulk states. Since surface states extend considerably further into the vacuum than typical bulk states, and interface states may extend deep into a dielectric in a tunnel junction, this scattering- assisted process can be competitive with direct tunneling via bulk-like states, provided that the scattering rate is not too slow.

The case of magnon scattering is rather analogous to phonon scattering, in that it produces a large change in crystal-momentum and a small change in energy, but with the added characteristic of reversing the electron spin. Similarly, the symmetry-breaking associated with defect scattering allows a relaxation of crystalmomentum conservation, just as in a non-magnetic system. Now, however, the spin of the scattered electron or hole may be exchanged with that of an electron or hole localized in the incomplete shell of the defect site. Either way, electrons or holes in the minority-spin surface states may be transferred to majority-spin bulk states and vice versa. Since the surface states again extend into the vacuum rather further than do the bulk states, scattering-assisted transport across the boundary is likely to be competitive with direct tunneling via majority-spin states.

It was found that surface resonant states may produce a strong angular dependence of the conductance when the saturation magnetization of the entire junction changes its direction [141-143]. This phenomenon is known as tunneling anisotropic magnetoresistance (TAMR). It was noticed that the spin-flip scattering associated with the resonant states strongly depends on the intrinsic broadening of this states [141].

\subsection{Non-oxide interfaces}

The classic example of the role of non-magnetic surface states in STM is intended to underline the importance of scattering in allowing localized states to play a major role in mediating current flow across an interface. Certain metals have bulk band structures which display a partial gap at the Fermi level when projected onto the surface Brillouin zone. Such a gap can support Shockley-type surface states, whose parabolic dispersion curves betray two-dimensional free-electron-like behavior (see Fig. 8). Clearly, these states are quite capable of supporting a current parallel to the surface, but not in the direction normal to the surface. Nevertheless, STM experiments on $\mathrm{Cu}(111)$ [144-147], $\operatorname{Ag}(111)$ [148,149], $\mathrm{Au}(111)$ [147- 153] and a range of other surfaces [154-158] have revealed that the tunneling current under certain tip and bias conditions may actually be entirely dominated by surface state effects. It is important to realize that surface states are not completely decoupled from the 
bulk of the semi-infinite crystal. Although the location of these two-dimensional bands in a bulk-forbidden gap suppresses hybridization with three-dimensional bands, electrons and holes may nevertheless be transferred from one to the other through scattering processes, subject to the twin constraints of energy and crystalmomentum conservation (as discussed above).

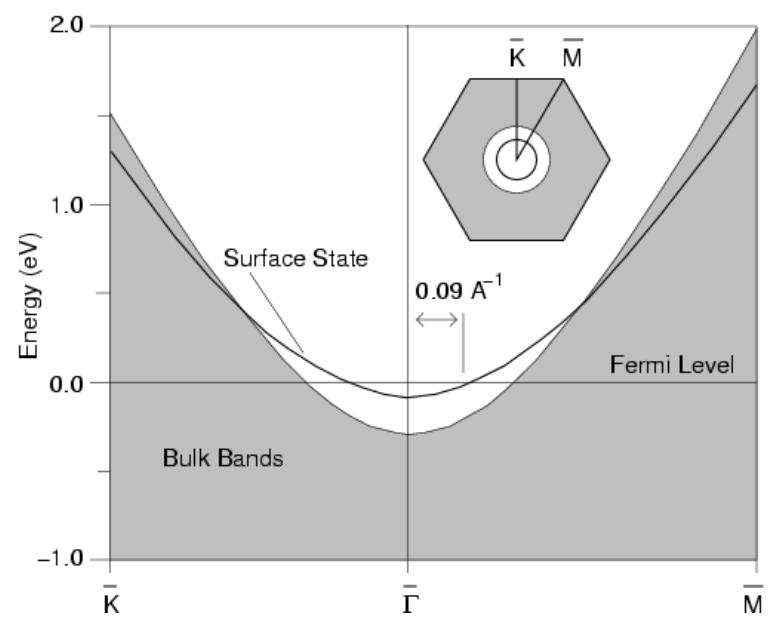

Fig. 8: Projected bulk band structure and surface state of $\operatorname{Ag}(111)$, with the surface Brillouin zone shown inset, from a density functional calculation by Jenkins [159].

There are at least two non-oxide dielectric materials for which the magnetic interface states (arising from the ferromagnet surface states) have been identified experimentally. The low $\mathrm{Z}$ inorganic boron carbide $\mathrm{B}_{5} \mathrm{C}$ can be grown by chemical vapor deposition without pinholes in the ultrathin film regime. The band gap of boron-carbide $\left(\mathrm{a}-\mathrm{B}_{x} \mathrm{C}: \mathrm{H}\right)$ can be adjusted from $0.7 \mathrm{eV}$ to $1.9 \mathrm{eV}$ by altering the boron to carbon ratio [160] and well above $2.7 \mathrm{eV}$ by adding phosphorus to the alloy [161]. Magnetic tunnel junctions based on boron carbide have been made [162], and the results suggest that the magnetic interface states are not completely suppressed with this dielectric barrier layer.

Similarly, experimental band mapping [163,164] has provided evidence of changes in the spin-dependent Fermi surface when h-BN overlayers are deposited on Ni(111), but the magnetic surface states appear to be enhanced and not suppressed by the overlayer. This suggests that the boron based barrier layers may be less likely than many other materials to suppress the interface magnetization: key to a good tunnel junction $[105,165]$. Indeed, a magneto-resistive device has been fabricated using boron nitride as the dielectric (insulating) barrier layer [166], which may also be grown by chemical vapor deposition.

\subsection{Half-metallic interfaces}

An extreme example of the importance of interface states occurs in the ground state of nominally halfmetallic systems. Half metals are materials in which one spin band has a gap around the Fermi energy [74]. Many compounds have been predicted to be half metallic, such as the half- and full-Heusler alloys $\mathrm{NiMnSb}$ [74] and $\mathrm{Co}_{2} \mathrm{MnSi}$ [167]; the oxides $\mathrm{CrO}_{2}$ [168], $\mathrm{Fe}_{3} \mathrm{O}_{4}[169,170]$, and $\mathrm{La}_{0.67} \mathrm{Sr}_{0.33} \mathrm{MnO}_{3}$ [171, 172]; and the sulfide $\mathrm{Co}_{x} \mathrm{Fe}_{1-x} \mathrm{~S}_{2}$ [173]. Only a few materials like $\mathrm{La}_{2 / 3} \mathrm{Sr}_{1 / 3} \mathrm{MnO}_{3} \quad[174,175]$, $\mathrm{NiMnSb}$ [176,177], and $\mathrm{CrO}_{2}$ [178-180,181,182] have direct experimental evidence suggesting the high polarizations typical of postulated half-metallic behavior, but always with limited wave vector sampling in the case of spinpolarized photoemission and spin-polarized inverse photoemission measurements. Other measurements indicating very high polarization are also fraught with difficulties in their interpretation [76,167,185]. In general, the experimental evidence in favor of halfmetallic behavior is not conclusive [76,159,183-187]. Among the many reasons for less than $100 \%$ polarization are correlation effects [188-192], magnons [147,183-187,193,194], and irreversible interface compositional changes. Half-metallicity also quickly deteriorates with temperature as the importance of these factors increases.

There are also the problems associated with surface and interfaces states. Minority spin surface states in the half-metallic systems are well known [195- 201] and can 'develop' into interface states in the presence of overlayers [198-201]. The creation of more interfaces increases the minority-spin population with the consequent loss of half-metallic character. 

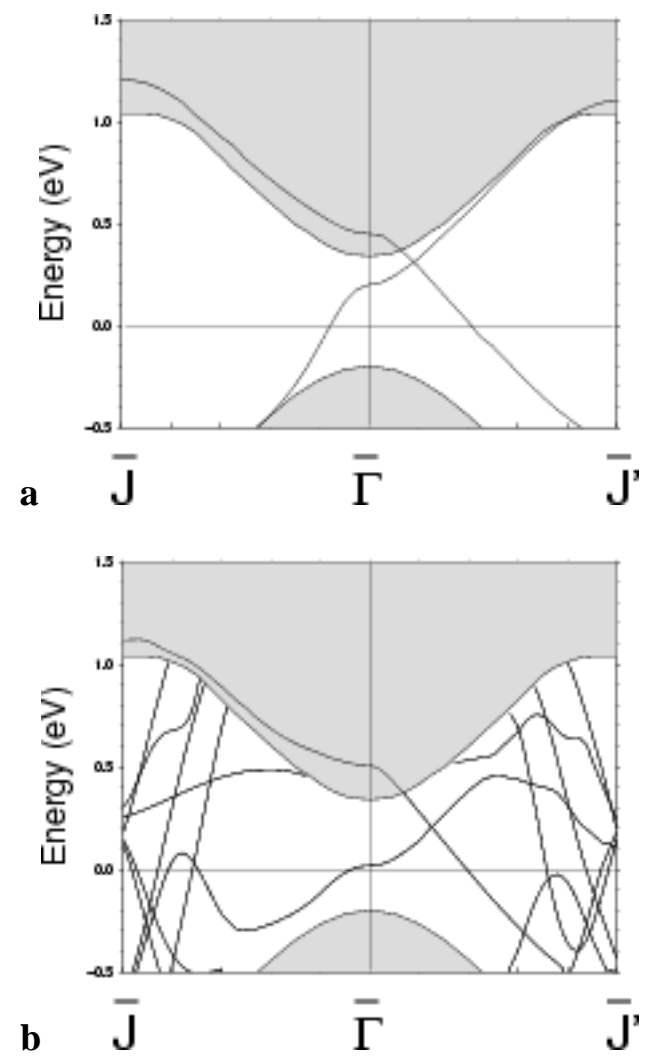

Fig. 9: Projected bulk minority-spin band structures of $\mathrm{NiMnSb}$ (shaded) showing (a) DFT-calculated surface states of $\mathrm{NiMnSb}(001)$, and (b) interface and overlayer states of $\mathrm{NiMnSb}(001) / \mathrm{Sb}$, adapted from $[202,199]$.

Consider the (001) surface of NiMnSb (whose electronic band structure is illustrated in Fig. 9a). The electronic structure of the surface region is not found to be half-metallic: minority-spin surface states encroach into the band gap region, crossing the Fermi level along an elliptical contour in reciprocal space [199]. The tunneling via the localized minority-spin surface states at the Fermi level can contribute to the overall current. There is no bulk-like minority-spin state near the Fermi level, even at the zone edge in this system. However, as was discussed above, the interface states can be populated through electron-electron, electron-phonon, and electron-magnon scattering, resulting in a sizeable contribution to the tunneling current [203].

In principle, it may at least be possible to ameliorate some of the worst effects of the interface states by engineering the electronic structure of the interface. As stressed above, crystal momentum is a conserved quantity in transport across an ideal surface or interface, so that a ballistic device, in which current flows primarily through states of zero parallel crystal momentum, might not be too badly affected so long as the interface states are avoided at the zone center at the energy of choice. This raises the issue of engineering the detailed electronic structure through appropriate manipulation of atomic structure.

The nature of $\mathrm{NiMnSb}(001)$ surface states is, indeed, highly unusual, resembling neither Shockley nor Tamm typology: the dispersion curves are parabolic, but the effective mass suffers an apparent change in sign when the surface wave vector is rotated by $90^{\circ}$ (see Fig. 9a). The effect seems to be due to the interplay between the $\mathrm{Sb}$ p-orbital and $\mathrm{Mn}$ d-orbital contributions to the surface states, and the fact that these are clearly modulated by the arrangement of second-layer Ni atoms. The result is a (001) surface that has $\mathrm{C}_{2 \mathrm{v}}$ symmetry and the allowed irreducible representations are $A_{1}\left(s, p_{z}, d_{3 z^{2}-r^{2}}, d_{x} 2+y 2\right), A_{2}\left(d_{x y}\right), B_{1}$ $\left(\mathrm{p}_{\mathrm{x}}, \mathrm{d}_{\mathrm{xz}}\right)$ and $\mathrm{B}_{2}\left(\mathrm{p}_{\mathrm{y}}, \mathrm{d}_{\mathrm{yz}}\right)$.

In fact, the energy-wave vector surfaces mapped out by these states may be visualized as two sheets, touching at just two points (electronic conical intersections) in the Brillouin zone. The lower sheet displays $A_{1}$ symmetry character at the zone center, while the upper displays $\mathrm{B}_{1}$ symmetry at the same point; the Fermi contour varies between $\mathrm{A}_{1}$ symmetry at its greatest extent along the $\overline{\Gamma \mathrm{J}}$ direction, and $\mathrm{B}_{1}$ symmetry at its greatest extent along the $\overline{\Gamma J^{\prime}}$ direction. The consequences of such unusual dispersion for spininjection can, at present, only be guessed. What seems apparent is that minority-spin electrons injected into the upper surface state would be somewhat constrained (in the absence of spin-flip scattering and radiative transitions) to follow a very particular path through one or other of the two conical intersections. More usefully, perhaps, we might note that majority-spin electrons injected at the zone center just above the Fermi level (or majority-spin holes injected just below) would be relatively immune to spin-flip scattering, due to the absence of minority-spin surface states from that region (although roughness-induced non-conservation of parallel momentum, or interaction with a magnon of finite wave vector could still contribute). A small window is thus apparent, within which strongly spinpolarized conduction might plausibly occur, but only if devices can be designed specifically to exploit this loophole.

As already mentioned, however, the growth of metallic, semiconducting or insulating overlayers on postulated half-metallic substrates (or vice versa) does not necessarily provide grounds for hope for a general 
passivation of the minority-spin surface states. Although they may be altered by contact with the overlayer, it is entirely possible that they will eventually transform into interface states [198-204]. In the case of $\mathrm{NiMnSb}(001)$ discussed above, for example, the deposition of a half-monolayer of $\mathrm{Sb}$ has been shown to severely disrupt the DFT-calculated surface states, but the original eigenfunctions reassert themselves as interface states for coverages of a monolayer and above [199]. Furthermore, the $A_{1}$ symmetry state drops in energy at the zone center, closing the window previously described (see Fig. 9b). Clearly, existing work has barely begun to elucidate the links between surface and interface electronic structure in the halfmetallic materials.

Although the examples discussed above clearly relate to surface phenomena, the principles apply equally well to tunneling across interfaces. In the ideal case, the fully spin-polarized band structure of halfmetallic compounds implies that the electric current should be carried solely by electrons with a certain spin orientation. Half metallic compounds, thus, open tremendous possibilities for device applications such as spin filters and spin injectors [205]. The quality of a prospective spin-valve heterostructure rests upon the nature of any interface states that may exist, and the likelihood of spin-flip scattering through either magnons or open-shell defects. The role of magnons in compromising half-metallicity has been discussed at length elsewhere [167], while in the case of defects the ever-present spectre of interfacial segregation also demands further pause for thought. Neither should one believe that these issues affect only tunnel junctions; even in the Ohmic regime, a high local density of minority-spin interface states still provides ample opportunity for enhanced spin-flip scattering relative to the ideal case.

The most popular choice for electrodes in MTJs has been the perovskite $\mathrm{La}_{0.67} \mathrm{Sr}_{0.33} \mathrm{MnO}$ (LSMO). The initial observation of $82 \% \mathrm{TMR}$ in $\mathrm{LSMO} / \mathrm{SrTiO}_{3} / \mathrm{LSMO}$ MTJs was relatively modest and far from what is expected from the half-metallic nature of LSMO [206]. However, results have been steadily improving. TMR values of more than $400 \%$ at low temperature utilizing $\mathrm{SrTiO}_{3}, \mathrm{PrBaCu}_{2.8} \mathrm{Ga}_{0.2} \mathrm{O}_{7}$, or $\mathrm{CeO}_{2}$ barriers [207,208]. Using Eq. (16), this implies a spin polarization of more than $80 \%$, in agreement with experiments on tunneling to a superconductor [209]. More recently, Bowen et al. [210,211] have observed $1800 \%$ TMR in $\mathrm{LSMO} / \mathrm{SrTiO}_{3} / \mathrm{LSMO}$ junctions, which implies that the spin polarization is $95 \%$ based on Julliere's model. This result was thought to be consistent with the very high values of spin-polarization observed in spin-polarized photoemission [174] and inverse photoemission $[175,212]$ although the latter measurements were taken with limited wave vector sampling and thus not proof that LSMO is half-metallic. Recent analysis of Andreev reflection measurements combined with a re-evaluation of the band structure suggests that LSMO is not half metallic at all $[213,214]$. Improved surface magnetism, as the quality of the interfaces improves, is responsible for this advance as well as the influence of suitable wave vector matching [215]. An extensive review of magnetotransport phenomenon in magnetic oxides can be found in [216].

Other nominally half-metallic compounds, indeed with much higher ground state polarization values, do not show such impressive behavior. For example, $\mathrm{CrO}_{2}$ has been predicted [168] and shown experimentally, using both Andreev reflection [178] and MeserveyTedrow type measurements [179], to be a half metal (although it may not be metallic but rather a semi-metal with high polarization [217]). $\mathrm{CrO}_{2}$ was used as electrodes in $\mathrm{CrO}_{2} / \mathrm{RuO}_{2} / \mathrm{CrO}_{2}$ spin valves, where $\mathrm{RuO}_{2}$ is a metal which has the same rutile structure and very closely matched lattice constant [218]. No appreciable GMR effect was obtained due to a large chemically and magnetically disordered layer at the $\mathrm{CrO}_{2} / \mathrm{RuO}_{2}$ interface. Similarly, TMR of only $14 \%$ was observed in $\mathrm{CrO}_{2} / \mathrm{SnO}_{2} / \mathrm{Co}$ MTJs, where $\mathrm{SnO}_{2}$ is a rutile structure insulator [219]. This result is attributed to structural disorder due to the large lattice mismatch $(\sim 9 \%)$ and $\mathrm{Cr}_{2} \mathrm{O}_{3}$ forming at the interface, but a number of other complications need to be considered as well $[159,185]$.

Another well known material, $\mathrm{Fe}_{3} \mathrm{O}_{4}$, has been predicted to have close to $100 \%$ negative spin polarization $[169,170]$. In practice, the observed TMR values are very modest and even the sign of the spin polarization is not clear. Positive TMR values were reported not only in $\mathrm{Fe}_{3} \mathrm{O}_{4}$ junctions with $\mathrm{AlO}_{\mathrm{x}}$ barriers [220] but also when $\mathrm{SrTiO}_{3}$ was used as a barrier [221]. Recently, small inverse TMR was observed for $\mathrm{Fe}_{3} \mathrm{O}_{4} / \mathrm{SrTiO}_{3} /$ LSMO [222]. Inverse TMR of a few tens of percent was observed in spinel junctions such as $\mathrm{Fe}_{3} \mathrm{O}_{4} / \mathrm{CoCr}_{2} \mathrm{O}_{4} / \mathrm{LSMO}$ [223] and $\mathrm{Fe}_{3} \mathrm{O}_{4} /\left(\mathrm{MgTi}_{2} \mathrm{O}_{4}\right.$ or $\left.\mathrm{FeGa}_{2} \mathrm{O}_{4}\right) / \mathrm{LSMO}$ [224]. Disorder and other phases of $\mathrm{FeO}$, depending on the barrier and the oxidation conditions, at the interface are responsible for this behavior.

Clearly, it does not follow automatically that interfaces involving materials that are nominally halfmetallic in the bulk will have high degree of spin 
polarization. Half metallicity can be suppressed due to reduced coordination of the interface atoms (in addition to the other problems noted above). Formation of different phases at the interface or interface bonding can reduce the spin polarization. In addition, interface states induced by defects and disorder at the interface can further aggravate the problem.

\subsection{Metal-oxide interfaces}

Metal-insulator interfaces play a decisive role in spindependent tunneling in magnetic tunnel junctions where oxides are used as a tunneling barrier [225,118,119]. $A b$-initio calculations of spin-dependent tunneling across $\mathrm{MgO}$ [67] and $\mathrm{ZnSe}$ [226] barriers revealed that the transmission probability becomes very large for certain values of the wave vector $\mathbf{k}_{\|}$parallel to the interface (see Fig. 10). These 'hot spots' were attributed to interface states appearing in the minority $\mathrm{Fe}$ band. The indication of the presence of such resonant states was found in the experiments on epitaxial $\mathrm{Fe} / \mathrm{MgO} / \mathrm{Fe}$ MTJs [227].

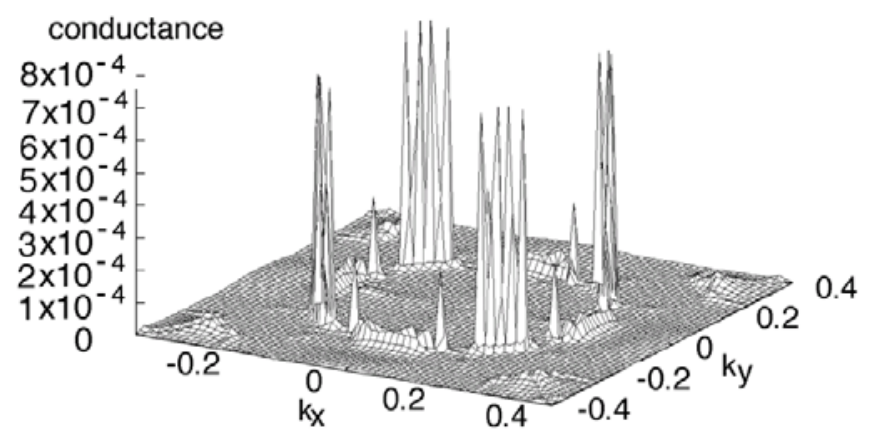

Fig. 10: Minority-spin tunneling transmission through a $\mathrm{ZnSe}$ barrier as a function of $\mathbf{k}_{\|}$in the two-dimensional Brillouin zone for parallel magnetization of Fe electrodes. "Hot spots" are clearly seen. Adopted from [226].

The formation of the 'hot spots' has been explained as follows [139]. When the junction is symmetric the interface states on both sides of the barrier are coupled forming bonding and antibonding states. If the bondingantibonding splitting is larger than the natural resonance width of the interface resonant state, transmission close to unity is observed as the electron goes to the other side of the barrier without tunneling. If the splitting is small, the enhancement in the transmission caused by the interface state may only be due to its larger DOS as compared to propagating states.

For example, the observed reduction of TMR in $\mathrm{Fe} / \mathrm{MgO} / \mathrm{Fe}$ MTJs for small $\mathrm{MgO}$ thickness [66] can be explained by the contribution of interface states to the conductance [228]. With increasing barrier thickness the contribution from interface states may be strongly suppressed due to weak coupling of these states to evanescent states in the barrier. It was also suggested that a layer of $\mathrm{Ag}$ or $\mathrm{Au}$ deposited epitaxially suppresses the transmission through the interface states and significantly improves TMR [229].

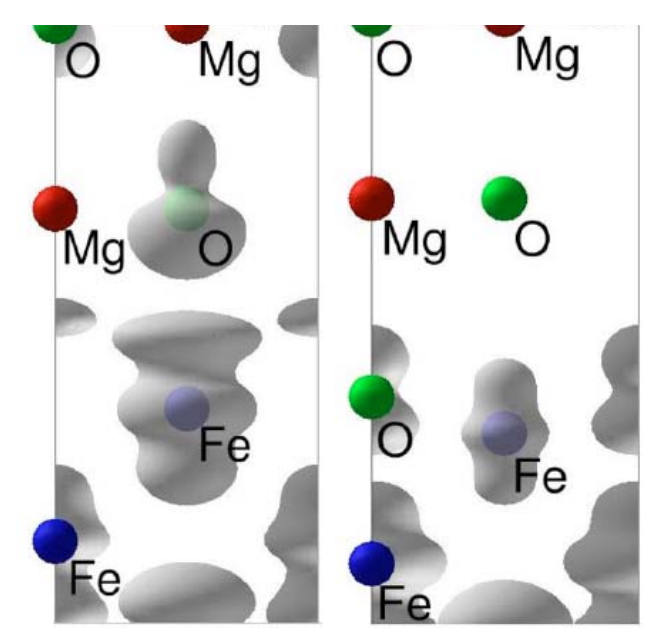

Fig. 11: Partial charge density at the Fermi energy associated with the state of $\Delta_{1}$ symmetry in the majority-spin channel near the interface region. Left panel, without the $\mathrm{FeO}$ layer; right panel, with the $\mathrm{FeO}$ layer. Taken from [230].

The role of oxygen at the interface of $\mathrm{Fe} / \mathrm{MgO} / \mathrm{Fe}$ MTJs [230] was studied in order to explain the discrepancy between the theoretically predicted huge TMR in these systems [67] and the lower TMR observed experimentally. It was found that a monolayer of $\mathrm{FeO}$ at the interface dramatically reduces TMR. Due to the strong bonding between $\mathrm{Fe}$ and $\mathrm{O}$ atoms at the interface the density of states associated with the band of $\Delta_{1}$ symmetry has a tendency to localize within the interface plane reducing the $\Delta_{1}$ character transmitted to $\mathrm{MgO}$ across the interface (see Fig. 11). This spacial localization of states leads to the reduction of TMR as the result of the suppression of the conductance through the $\Delta_{1}$ band controlling the large $\mathrm{TMR}$ in $\mathrm{Fe} / \mathrm{MgO} / \mathrm{Fe}$ junctions. A similar effect was predicted to be produced by boron if it remains at the $\mathrm{FeCo} / \mathrm{MgO}$ interface when the amorphous $\mathrm{FeCoB}$ crystallizes to bcc FeCo upon annealing [231]. Recently, very large TMR was predicted in symmetrically oxidized $\mathrm{Fe} / \mathrm{FeO} / \mathrm{MgO} / \mathrm{FeO} / \mathrm{Fe} \mathrm{MTJ}$ due to interface resonant states [232]. However, perfect matching of the very 
sharp interface resonances is unlikely to happen in practice because the width and position of these states are very sensitive to disorder and/or bias [233].The interface structure was also found to influence strongly the bias dependence of $\mathrm{TMR}$ in $\mathrm{Fe} / \mathrm{MgO} / \mathrm{Fe}$ tunnel junctions [234].

Oxidation of the interfaces was studied also in relation to the problem of the positive spin polarization observed in all ferromagnetic metals in tunneling through $\mathrm{Al}_{2} \mathrm{O}_{3}[69,235]$. Some insights into the mechanisms responsible for the formation of spin polarization can be obtained by considering the electronic structure of ferromagnet/vacuum interfaces. Although the presence of $\mathrm{Al}$ in real $\mathrm{Al}_{2} \mathrm{O}_{3}$-based tunnel junctions will modify the electronic properties of the interface it will not influence dramatically the bonding between the ferromagnet and $\mathrm{O}$, and consequently the mechanism of the inversion of spin polarization. For example, the presence of an $\mathrm{O}$ overlayer on $\mathrm{Fe}$ electrodes was found to invert the spin polarization of the Fe interface [236]. A similar effect was found in the case of a Co surface [113]. In both cases the sign of the spin polarization is reversed from negative for the clean surface to positive for the oxidized surface. Similarly 'adsorption' of $\mathrm{O}$ atoms at the interface between $\mathrm{Co}$ and crystalline $\mathrm{Al}_{2} \mathrm{O}_{3}$ was found to change spin polarization from negative to positive [237]. It was argued that the strong Co-O bonding due to the 'adsorbed' oxygen by the Co electrode is prerequisite for the positive spin polarization observed in the $\mathrm{Al}_{2} \mathrm{O}_{3}$-based tunnel junction experimentally $[69,235]$.

\subsection{Magnetic properties}

The electrostatic potential difference and bonding at the interface cause a flow or sharing of charge through the interface [88] which in turn can change local magnetic properties, in particular the interface magnetic moment and spin polarization.

In general, the magnetic moment of atoms at a surface should increase, due to their reduced coordination number and reduced crystal field splitting making them more similar to stand-alone atoms than atoms in the bulk. However, at the interface of a spinpolarized electrode with a dielectric the magnetic moment of atoms can decrease due to charge transfer and bonding between the magnetic and non-magnetic atoms. Chemisorption of various adatoms or molecules can significantly quench the magnetic moment of atoms in the surface layer, by removal of states from the vicinity of the Fermi level during formation of covalent bonds [238- 242].

For example, the interface Co layer was found to be almost magnetically dead due to oxidation in $\mathrm{Co} / \mathrm{O} /$ vacuum [113] and $\mathrm{Co} / \mathrm{Al}_{2} \mathrm{O}_{3}$ with absorbed $\mathrm{O}$ atoms [237] interfaces. The $\mathrm{Co}-\mathrm{O}$ bond creates bonding and anti-bonding states where the bonding state appears at the Fermi energy and reduces the spin polarization. A similar effect is observed at the $\mathrm{Co} / \mathrm{SrTiO}_{3}$ [123] and $\mathrm{Co} / \mathrm{HfO}_{2}$ [243] interfaces where the magnetic moment on the surface layer is reduced. On the contrary, the magnetic moment of an oxidized Fe electrode increases due to the appearance of an interface state in the minority-spin channel as observed in $\mathrm{Fe} / \mathrm{O} /$ vacuum [236], $\mathrm{Fe} / \mathrm{FeO} / \mathrm{MgO}$ [230], and $\mathrm{Fe} / \mathrm{MgO}$ [228] interfaces. In these cases the magnetization of the interface layer is inverted with respect to the magnetization of the bulk. The magnetically dead layer can invert the transport spin polarization by spin filtering of one of the channels [113]. Similar effects are observed in more complex ferromagnets as well. Charge transfer in $\mathrm{LSMO} / \mathrm{SrTiO}_{3}$ interfaces reduces spin polarization [215]. At $\mathrm{CrO}_{2} / \mathrm{RuO}_{2}$ interfaces, evidence of non-collinear magnetization was found [218]. Such spin polarization reduction diminishes TMR in these systems with postulated half-metallic electrodes from the predicted infinity to much more modest values (Sec. 3.2).

Conversely, bonding at the interface can induce a magnetic moment in the barrier region. Superexchange type interaction between the $\mathrm{Co}$ and $\mathrm{Ti}$ atoms in the $\mathrm{Co} / \mathrm{SrTiO}_{3}$ interface [244] produces a magnetic moment of $0.25 \mu_{B}$ antiparallel to the magnetic moment on the Co. Tunneling of the Co $d$-electrons through the $\mathrm{Ti}$ empty $3 d$ band was found to be the source of negative spin polarization at the $\mathrm{Co} / \mathrm{SrTiO}_{3}$ interface [123]. Similarly, bonding at the $\mathrm{Fe} / \mathrm{BaTiO} 3$ interface can introduce coupling between the ferroelectric and the ferromagnetic ordering parameters i.e. magnetoelectric effect (see Sec. 9).

\section{Interface composition and stability}

In the previous sections, a case for interface criticality was made which focused on the surface and interface electronic structure within the context of model interfaces, most of which are considered abrupt or terminate conveniently. Here, we explore the equilibrium energetics of physical structure to provide a more real picture from which the tunneling spin 
polarization and resultant TMR is perturbed, i.e. the not-so-ideal interface. The section is broken down so as to consider the simple one component electrode/ insulator interfaces as well as the more complex multicomponent alloys which require not only the chemical reactions occurring at the interface with the insulator, but also the bulk versus surface free energy differences (segregation) which can lead to an electrode/insulator interface with multiple stoichiometry regions.

In addition to the phonon and magnon scattering mechanisms described above, scattering by defects may also play a major role in coupling between bulk and surface states. In the absence of crystalline order, the conservation of crystal momentum (as opposed to actual momentum) is no longer a requirement. The effect is familiar from the theory of photoemission, whereby electrons ejected from a well-ordered surface are subject to conservation of crystal momentum in the dimensions parallel to the surface, but not in the dimension of broken symmetry normal to the surface. In the limit of a rough surface or interface, therefore, the picture of well-defined Bloch states provided by band theory becomes invalid, and the wave vector is no longer a good quantum number. When the defect concentration is relatively low, however, one may treat the system as if it were a perfect crystal, including scattering events merely as perturbations in which states of differing crystal momenta may exchange particles. Thus, low-energy defect scattering can, just like phonon scattering, allow electrons and holes to be transferred between surface states at the zone center and bulk states at the zone edge. At the surface, however, the entire boundary may be considered as an extended "defect", so that scattering between surface/interface and bulk states is likely to be rather facile. The consequences for the tunneling current are analogous to the phonon case.

\subsection{Interface composition}

Implicit in the existence of surface states is the free energy created by symmetry breaking at a surface. Indeed, there is a free enthalpy difference between the surface and bulk in both the Heusler alloys [245] and the manganese perovskites [246] and all other metallic systems. This energy difference, plotted for NiMnSb in Fig. 12, is quite significant and leads to surface states, as discussed above, as well as surface reconstructions, changes in chemical reactivity and segregation as indicated in Fig. 13. While these surface and interface effects may share some similarities in origin let us now consider the problems of surface composition and surface segregation.

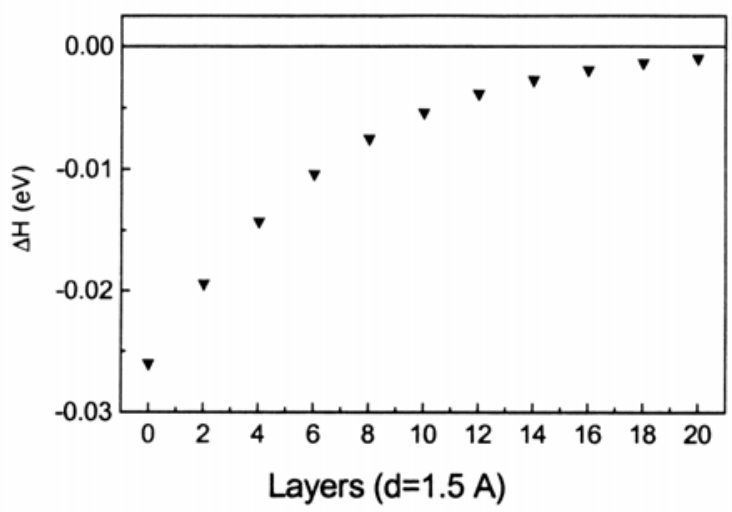

Fig. 12: The free energy difference between the surface and the bulk of stoichiometric NiMnSb, extrapolated from experimental data using simple statistical mechanics models [245].

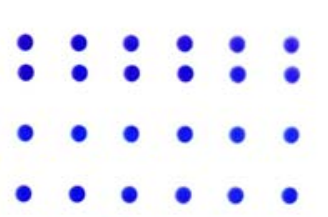

Relaxation

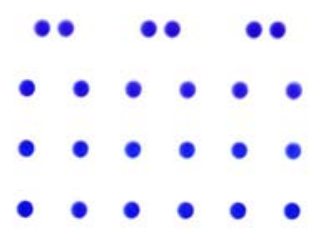

Reconstruction

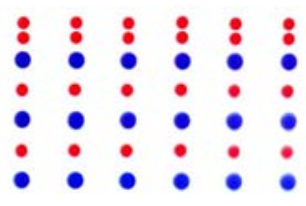

Segregation

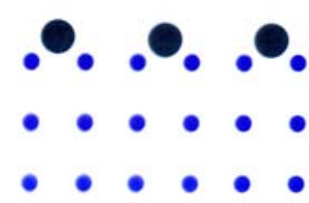

Adsorption
Fig. 13: Schematic representation of reconstructions, segregation and surface reactivity that can occur concomitantly with the presence of electronic surface states.

There is a strong chemical potential for surface segregation in the postulated half-metallic ferromagnetic materials $[245,246]$ that results in equilibrium surfaces at elevated temperatures that are not the same stoichiometry as the bulk. Evidence of the resulting surface segregation has been found for $\mathrm{NiMnSb}(001)$ [245,176,247-249], TiCoSb [248,249] and the manganese perovskites $\mathrm{La}_{1-\mathrm{x}} \mathrm{A}_{\mathrm{x}} \mathrm{MnO}_{3}(\mathrm{~A}=\mathrm{Ca}$, $\mathrm{Sr}, \mathrm{Pb})[246,250$ - 257]. The extent of segregation can be considerable once equilibrium is established and extends from the surface well into the bulk; it need not be localized just to the surface layer. Indeed, in the halfmetallic ferromagnetic systems, at least for those 
systems characterized in detail, surface segregation is rarely restricted to just the surface terminal layers. Variations in composition due to surface segregation can extend often 5 to 10 unit cells away from the surface, as is evident for NiMnSb (detailed in Fig. 14) and the manganese perovskites. It is very clear that for the postulated half-metallic ferromagnets, the stoichiometric surface is rarely stable at finite (elevated) temperatures.

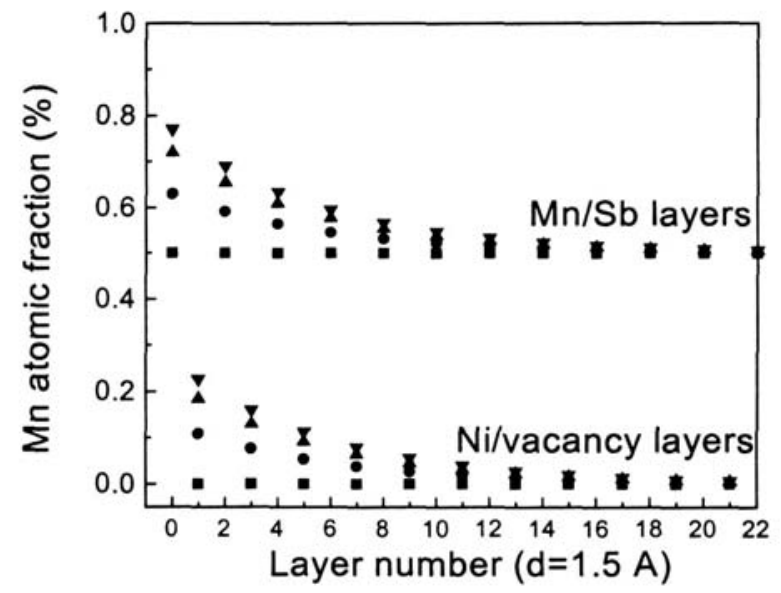

Fig. 14: The concentration of $\mathrm{Mn}$ in the near surface region obtained from fits from the angle resolved X-ray photoemission from [245]. The odd layers are $\mathrm{Mn} / \mathrm{Ni}$. The Mn concentration profiles denoted by the different symbols $(\lambda<\sigma<\tau)$ indicates increasing segregation and filling of vacancies in the near surface region with annealing at $700 \mathrm{~K}$ [245].

The dynamics of surface segregation that include the gradual concentration gradient of one species or another as one approaches the surface and the possible random arrangement of defect sites in the surface layers are far from completely understood. This theoretical problem poses a considerable challenge, but it is clear that as temperature increases, kinetic barriers to segregation are overcome, and are accompanied by a changing free enthalpy of segregation. Thus depolarization due to magnons, phonons, and the nonquasiparticle states that dominates at lower temperatures, is supplanted by even greater depolarization due to compositional changes in the surface region [176,247,253], as seen in Fig. 15, and alluded to in the previous section.

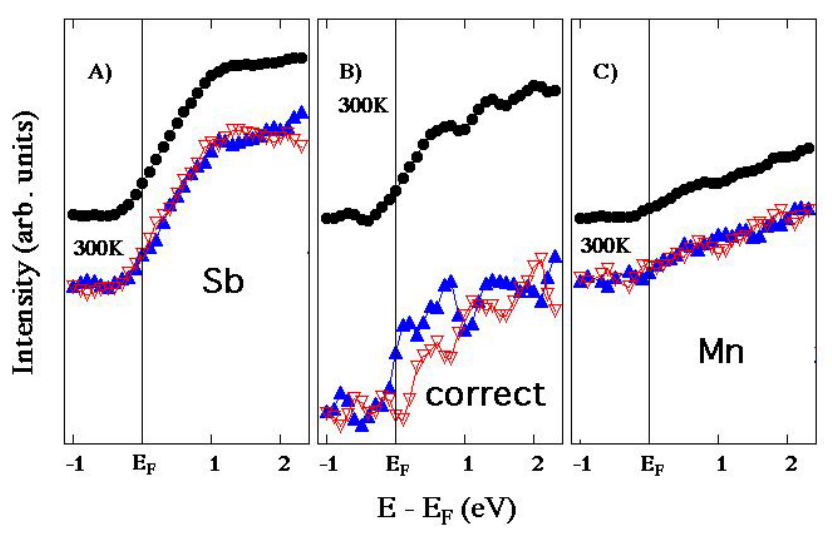

Fig. 15: Spin-polarized inverse photoemission spectra at normal incidence to $\mathrm{NiMnSb}(001)$ with spin-integrated inverse photoemission shown for comparison ( ) probing the unoccupied states above the Fermi energy. (a) For an $\mathrm{NiMnSb}$ surface capped with an Sb overlayer (b) Following the removal of excess $\mathrm{Sb}$ to form the ordered and stoichiometric MnSb terminated (100) surface. (c) Following $\mathrm{Mn}$ segregation to the surface region after (b). $\sigma$-spin up, $\nabla$ spin down are both indicated. Adapted from [176].

Even for postulated half-metallic systems that preserve stoichiometry in the surface region, the composition of the resulting stable surface terminal layer can affect polarization, as noted above. The surface terminal layer can depend not only upon composition of the bulk material, but also on the orientation of the surface layer, even in the absence of segregation. The termination layer of $\mathrm{La}_{0.9} \mathrm{Ca}_{0.1} \mathrm{MnO}_{3}$ is a La-Ca-O layer [246,255] but the termination layer of $\mathrm{La}_{0.65} \mathrm{~A}_{0.35} \mathrm{MnO}_{3}$ has been consistently determined to be an Mn-O layer [251,258-264], except in the case of $\mathrm{La}_{0.65} \mathrm{Sr}_{0.35} \mathrm{MnO}_{3}[250,252]$ where the stable surface region is a different perovskite: a Ruddlesden-Popper phase. There are few experimental studies that compellingly identify the surface composition of manganese perovskite surface terminal layers in the absence of any surface segregation. As a further example, the stable surface of the $\mathrm{CrO}_{2}$ surface is almost always $\mathrm{Cr}_{2} \mathrm{O}_{3}[265,266]$. The complex issue of the stability of compositional stoichiometry cannot be assumed at interfaces, even in absence of segregation. This is particularly true at oxide interfaces.

For complex materials, even the interface termination is an open question. By way of example, let us consider a little more closely the case of NiMnSb, where segregation issues have seriously hampered experimental efforts to produce well-ordered and stoichiometric surfaces. Even concentrating on just the simplest (001) surface facet, we find ourselves faced 
with non-trivial questions of surface composition. The surface could conceivably possess two differing surface terminations, ending on either a $\mathrm{Ni}$ layer or on a $\mathrm{MnSb}$ layer. From an experimental perspective the preparation of any well-ordered surface of this type is fraught with difficulty, although characterization of the surface indicates a stoichiometric MnSb-termination is favored $[176,245]$.

\subsection{Interfaces with oxides: oxidation/reduction reactivity}

Assume the canonical electrode/insulator combination $\mathrm{Co} / \mathrm{Al}_{2} \mathrm{O}_{3}$, where the cobalt is grown to provide an atomically abrupt $h c p$ structure with amorphous, but stoichiometrically consistent, alumina deposited on top. Further, assume that the method, through which the alumina is formed, occurs at $10^{-12}$ Torr and does not cause the ejection of cobalt, growth of imperfections or component mixing, such that an ideal interface initially exists. From the instant such an interface is formed, oxygen begins to displace and chemically reduce the aluminum so as to form $\mathrm{CoO}$ at the interfacial layer [267-269], which in turn causes a stoichiometric deficiency in the $\mathrm{Al}_{2} \mathrm{O}_{3}$ matrix. The end result is a picture of the interface best described as $\mathrm{Co} / \mathrm{CoO} / \mathrm{AlO}_{\mathrm{x}} / \mathrm{Al}_{2} \mathrm{O}_{3}$. The same is true of the $\mathrm{Fe} / \mathrm{MgO}$ interface [270] and for CoFe/oxide interface [271]. The implications of such a simple interfacial displacement are deleterious to not only the spin polarization but also the tunneling current and hence the overall TMR. The spin polarization is affected because $\mathrm{CoO}$ is an antiferromagnetic insulator which depolarizes the bulk Co minority dominated polarization [272]; an analogous structure is the $\mathrm{Co}$ interface with $\mathrm{NiO}$ $[272,273]$. Also, the tunneling current magnitude is reduced as well because the real space distance which makes up the barrier is now increased to include the insulating $\mathrm{CoO}$ layers, which extend at least $10 \AA$ [274276], not counting both interfaces; the barrier height has also been shown to increase [268]. Other examples of barrier width increase from $\mathrm{Co}_{2} \mathrm{MnSi}$ exist as well [277]. Further, if interfacial mixing occurs, symmetry breaking will lead to spin reorientations [273] that may further reduce the tunneling spin polarization.

The predicate of barrier reduction and electrode oxidation is the difference in free energy, wherein the larger free energy metal oxide will dominate or acquire the oxygen. Table 1 lists the free energy values of various metal oxides of application interest. From this table it is immediately obvious that the oxide insulators which we generally regard as refractory, will indeed form interfacial oxides with common and alloy transition metal injectors.

Not only does oxide formation with transition metals cause antiferromagnetic ordering at the interface that can reduce spin polarization, but the oxide can also initiate an exchange anisotropy [278-280,276] as a result of combining an ferromagnet with an antiferromagnet (which occurs at the new interface with unoxidized electrode). This anisotropy can result in pinning, at an interface that should otherwise be rotatable, i.e. $\mathrm{Al}_{2} \mathrm{O}_{3} / \mathrm{AlO}_{\mathrm{x}} / \mathrm{NiO} / \mathrm{NiFe}$.

These complications due to interface chemistry are not all negative: there are recent results for $\mathrm{Fe}$ on $\mathrm{NiO}$ [281] which find that interfacial buckling $(0.3 \AA)$ and expansion $(7 \%)$ due to $\mathrm{Fe}-\mathrm{O}$ formation, can cause an increase in moment $\left(0.6 \mu_{\mathrm{B}}\right)$, begging the question as to whether there is a commensurate increase in polarization. With regard to increased moment due to interfacial oxidation, there are some [282] who argue that such induced moment is absent based on empirical data. Recently, experimental [267,283,284] and theoretical work $[237,285,286]$ has shown that there is an optimum level of interfacial oxidation where TMR reaches a maximum. Such work has been completed for $\mathrm{Co} / \mathrm{Al}_{2} \mathrm{O}_{3}$ and $\mathrm{Fe} / \mathrm{MgO}$ interfaces, where each system provides similar results, despite rather disparate physical ordering, i.e. amorphous $\mathrm{Al}_{2} \mathrm{O}_{3}$ versus the more crystalline $\mathrm{MgO}$. Such optimal interfacial oxidation conditions, however, do not surpass the TMR that can be obtained with an ideal non-oxide based interface [287]. Section 8 will discuss the possibility for novel insulators that may form such abrupt and symmetry conserving interfaces. One should also question whether such an optimal interfacial oxide is stable; that is, whether the $\mathrm{Co} / \mathrm{CoO} / \mathrm{AlO}_{\mathrm{x}} / \mathrm{Al}_{2} \mathrm{O}_{3}$ combination is at its lowest free energy state. While the mechanism responsible for increased TMR in optimally oxidized interfaces is not yet known, it is known that spin-flip scattering due to a non-optimal oxide causes deleterious TMR [288]. The free energy/enthalpy argument can be somewhat flawed however, as evidenced by the oxidation of $\mathrm{Fe}$ at a $\mathrm{SiO}_{2}$ interface [289], wherein the $\mathrm{Si}$ is thermodynamically favored.

Table 1: Gibbs free energy of formation for selected compounds. All energies are given at $298 \mathrm{~K}$. Adopted from Ref. [290].

\begin{tabular}{|c|c|c|c|}
\hline Oxide & $\Delta \mathbf{G}$ & Oxide & $\Delta \mathbf{G}$ \\
\hline
\end{tabular}




\begin{tabular}{|c|c|c|c|}
\hline $\mathrm{Al}_{2} \mathrm{O}_{3}$ & -1582.3 & $\mathrm{MnO}$ & -362.9 \\
\hline $\mathrm{As}_{2} \mathrm{O}_{5}$ & -782.3 & $\mathrm{MoO}_{2}$ & -533.0 \\
\hline $\mathrm{B}_{2} \mathrm{O}_{3}$ & -1194.3 & $\mathrm{MoO}_{3}$ & -668.0 \\
\hline $\mathrm{BaO}$ & -520.3 & $\mathrm{NiO}$ & -211.7 \\
\hline $\mathrm{CaO}$ & -603.3 & $\mathrm{P}_{4} \mathrm{O}_{10}$ & -2723.3 \\
\hline $\mathrm{Co}_{3} \mathrm{O}_{4}$ & -744.0 & $\mathrm{~Pb}_{3} \mathrm{O}_{4}$ & -601.2 \\
\hline $\mathrm{CoO}$ & -241.2 & $\mathrm{PbO}$ & -188.5 \\
\hline $\mathrm{Cr}_{2} \mathrm{O}_{3}$ & -1058.1 & $\mathrm{PbO}_{2}$ & -217.3 \\
\hline $\mathrm{CrO}_{2}$ & -598.0 & $\mathrm{Sb}_{2} \mathrm{O}_{5}$ & -829.2 \\
\hline $\mathrm{Fe}_{2} \mathrm{O}_{3}$ & -742.2 & $\mathrm{SiO} 2$ & -856.3 \\
\hline $\mathrm{Fe}_{3} \mathrm{O}_{4}$ & -1015.4 & $\mathrm{SiO}_{2}-$ & -801.2 \\
\hline $\mathrm{FeO}$ & -251.4 & $\mathrm{SiO}_{2}-$ & -783.2 \\
\hline $\mathrm{FeS}_{2}$ & -166.9 & $\mathrm{SrO}$ & -561.9 \\
\hline $\mathrm{La}_{2} \mathrm{O}_{3}$ & -1705.8 & $\mathrm{Ti}_{3} \mathrm{O}_{5}$ & -2317.4 \\
\hline $\mathrm{MgO}$ & -569.3 & $\mathrm{TiO}$ & -495.0 \\
\hline $\mathrm{Mn}_{2} \mathrm{O}_{3}$ & -881.1 & $\begin{array}{l}\mathrm{TiO}_{2} \\
\text { (anatase) }\end{array}$ & -884.0 \\
\hline $\mathrm{Mn}_{3} \mathrm{O}_{4}$ & -1283.2 & $\begin{array}{l}\mathrm{TiO}_{2} \\
\text { (rutile) }\end{array}$ & -890.0 \\
\hline $\mathrm{MnO}_{2}$ & -465.1 & $\mathrm{Ti}_{2} \mathrm{O}_{3}$ & -1434.0 \\
\hline
\end{tabular}

Beyond the single component electrodes are the oxide electrodes, such as $\mathrm{CrO}_{2}$ and $\mathrm{Fe}_{3} \mathrm{O}_{4}$, that are halfmetals from ground state calculation $[168,169]$, but far from $100 \%$ spin-polarization by experiment near room temperature. The relevance here is not to argue for or against half-metallicity, but exploit the interfacial stoichiometry of $\mathrm{Fe}$ and $\mathrm{Cr}$, when in an oxygen rich or depleted environment. For $\mathrm{CrO}_{2}$, the native $\mathrm{Cr}_{2} \mathrm{O}_{3}$ readily forms as the thermodynamic sink for $\mathrm{CrO}_{2}$ in the presence of excess oxygen as easily supplied by an oxide insulator with $\mathrm{Cr}$ providing the larger affinity for oxygen compared to $\mathrm{Al}$ or $\mathrm{Mg}$. From a tunneling view, $\mathrm{Cr}_{2} \mathrm{O}_{3}$ is an antiferromagnetic insulator, which again destroys polarization [359]. For $\mathrm{Fe}_{3} \mathrm{O}_{4}$ a more complicated thermodynamic sink exists, wherein $\mathrm{FeO}$, $\alpha-\mathrm{Fe}_{2} \mathrm{O}_{3}$ and $\beta-\mathrm{Fe}_{2} \mathrm{O}_{3}$ may co-exist despite the free energy differences [291,292], wherein anisotropy arguments must be considered.

\subsection{Metal alloy segregation}

Segregation, or the enrichment of a multicomponent solid at a grain boundary, interface or surface due to free energy differences [293] is, like oxidation, critical to understanding TMR, as once again, the spin polarized electron must cross this otherwise non-ideal interface. We review here two popular electrode systems, that both have promised to revolutionize magnetic tunnel junctions through their predicted halfmetallicity. These systems are the manganese perovskites and the full and half-Heusler alloys.

\subsubsection{Perovskites}

Many reviews [249,294,295] and papers [176,245-248, 250-254,258,266,296-297] now exist which detail the implications of segregation on spin polarization at the surface of two to four component alloys. Temperature in this context may be used as an initiator of segregation or an increase in phonon-electron-magnon coupling [76], which in either case causes TMR reduction, but is subtly and importantly different. At the lower temperatures for ideal crystals and interfaces, the proposed half-metallic compounds win out, providing polarizations on the order of $98 \%$, while at higher temperatures, the difference in DOS at $\mathrm{E}_{\mathrm{F}}$ quickly fade. However, at higher temperatures, sometimes hit by industrial operation $\left(70^{\circ} \mathrm{C}\right)$ and fabrication anneals $(230$ ${ }^{\circ} \mathrm{C}$ ), the rate of segregation is enhanced. Such temperature induced implications for polarization beg the question as to whether the simple single component transition metals actually hold the largest polarization value at room and operational temperature, thereby casting doubt on the need for investigations of complex compound materials. One caveat that ought to be recalled from the previous section, however, is the degraded interfacial polarization for single-component electrodes matched up with oxide interfaces. Here, the single component material is susceptible to interfacial depolarization and increasing the tunnel barrier width, whereas with some of the multi-component electrodes a more stable interface may exist due to the equilibrium oxygen content already present at a segregated surface or interface.

\subsubsection{Heusler alloys}

Both the full and half-Heusler compounds have been predicted as half-metallic. Recent experimental work has demonstrated that interfacial admixtures of the Heusler alloys with various insulators indeed occur and again reduce spin polarization across such interfaces. One blatant example is that of the $\mathrm{Co}_{2} \mathrm{MnSi} / \mathrm{Al}_{2} \mathrm{O}_{3}$ interface in which $\mathrm{Mn}_{2} \mathrm{SiO}_{4}$ is shown as having a more favorable enthalpy of formation, and indeed this compounds does in fact occur during plasma oxidation to a thickness of $6 \AA[298,299]$. The implication of this interfacial $\mathrm{Mn}_{2} \mathrm{SiO}_{4}$ is not only an increased barrier 
width, but also various barrier heights along the width due to asymmetry and a dramatically reduced moment as proven by XMCD results. Also, for $\mathrm{Co}_{2} \mathrm{MnSi}$ as well as for NiMnSb, segregation by means of atomic displacement (e.g. Co swapped for $\mathrm{Mn}$ ) has been found as a means of reducing the surface moment and polarization [300-302]. Beyond segregation or oxidation, the filling of antisites in $\mathrm{Co}_{2} \mathrm{MnSi}$, especially in the case of Co antisites, was found [302,303] to not only be likely, from enthalpy considerations, but also was calculated to destroy any half-metallicity.

Studies on $\mathrm{Co}_{2} \mathrm{Cr}_{0.6} \mathrm{Fe}_{0.4} \mathrm{Al}$ reveal greatly reduced moments for $\mathrm{Cr}$ and $\mathrm{Fe}$ and resultant loss of halfmetallic character upon annealing, attributed to increased atomic disorder [304] and possible swapping of $\mathrm{Al}$ with $\mathrm{Co}$ [305].

For $\mathrm{NiMnSb}(100)$, the surface concentration profiles were easily constructed from angle resolved core level photoemission data [245]. Fig. 14 presents the $\mathrm{Mn}$ concentration dependence upon layer number for the three heavily annealed surfaces. Note that the same figure also contains the constant $\mathrm{Mn}$ atomic fraction of 0.5 for the $\mathrm{Mn} / \mathrm{Sb}$ layers and 0 for the $\mathrm{Ni}$ /vacancy layers, characteristic of a stoichiometric structure for the freshly prepared surface. This surface termination of $\mathrm{MnSb}$, for the stoichiometric surface is entirely consistent with the predictions of Jenkins and co-workers [159,199,306,307].

When equilibrium is established between the surface and the bulk, the surface enthalpy is quite different from the bulk $[163,267]$. Surface segregation is a strong indication that the surface enthalpy differs significantly from the bulk, in the context of standard statistical models. We can calculate the dependence of enthalpy as a function of layer number, as demonstrated in Fig. 12, for the most extensively Mn segregated surface $[163,267]$. This difference in enthalpy can be related to the creation of a surface electronic structure very different from the bulk (i.e. surface states for the stoichiometric surface), can result in a new surface lattice structure distinct from the bulk and, of course, can become the driving force for segregation, as indicated schematically in Fig. 13. This energy difference, between the surface and the bulk, appears to be more than sufficient to overcome the energy barriers to defect formation, that have been thought to hinder defect mediated reduction in polarization $[308,309]$.
Most theoretical work on tunneling is devoted to epitaxial systems with ideal interfaces. At the same time the majority of tunneling experiments are performed with amorphous barriers, such as $\mathrm{Al}_{2} \mathrm{O}_{3}$, where disorder plays an important role. Only recently, experimentalists have managed to produce very high quality epitaxial MTJs, such as $\mathrm{Fe} / \mathrm{MgO} / \mathrm{Fe}$ [66]. However, even in these systems there is evidence for a substantial amount of defects, the most common of thich are $\mathrm{O}$ vacancies [310,311]. Fig. 16 demonstrates results of scanning tunneling spectroscopy measurements of the differential conductance of a textured $\mathrm{MgO}$ film grown on $\mathrm{Fe}(001)$. Peaks within the band gap of $\mathrm{MgO}$ (indicated by arrows in Fig. 16) are clearly seen, associated with defect states in $\mathrm{MgO}$. According to the analysis performed in Ref. 312 , the peaks indicated by the solid lines are due to the $\mathrm{O}$ vacancy ground s-state and the peaks indicated by the dashed lines are due to the $\mathrm{O}$ vacancy excited $p$-state. The charge densities corresponding to these states are shown in Fig. 17a,b.

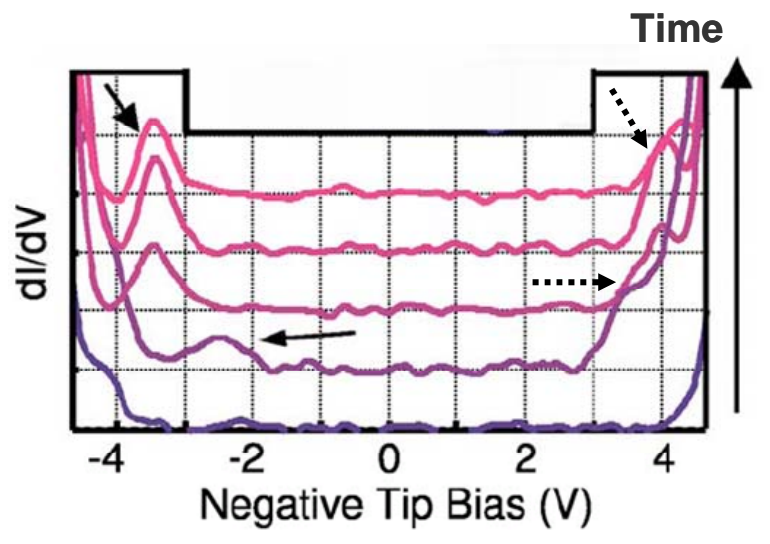

Fig. 16: Scanning tunneling spectroscopy measurements of the differential conductance as a function of bias voltage for $30 \AA$ (001) textured $\mathrm{MgO}$ film grown on (001) Fe. The initial curve exhibits very close to the full $7.8 \mathrm{eV} \mathrm{MgO}$ bulk band gap, while defect states (see arrows) appears in subsequent measurements as the STM tip drifts. Adapted from Ref. 311. According to the analysis performed in Ref. 312 the peaks indicated by the solid and dashed lines can be attributed to $\mathrm{O}$ vacancy $s$ - and $p$ - states respectively.

\subsection{Defects in the barrier}



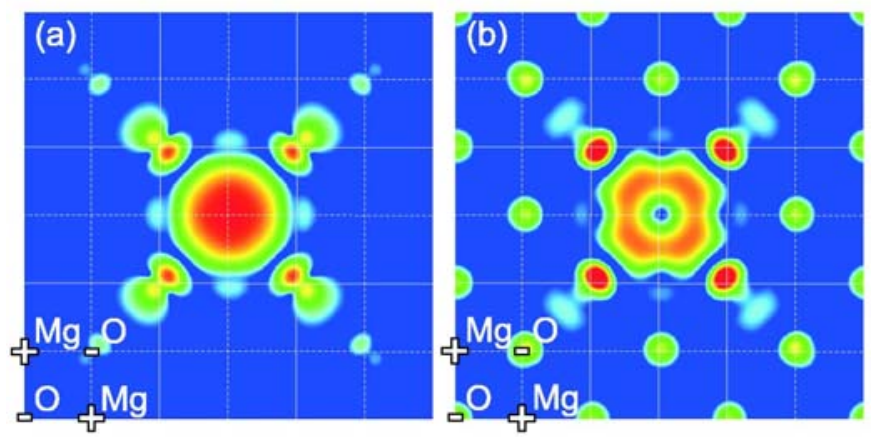

Fig. 17: Calculated charge density corresponding to the $s$ state (a) and $p$-state (b) of the $\mathrm{O}$ vacancy in bulk $\mathrm{MgO}$. Vacancy is located in the middle of the unit cell, the positions of the $\mathrm{Mg}$ and $\mathrm{O}$ ions are indicated. Exponential scale is used with red indicating high and blue low density. Note that due to the location of the $p$-state within the conduction band of $\mathrm{MgO}$ the $p$-state charge density is distributed over the whole unit cell. Taken from Ref. 312 .

Resonant tunneling was proposed to play an important role in disordered MTJs [140, 313, 314]. Also resonant tunneling was predicted to cause a reversal of TMR [315]. Such a reversal was found in $\mathrm{Ni} / \mathrm{NiO} / \mathrm{Co}$ nanowire MTJs [316] where the small cross-section of these junctions allowed for tunneling through a single impurity state. In an ensemble of samples prepared under identical conditions, some of the sampled showed normal and some inverse TMR, which could only be explained by random defects and not by the structure of the interface. Recently a variation of TMR produced by resonant tunneling was observed in junctions based on carbon nanotubes [26]. Defect mediated tunneling was argued to play a decisive role in $\mathrm{CrO}_{2} / \mathrm{Cr}_{2} \mathrm{O}_{3} / \mathrm{CrO}_{2}$ junctions [317,318].

If the defect level does not match the chemical potential in the electrodes it can still affect the tunneling. The presence of defects reduces the mobility and the hopping (one needs next nearest neighbor hopping to go around). Therefore, defects effectively increase the effective mass and decay rate. For example, it was found that $\mathrm{O}$ vacancies in the bulk of $\mathrm{MgO}$ have a profoundly negative effect on TMR in $\mathrm{Fe} / \mathrm{MgO} / \mathrm{Fe}(001) \mathrm{MTJs}$ [312].

Resonant tunneling through $\mathrm{O}$ vacancies may be responsible for the reduction of TMR [312,319]. Giant TMR values of more than $1000 \%$ were predicted theoretically for ideal $\mathrm{Fe} / \mathrm{MgO} / \mathrm{Fe}(001)$ MTJs $[67,68]$. These values differ significantly from more modest TMR values of up to $300 \%$ obtained experimentally in MgO-based MTJs utilizing both epitaxial and textured $\mathrm{Fe}$ and $\mathrm{CoFe}$ electrodes $[65,66]$. First-principles calculations show that $\mathrm{O}$ vacancies can substantially reduce TMR [312,319]. For ideal junctions, TMR is largely controlled by the enhanced transmission of the majority-spin band. The conductance through a defective $\mathrm{MgO}$ barrier displays two distinct transport regimes - resonant tunneling when the electron energy is close to the position of the vacancy levels and nonresonant tunneling when the electron energy is far from the vacancy levels. At resonance, the conductance asymmetry arises only from the difference in the majority and minority DOS in the electrodes, which is fairly small. Non-resonance scattering from vacancies diminishes the spin asymmetry by effectively increasing the decay rate of the majority-spin channel. Overall, $\mathrm{O}$ vacancies cause a substantial reduction of TMR compared to the ideal case. Recently, the inversion of TMR and the interface spin polarization in $\mathrm{La}_{2 / 3} \mathrm{Sr}_{1 / 3} \mathrm{MnO}_{3} / \mathrm{SrTiO}_{3} / \mathrm{Co}$ MTJs was also attributed to resonant tunneling through $\mathrm{O}$ vacancies in the barrier [320].

Resonant tunneling through defect states can be used to explain abnormal behavior on the interlayer exchange coupling across a tunnel barrier. Results obtained in experiments on MTJs [43,44] significantly differ from the theoretical predictions $[46,49]$ both in the magnitude and the sign of the interlayer exchange coupling. Changes in the DOS within the spacer layer have crucial importance for the strength of the interlayer coupling [49]. Therefore, the presence of impurities or defects in the barrier may significantly influence the coupling. In the framework of a free electron model, the interlayer exchange coupling was calculated for a rectangular barrier using the spin torque approach [48]. A defect level was approximated by a $\delta$ function inside the barrier. It was shown that the resonant origin of the impurity-assisted coupling can make the interlayer exchange coupling antiferromagnetic in agreement with the experimental observations $[43,44]$. Recently, the interlayer exchange coupling was calculated using ab-initio calculations of the ground state energy of the MTJ for parallel and antiparallel configuration of the magnetization in $\mathrm{Fe} / \mathrm{MgO} / \mathrm{Fe}(001) \mathrm{MTJs}$ [45]. The coupling was found to be ferromagnetic for ideal $\mathrm{MgO}$ and antiferromagnetic in the presence of oxygen vacancies for small barrier thicknesses. The change in sign of the coupling is produced by spin-dependent broadening of the vacancy level due to interaction with the electrodes. An effect of defects in the barrier on interlayer exchange coupling was also found in $\mathrm{Co} / \mathrm{Cr}_{2} \mathrm{O}_{3} / \mathrm{CrO}_{2}$ [321] and in $\mathrm{Fe}_{2} \mathrm{O}_{3} / \mathrm{MgO} / \mathrm{Fe}$ junctions [322]. 


\section{Ferroelectric/ferromagnetic interfaces}

Reducing the thickness of a ferroelectric film can cause the polarization to disappear due to depolarizing fields produced by polarization charges accumulated at the surfaces of the film. The critical thickness for ferroelectricity in thin films was thought to be much larger than the thickness necessary for tunneling to take place. However, when the ferroelectric film is placed between two metal electrodes, the polarization charges are screened by carriers from the metal. Recent experimental [323-325] and theoretical [326-328] studies of perovskite ferroelectric oxides demonstrate that ferroelectricity persists down to a nanometer scale. For a recent comprehensive review of the physics of thin-film ferroelectrics see Ref. 329.

\subsection{Ferroelectric tunnel junctions}

The existence of ferroelectricity in nm-thick films makes it possible to use them as barriers in ferroelectric tunnel junctions (FTJ) [78]. As was discussed in Sec. 2.4, FTJs may exhibit a giant electroresistance (GER) effect $[79,80,340]$ associated with the polarization switching of the ferroelectric barrier layer. While the electronic origin of the effect is not unquestionably established, hysteretic behavior of the resistance in electric field has been observed in $\mathrm{Pt} / \mathrm{SrRuO}_{3} / \mathrm{Pb}\left(\mathrm{Zr}_{0.52} \mathrm{Ti}_{0.48}\right) \mathrm{O}_{3} / \mathrm{Pt}$ junctions [81].

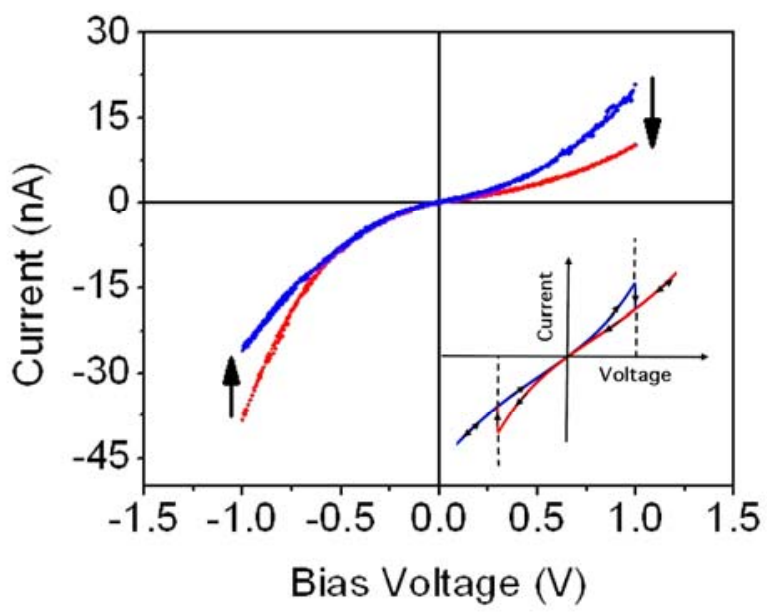

Fig. 18: Reproducible current differences in the $I-V$ curves are seen at small bias in changing the applied voltage from $15 \mathrm{~V}$ to $+15 \mathrm{~V}$ (blue) and $+15 \mathrm{~V}$ to $-15 \mathrm{~V}$ (red) in heterojunctions formed from $4 \mathrm{~nm}$ of CuPc deposited on 3 $\mathrm{nm}$ of $\mathrm{P}(\mathrm{VDF}-\mathrm{TrFE})$. A schematic of the expectation $[80,330]$ for a ferroelectric tunnel junction barrier are indicated in the inset as adapted from [330].
Similar observation was made in semiconducting copper phthalocyanine $(\mathrm{CuPc})$ and organic ferroelectric material polyvinylidene fluoride with trifluoroethylene (or P(VDF-TrFE)) in the electric field controlled $p-n$ diode formed by combining these two molecular thin films together [331]. Biasing the diode by $\pm 15 \mathrm{~V}$, yields hysteresis behavior in the current versus voltage traces at low bias, as seen in the insert to Fig. 18. Application of bias voltages up to $\pm 15 \mathrm{~V}$ is more than sufficient to polarize the ferroelectric $\mathrm{P}(\mathrm{VDF}-\mathrm{TrFE})$ [332], and/or flip the dipole direction of P(VDF-TrFE) (or both $\mathrm{P}(\mathrm{VDF}-\mathrm{TrFE})$ and copper phthalocyanine) thin films of this thickness, so this is not entirely unexpected. Unfortunately, the dipole interaction could affect the diode properties in several ways, which are difficult to distinguish from these measurements. The local electric field due to the P(VDF-TrFE) could also align the copper phthalocyanine layer dipoles and either change the molecular orientation(s) or change the molecular dipole alignment(s) at the interface thus leading to a decrease or increase in the barriers to current [331]. Changes in the barrier height could produce dramatic changes in the tunneling current [78,340], and must be considered likely as both organic films are quite thin (to exclude final state effects and charging). Alternatively, changes in dipole orientation or dipole alignment could change the molecular orbital alignment of the copper phthalocyanine molecular layer relative to $\mathrm{P}(\mathrm{VDF}-$ TrFE), resulting in an effective change in the 'bandoffsets' and the diode characteristics, and cannot be excluded. Still, this does show the possible promise of a ferroelectric spacer layer, even though the structure was in no way magnetic [331].

Expectations on what should be the behavior of a ferroelectric tunnel junction barrier, based on the Brickman model [333], have been much discussed and these expectations are borne out in the P(VDF-TrFE) $\mathrm{CuPc}$ heterojunction device, as indicated in Fig. 18. Other ferroelectric tunnel junction devices, using inorganic ferroelectric barrier layers, also show hysteresis but the hysteresis for the P(VDF-TrFE) $\mathrm{CuPc}$ heterojunction device is reversed from some [81,330,334,335], but not all [336], inorganic ferroelectric barrier layers reported elsewhere. The hysteretic current-voltage (I-V) characteristics may differ from the P(VDF-TrFE) - CuPc heterojunction device shown here because of space-charge-limitedcurrent conduction with the inorganic ferroelectric barrier layers [334].

There are several effects which complicate the physics of FTJs as compared to MTJs. First, 
ferroelectricity is strongly affected by bonding at the interface. In displacement ferroelectrics, such as perovskite oxides, the electric dipole is produced by shifting of atoms within the unit cell from their high symmetry positions. The presence of interfaces imposes restrictions on the atomic displacement since the atoms at the boundary of the ferroelectric are pinned to the electrodes. Thus, ferroelectric polarization in thin films is a superposition of a soft mode and interface polarization dipoles. This effect was demonstrated in $\mathrm{KNbO}_{3}$ ferroelectric films placed between $\mathrm{SrRuO}_{3}$ or $\mathrm{Pt}$ electrodes [337], as well as $\mathrm{BaTiO}_{3}$ with $\mathrm{Fe}$ [338] and Pt [340], and $\mathrm{SrRuO}_{3}[328,339]$ electrodes.

Second, incomplete screening of the polarization charges at the interface of FTJs create finite size charge depletion regions at the interfaces as shown in Fig. 19. In FTJs with different electrodes the potential profile is asymmetric with respect to the polarization orientation in the ferroelectric layer producing the GER effect [79]. Evidence of the GER effect induced by interface bonding was demonstrated in first-principles transport calculations of $\mathrm{Pt} / \mathrm{BaTiO}_{3} / \mathrm{Pt}$ FTJs showing that the interface transmission function of the $\mathrm{Pt} / \mathrm{BaTiO}_{3}$ interface can differ by a factor of three depending on the polarization direction [340].

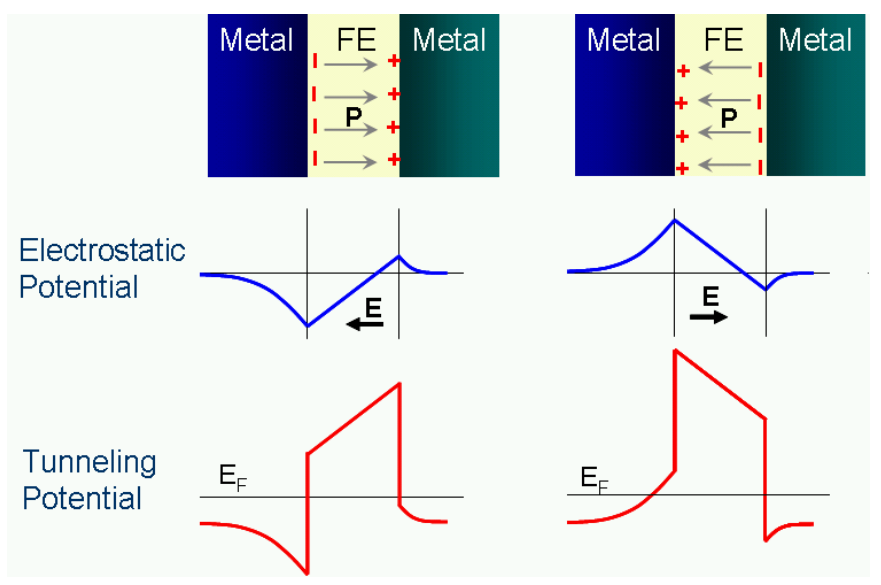

Fig. 19: Ferroelectric tunnel junction in which two metal electrodes are separated by a ferroelectric barrier. Polarization charges for two opposite polarization orientations (top panels), shapes of the electrostatic potential profile associated with depolarizing field (middle panels), and tunneling potential profiles seen by transport electrons (bottom panels).

Last, ferroelectricity is intimately related to lattice distortions. Therefore, most ferroelectrics are piezoelectric as well and applied bias will cause distortion of the barrier layer. Tetragonal expansion/contraction along the axis of the junction will change the barrier thickness and the atomic displacement at the interfaces which will influence the decay rate in the barrier and the interface transmission functions respectively (Eq. 13). At the same time this will be accompanied by contraction/expansion in the plane of the interface which would apply strain on the electrodes possibly influencing their magnetic moment and anisotropy.

\subsection{Multiferroic tunnel junctions}

Recently, a MFTJ based on $\mathrm{BiMnO}_{3}$, which is a bulk multiferroic material, was shown to have four resistance states [82]. An alternative to single phase multiferroics is to combine simple ferroic materials in a layered heterostructure. First principles calculations have been performed on $\mathrm{Fe} / \mathrm{BaTiO}_{3} / \mathrm{Fe}$ MFTJs showing a large change of the interface magnetic moment $\left(\Delta M_{\mathrm{Ti}}=0.25 \mu_{\mathrm{B}}\right)$ with switching the polarization direction i.e. magnetoelectric effect [341]. This is due to change in the $\mathrm{Fe}-\mathrm{Ti}$ bond polarization at the interface driven by ferroelectric displacements. First principles transport calculation of $\mathrm{Fe} / \mathrm{BaTiO}_{3} / \mathrm{Fe}$ MFTJs [341] show that ferroelectric displacements affect differently the interface transmission of the magnitude for parallel and antiparallel orientation of the magnetization of the electrodes. In a junction with asymmetric interfaces this could lead to electric control of the magnetoresistance in MFTJs.

The application of multifunctional materials is not limited to the tunnel junction setup. Control over the spin polarization of the current was proposed by means of ferroelectric films on top of ferroelectric electrodes. The spin polarization of the current was demonstrated to switch between two different states by switching the electric polarization in the ferroelectric [342]. First principles transport calculations of $\mathrm{Fe} / \mathrm{BaTiO}_{3} / \mathrm{Fe}$ MFTJs show that ferroelectric displacements affect differently the interface transmission of the magnitude for majority- and minority-spin channels [341]. Recent experimental studies found reversible changes in magnetic properties of an $\mathrm{Fe}$ thin film placed in proximity of a $\mathrm{BaTiO}_{3}$ single crystal [343]. Large magnetization changes emerged in response to ferroelectric switching and structural transitions of $\mathrm{BaTiO}_{3}$ controlled by applied electric fields and temperature. Interface strain was identified as the primary mechanism responsible for the effect. These 
results indicate the possibility of electrical control of the spin polarization.

Conversely, one can consider a thin magnetic film on top of a ferroelectric substrate. It is predicted that magnetic anisotropy of a thin magnetic film may be tailored by electric means through proximity to a ferroelectric material. Using a $\mathrm{BaTiO}_{3} / \mathrm{Fe}$ bilayer as a representative model and performing first-principles calculations some of the present authors have demonstrated that a reversal of the electric polarization of $\mathrm{BaTiO}_{3}$ produces a sizeable change in magnetic anisotropy energy of $\mathrm{Fe}$ films [344]. Tailoring the magnetic anisotropy of a magnetic film by an adjacent ferroelectric material may yield entirely new device concepts, such as electric-field assisted magnetic data storage.

\subsection{Other applications}

In many applications the interface between a ferromagnet and an antiferromagnet is used to 'pin' the magnetic moment of the ferromagnet. This phenomenon, known as exchange bias, is caused by exchange coupling at the interface between the two materials. Recently, it was shown that interfaces of ferromagnets to multiferroic materials, such as $\mathrm{BiFeO}_{3}$, which is antiferromagnetic and ferroelectric, can cause an electric control of the exchange bias [345]. Magnetoelectric switching of the exchange bias has been demonstrated in $\mathrm{Cr}_{2} \mathrm{O}_{3}(111) /(\mathrm{Co} / \mathrm{Pt})_{3}$ systems where application of an electric field during cooling below the Néel temperature has the effect of switching the interface magnetization and the exchange bias [346].

Ferrites, such as ferrimagnetic barium hexaferrite $\mathrm{BaFe}_{12} \mathrm{O}_{19}(\mathrm{BaM})$, can play an important role in the propagation of electromagnetic waves in microwave and millimeter wave devices (such as tunable filters, phase shifters, and circulators) due to their low losses in the gigahertz frequencies and high magnetic anisotropy [347]. Growing thick films and multilayers of ferrite/ferroelectric (such as $\mathrm{BaTiO}_{3}$ or PZT) compounds produces coupling of the magnetic response and ferroelectricity mediated by mechanical stress [348]. This leads to dual tunability of the permeability and permittivity by magnetic and electric fields [349]. Electric tunability of the ferromagnetic resonance (FMR) has been demonstrated in $\mathrm{BaM} / \mathrm{Ba}(\mathrm{Sr}) \mathrm{TiO}_{3}$ heterostructures [350].

\section{Interfaces with organic materials}

Organic materials are considered to be those made only of $\mathrm{H}, \mathrm{C}, \mathrm{O}$, and/or $\mathrm{N}$, while 'organic-based' materials can contain coordinating transition and/or rare earth atoms. Many organic materials are widely available and cheap, easy to make, low-weight and flexible. Therefore, organic materials are considered to be the key to mass produced, disposable electronics; but they are far from their maturity point. Recently, organic materials have been demonstrated in spintronics applications as barriers and/or electrodes in heterojunctions [19], but also may serve a greater purpose as spin injectors.

\subsection{Organic barriers}

Canonical organic materials are limited to the role of barriers in tunnel junctions, although GMR devices may also be possible with conducting polymers replacing the non-magnetic metals $(\mathrm{Cu})$. Dediu et al demonstrated $\mathrm{MR}$ at room temperature using the semiconductor sexithiophene $\left(\mathrm{T}_{6}\right)$ as a barrier between two LSMO electrodes [18]. Although weak, the response was definite and initiated a flurry of work toward hybrid-based junctions where the electrodes are inorganic highly spin-polarization solids (e.g. LSMO) while the insulating barriers are organic or metalorganic ultrathin layers. Xiong et al. used an $\mathrm{Alq}_{3}$ (tris(8-hydroxyquinoline) aluminum) spacer layer in a spin valve between LSMO and Co contacts [351]. Negative MR of $12 \%$ was obtained at low temperature. TMR was demonstrated in junctions prepared by placing single molecular layer of octanedithiol between two Ni contacts [352]. Santos et al showed spinpolarized tunneling through thin $\mathrm{Alq}_{3}$ barrier between Co and permalloy [17]. Xu et al observed TMR in LSMO and Co junctions where tetraphenyl porphyrin (TPP) and $\mathrm{Alq}_{3}$ were used as tunnel barrier [353]. In the case of organic tunnel junction barriers, surface roughness and decomposition at the organic insulator/inorganic metal interface has plagued much of this work. In particular, the techniques developed for thin film organic depositions in light emitting diodes have not matured to the point of forming reproducible, flat and ultrathin films at the precision needed in bulk tunneling devices. 


\subsection{Organic electrodes}

It is important to note that organic-based magnetic solids with finite spin polarization do exist but are rare, especially with ordering temperatures above room temperature. An implication of this rarity is that organic-based magnets are considered somewhat taboo by the traditional inorganic spintronics community because of high temperature decomposition and atmospheric sensitivity. Organic magnets are complexes, usually built from molecules, although nonmolecular solids do exist [24,354]. Systems involving single molecular magnets are not the same as those we highlight here [355].

The first organic-based magnets were discovered in the late 1950's, although the community was not fully entrenched until the mid to late 1980's where Oliver Kahn set the theoretical basis for organic exchange [24] and Miller and Epstein the experimental foundation [356]. Since this time, an energized group of mostly solid state chemists has charged ahead using their combined metal-organic synthetic and analytical training to achieve control and use of the $p$ orbital in addition to the canonical direct exchange from $d$ or $f$ orbitals; it should be noted that superexchange discussed for inorganic solids such as oxygen in $\mathrm{NiO}$ or the perovskite is very different from the molecular based exchange represented here where control of the ligand field is allowable.

There are two accepted organic-based systems which exhibit a remnant moment above room temperature: $\mathrm{V}[\mathrm{TCNE}]^{-}$first reported by Miller and Epstein [357] and $\mathrm{V}\left[\mathrm{Cr}\left(\mathrm{CN}_{6}\right)\right] \cdot \mathrm{H}_{2} 0$, also known as the prussian blue analog, by the Verdauger group [358]. Both systems exhibit antiferromagnetic exchange resulting in ferrimagnetism. On a related note, this lack of metallic state has subsequently led to the prediction by Prigodin and Epstein that a half-semiconductor character is possible from organic based magnets [359$363,25]$.

In organic-based solids, the challenge is to use organic ligands as the mediators of exchange, relative to most inorganic solids which utilize direct overlap due to the less than $3 \AA$ proximity of the metals. This means that the exchange energy can be, and usually is, considerably less for organic-based solids, implying that the temperature required to overcome exchange is also quite less. That is, with low Debye temperatures and weak exchange energy, electron-phonon and electronmagnon coupling easily destroy long range order in organic based solids. Despite this sensitivity, organicbased solids have still prevailed in demonstrating magnetoresistance response due to spin-polarized transport.

For the $\mathrm{V}[\mathrm{TCNE}]^{-}$system, thin film deposition [364] and spin-polarized transport has been reported $[25,359,360]$. In related systems, namely, in $\left[\mathrm{Fe}^{\mathrm{II}}\left(\mathrm{TCNE}^{-}\right)(\mathrm{NCMe})_{2}\right]^{+}\left[\mathrm{Fe}^{\mathrm{III}} \mathrm{Cl}_{4}\right]^{-}$, recent spin-polarized photoemission has provided direct demonstration that high polarization is indeed available near the Fermi edge. Ab initio calculations on other unpaired radical based systems [365-367] further back up high spin polarization, going as far as claiming half-metallic character.

The important points regarding interfaces, to consider particularly for organic-based magnets relevant to magnetoelectronic applications, are threefold: (1) the difference in free energy between the bulk and surface or interface of two organic-based materials is in general lower than that for fully inorganic or hybrid systems; (2) for those complexes which contain transition or rare-earth metals, the metal atoms are held in place by coordinating ligands, such that the problems associated with phase separation or agglomeration, as occur blatantly in dilute magnetic semiconductors [368], do not occur; (3) even if defects are formed at a hybrid or all organic-based interface, scattering of spin-polarized carriers is low relative to fully inorganic interfaces because of the reduced hyperfine and spin-orbit coupling of low $\mathrm{Z}$ and ${ }^{12} \mathrm{C}$ inactive atoms [22,23,369]. Possibly the most important future contribution of organic-based magnets will be their application in systems where spin-polarized semiconductors are required.

\section{Polar interfaces}

Nanoscale heterostructures have found very important applications in magnetic recording and memory applications. However, the ultimate goal is the replacement of the transistor. In that respect, oxidebased superlattices with polar interfaces have shown interesting properties. In particular, it was demonstrated experimentally that polar discontinuities cause the interface between two common wide-gap insulating oxides $\mathrm{LaAlO}_{3}$ (or $\mathrm{LaTiO}_{3}$ ) and $\mathrm{SrTiO}_{3}$ to become conducting [370,371]. The quasi-two-dimensional electron gas (2DEG) forming at the interface has extremely high carrier mobility and an electron density that is larger by an order of magnitude than the density 
of 2DEG at known semiconductor interfaces. This phenomenon is interesting due to possible application in all-oxide ultra-small, high-electron mobility, field-effect transistors [372]. Very recently, evidence of magnetism at the interface between these non-magnetic oxides was found, which may open exciting perspectives beyond transistor functionality [373].

Polar surfaces have not been of much practical interest because polar discontinuities introduce vary large energy cost for atomically abrupt surfaces. As a result such surfaces are either not stable or they reconstruct. In case of polar interfaces, such reconstruction leads to disorder [374]. Mixed valence oxides, however, allow for the possibility of charge (rather than ion) redistribution, which opens the possibility for atomically abrupt polar interfaces. In particular, perovskite oxides exhibit a wide variety of valence states which can be described with the expression $\mathrm{A}^{\mathrm{X}+} \mathrm{B}^{\mathrm{Y}+} \mathrm{O}_{3}{ }^{2-}$, where $\mathrm{X}+\mathrm{Y}=6$. In the $(001)$ direction, the solid consists of alternating planes of $\mathrm{A}^{\mathrm{X}+} \mathrm{O}^{2-}$ and $\mathrm{B}^{\mathrm{Y}+} \mathrm{O}_{2}{ }^{2-}$ which can be charged. This is the case for $\mathrm{LaAlO}_{3} / \mathrm{SrTiO}_{3}(001)$ heterostructure, where $\mathrm{La}^{3+} \mathrm{Al}^{3+} \mathrm{O}_{3}{ }^{2-}$ consists of alternating $(\mathrm{LaO})^{+}$and $\left(\mathrm{AlO}_{2}\right)^{-}$ planes and $\mathrm{Sr}^{2+} \mathrm{Ti}^{4+} \mathrm{O}_{3}{ }^{2-}$ consists of alternating $(\mathrm{SrO})^{0}$ and $\left(\mathrm{TiO}_{2}\right)^{0}$ planes, leading to a polar interface according to the bulk valence. However, due to accessible mixed valence of $\mathrm{Ti}$, allowing for its reduction towards $\mathrm{Ti}^{3+}$, half an electron per twodimensional unit cell can be transferred across the interface to compensate for the electrostatic potential divergence. Thus, there are uncompensated $n$-type carriers at the $(\mathrm{LaO})^{+} /\left(\mathrm{TiO}_{2}\right)^{0}$ interface and $p$-type carriers at the $\left(\mathrm{AlO}_{2}\right)^{-} /(\mathrm{SrO})^{0}$ interface, which are supposed to make these interfaces conductive. The $n$ type conductance has indeed been confirmed experimentally for the $(\mathrm{LaO})^{+} /\left(\mathrm{TiO}_{2}\right)^{0}$ interface, but the $\left(\mathrm{AlO}_{2}\right)^{-} /(\mathrm{SrO})^{0}$ interface was found to be insulating [371,372].

First-principles calculations of $\mathrm{LaAlO}_{3} / \mathrm{SrTiO}_{3}$ and $\mathrm{LaTiO}_{3} / \mathrm{SrTiO}_{3}$ superlattices, based on density functional theory within the local density approximation (LDA) $[375,376]$ and the $\mathrm{LDA}+U$ approximation $[377,378]$, have been performed. Calculations essentially confirm the presence of charge carriers in both $n$-type ( $1 / 2$ electron on the interface Ti-3d band) and $p$-type interfaces $(1 / 2$ hole on the interface $\mathrm{O}-2 p)$. The lack of experimental evidence for conductance in the $p$ type interface is still an open question although disorder and $\mathrm{O}$ vacancies at the interface have been proposed. Another question is the dimension of the $2 \mathrm{DEG}$ layer.
Experimental techniques lack the resolution to measure the size of the conducting layer. Only very recently, conduction as a function of the distance from the interface was mapped by conducting-tip atomic force microscope [379]. The 2DEG was confirmed to be localized within a few nanometers from the interface. Calculations also predict localization within several unit cells of the interface at least in the presence of strong electron correlations [378,380].

Recently, it was demonstrated that the interface between the non-magnetic $\mathrm{LaAlO}_{3}$ and $\mathrm{SrTiO}_{3}$ becomes magnetic at low temperatures [373]. This was attributed to ferromagnetic alignment of the induced electrons on the Ti-3d conduction band. This behavior is corroborated by spin-polarized first principles calculations of $\mathrm{LaAlO}_{3} / \mathrm{SrTiO}_{3}$ interface [377], as well as $\mathrm{LaTiO}_{3} / \mathrm{SrTiO}_{3}$ interfaces [378]. First principles spinpolarized calculations reveal Stoner-type magnetism [380]. The interface of a $\left(\mathrm{LaAlO}_{3}\right)_{3} /\left(\mathrm{SrTiO}_{3}\right)_{3}$ superlattice is magnetic with magnetic moment on the $\mathrm{Ti}^{3+}$ atom of $0.2 \mu \mathrm{B}$. For thicker $\mathrm{SrTiO}_{3}$ layers the magnetism decreases and eventually disappears because the electron gas spreads over more than one unit cell making the electron delocalized across the superlattice and violating the Stoner criterion for magnetism. Thus, the interface magnetization in these superlattices is due to geometric confinement of the electron gas. The inclusion of electron correlations via the $\mathrm{LDA}+U$ approximation with $U=5 \mathrm{eV}$ on $\mathrm{Ti}$ atoms makes the twodimensional electron gas more localized and halfmetallic strengthening the interface magnetization [380].

\section{Conclusions}

It is now increasingly clear that the physics of heterostructures is as much dominated by the interfaces as by the bulk materials. Modern experimental techniques allow the deposition of uniform thin films consisting only of a few atomic layers and this opens an avenue to the construction of artificial layered heterostructures made of nanoscale size slabs of different materials. Such materials have a very high ratio of interfaces to bulk material, which causes their properties to be increasingly determined by the properties of the interfaces. This leads to a number of complications but also to a wealth of opportunities to tailor desired properties.

Thus, looking at bulk materials properties alone is no longer sufficient for understanding heterostructure 
properties: band structure is key to many phenomena. Band structure matching is utilized in spin valves where the interface between two metals leads to preferential transmission of one of the spin channels when the magnetizations are aligned. This is the origin of the GMR effect. Similarly, complex band structure matching is used in magnetic tunnel junctions where matching propagating states in the electrodes to the complex bands of the barrier leads to lower rate of decay for one of the spin channels when magnetizations are aligned. This is the origin of the TMR effect. Extreme cases of band structure matching might occur at the interfaces with half-metallic materials, but such interfaces are often dominated by spin minority states, that often occur at interfaces with nominally half metallic materials.

Other examples of band structure matching are spin injection and spin filtering applications, the purpose of which is to inject highly spin-polarized current from a ferromagnet to a semiconductor. The ferromagnet/ insulator interface, in addition to preserving the intrinsic spin polarization of the ferromagnet, also serves to match the resistance of the metal to that of the semiconductor. This is crucial for achieving a high degree of spin polarization, since the resistance of the ferromagnet/semiconductor interface is dominated by the nonmagnetic semiconductor. Magnetic insulators have different barrier heights for the different spin channels, thus enhancing the inherent spin polarization of the ferromagnetic electrode.

Matching of different ferroic orders, such as ferroelectric/ferromagnetic, has emerged as a way to make multifunctional materials i.e. materials which can be controlled or modulated by the application of several different stimuli, such as by both applied magnetic and electric fields. Combinations of ferromagnetic and ferroelectric materials may also exhibit phenomena, such as the magnetoelectric effect, which are present in bulk materials but are rare. This suggests a major role for not only magnetic moments and the spin polarization but also electric dipoles at device interfaces.

Electrostatic matching is especially pronounced at polar interfaces. Polar surfaces and interfaces are energetically expensive and are known to reconstruct (surfaces) or to be rough (interfaces). However, it has been demonstrated that in some cases a charge compensation of the electrostatic discontinuity can produce two-dimensional electron gas localized close to the interface. This electron channel is much narrower than that at semiconductor interaces and the mobility of the electron gas much higher. This phenomenon can have applications in the next generation field effect transistors.

Organic-inorganic interface matching is especially important not only for the purpose of cheap electronics but also for biological applications in which traditional electronics are interfaced to living organisms.

Finally, the important point to note is that nanoscale heterojunctions present a different paradigm of 'interface science' in which the properties are essentially determined by the interface between traditional materials: as such, this is an important playground for surface science where fundamentally new phenomena may be observed.

\section{Acknowledgements}

This work was supported by the National Science Foundation through Materials Research Science and Engineering Center at University of Nebraska-Lincoln (grant MRSEC DMR-0213808), the Office of Naval Research (ONR Grant No. N00014-06-1-0616), the Nebraska Research Initiative, and the Nanoelectronics Research Initiative. S.J.J. is grateful to The Royal Society for a University Research Fellowship. J.P.V. thanks K.D. Belashchenko and L.J. Brillson for the helpful discussions. 


\section{References}

1. E.W. Plummer, Ismail, R. Matzdorf, A.V. Melechko and J. Zhang, Prog. Surf. Sci. 67 (2001) 17.

2. F.J. Himpsel, J.E. Ortega, G.J. Mankey, R.F. Willis, Adv. Phys. 47 (1998) 511.

3. T.C. Chiang, Surf. Sci. Rep. 39 (2000) 181.

4. F. J. Himpsel, J. Phys.: Cond. Mat. 11 (1999) 9483.

5. S.A. Wolf, D.D. Awschalom, R.A. Buhrman, J.M. Daughton, S. von Molnár, M.L. Roukes, A.Y. Chtchelkanova, D.M. Treger, Science 294 (2001) 1488.

6. I. Zutic, J. Fabian, and S. Das Sarma, Rev. Mod. Phys. 76 (2004) 323.

7. C. Chappert, A. Fert, and F.N. Van Dau, Nature Mat. 6 (2007) 813.

8. M.L. Plumer, J. van Ek, D. Weller (Eds), The Physics of Ultrahigh-Density Magnetic Recording, Springer Series in Surface Sciences 41 (2001) 352.

9. S. Tehrani et al., Proc. IEEE 91 (2003) 703.

10. W.J. Gallagher and S.S.P. Parkin, IBM J. Res. Dev. 50 (2006) 5.

11. D.D. Awschalom, M.E. Flatté, Nature Phys. 3 (2007) 153.

12. W.F. Egelhoff, M.D. Stiles, D.P. Pappas, D.T. Pierce, J.M. Byers, M.B. Johnson, B.T. Jonker, S.F. Alvarado, J.F. Gregg, J.A.C. Bland, and R.A. Buhrman, Science 296 (2002) 1195.

13. R. Jansen, Nature Phys. 3 (2007) 521.

14. H. Ohno, Science 281 (1998) 951.

15. J.M.D. Coey, Curr. Opinion Sol. St. \& Mater. Sci. 10 (2006) 83.

16. S.A. Chambers, T.C. Droubay, C.M. Wang, K.M. Rosso, S.M. Heald, D.A. Schwartz, K.R. Kittilstved, D.R. Gamelin, Mater. Today 9 (2006) 28.

17. T.S. Santos, J.S. Lee, P. Migdal, I.C. Lekshmi, B. Satpati, and J. S. Moodera, Phys. Rev. Lett. 98 (2007) 016601.

18. V. Dediu, M. Murgia, F.C. Matacotta, C. Taliani, and S. Barbanera, Sol. St. Comm. 122 (2002) 181.

19. W.J.M. Naber, S. Faez, and W.G. van der Wiel, J. Phys. D: Appl. Phys. 40 (2007) R205.

20. C. Jachim, J. Gimzewski, and A. Aviram, Nature 408 (2000) 541.

21. J. Heath and M. Ratner, Phys. Today 5 (2003) 43.

22. A.R. Rocha, V.M. Garcia-Suarez, S.W. Bailey, C.J. Lambert, J. Ferrer, S. Sanvito, Nature Mater. 4 (2005) 335.

23. S. Sanvito and A.R. Rocha, J. Comp. Theor. Nanosci. 3 (2006) 624.

24. O. Kahn, Molecular Magnetism, John Wiley \& Sons, Singapore (1993).

25. A.J. Epstein, MRS Bulletin 28 (2003) 492.

26. S. Sahoo, T. Kontos, J. Furer, C. Hoffmann, M. Gräber, A. Cottet, and C. Schönenberger, Nature Phys. 1 (2005) 99.
27. J.-P. Cleuziou, W. Wernsdorfer, V. Bouchiat, T. Ondarçuhu, M. Monthioux, Nature Nanotech. 1 (2006) 53.

28. N. Tombros, C. Jozsa, M. Popinciuc, H.T. Jonkman, B.J. van Wees, Nature 448 (2007) 571.

29. V.M. Karpan, G. Giovannetti, P.A. Khomyakov, M. Talanana, A.A. Starikov, M. Zwierzycki, J. van den Brink, G. Brocks, and P.J. Kelly, Phys. Rev. Lett. 99 (2007) 176602.

30. M. Dawber, K.M. Rabe, and J.F. Scott, Rev. Mod. Phys. 77 (2005) 1083.

31. J.F. Scott, J. Phys.: Cond. Matt. 18 (2006) R361.

32. M. Fiebig, J. Phys. D 38 (2005) R123.

33. W. Eerenstein, N.D. Mathur, and J.F. Scott, Nature 442 (2006) 759.

34. R. Ramesh and N.A. Spaldin, Nature Materials 6 (2007) 21.

35. N.A. Hill, J. Phys. Chem. B 104 (2000) 6694.

36. T. Zhao, et al., Nature Mater. 5 (2006) 823.

37. F. Zavaliche, et al., Nano Lett. 7 (2007) 1586.

38. N.F. Mott, Proc. R. Soc. London, Ser. A 153 (1936) 699; 156 (1936) 368.

39. P.M. Tedrow and R. Meservey, Phys. Rev. Lett. 26 (1971) 192; Phys. Rev. B 7 (1973) 318.

40. M. Jullière, Phys. Lett. A 54 (1975) 225.

41. P. Grünberg, R. Schreiber, Y. Pang, M. B. Brodsky, and H. Sowers, Phys. Rev. Lett. 57 (1986) 2442.

42. S.S.P. Parkin, N. More, and K. Roche, Phys. Rev. Lett. 64 (1990) 2304.

43. J. Faure-Vincent, C. Tiusan, C. Bellouard, E. Popova, M. Hehn, F. Montaigne, and A. Schuhl, Phys. Rev. Lett. 89 (2002) 107206.

44. R.R. Gareev, L.L. Pohlmann, S. Stein, D.E. Bürgler, P.A. Grünberg, and M. Siegel, J. Appl. Phys. 93 (2003) 8038.

45. T. Katayama, S. Yuasa, J.P. Velev, M.Ye. Zhuravlev, S.S. Jaswal, and E.Y. Tsymbal, Appl. Phys. Lett. 89 (2006) 112503.

46. J.C. Slonczewski, Phys. Rev. 39 (1989) 6995.

47. R.P. Erickson, K.B. Hathaway, and J.R. Cullen, Phys. Rev. B 47 (1993) 2626.

48. M.Ye. Zhuravlev, E.Y. Tsymbal, and A.V. Vedyayev , Phys. Rev. Lett. 94 (2005) 026806.

49. P. Bruno, J. Magn. Magn. Mater. 121 (1993) 248; Phys. Rev. B 52 (1995) 411.

50. M. Stiles, Phys. Rev. B 48 (1993) 7238.

51. M.N. Baibich, J.M. Broto, A. Fert, F.N. Van Dau, F. Petroff, P. Etienne, G. Creuzet, A. Friederich, and J. Chazelas, Phys. Rev. Lett. 61 (1988) 2472.

52. G. Binasch, P. Grunberg, F. Saurenbach, and W. Zinn, Phys. Rev. B 39 (1989) 4828.

53. A.Barthelemy, A.Fert, and F.Petroff, in Handbook of Magnetic Materials, edited by K.H.J.Buschow (Amsterdam: Elsevier, 1999) 1.

54. P.M. Levy, Sol. State Phys. 47 (1994) 367. 
55. M. Gijs and G. Bauer, Adv. Phys. 46 (1997) 285.

56. J. Bass and W. P. Pratt, J. Magn. Magn. Mater. 200 (1999) 274.

57. U. Hartmann (Ed.), Magnetic Multilayers and Giant Magnetoresistance: Fundamentals and Industrial Applications, Springer (2000).

58. E.Y. Tsymbal and D.G. Pettifor, Sol. State Phys. 56 (2001) 113.

59. I. Mertig and P. Levy, in Spin-dependent Transport in Magnetic Nanostructures, Ed. by S. Maekawa, Gordon and Breach Publishing Group (2002).

60. J. Bass and W.P. Pratt, J. Phys.: Cond. Matt. 19 (2007) 183201.

61. T.R. McGuire and R.I. Potter, IEEE Trans. Magn. 11 (1975) 1018.

62. B. Dieny, V.S. Speriosu, S.S. Parkin, B.A. Gurney, D. R. Wilhoit, and D. Mauri, Phys. Rev. B 43 (1991) R1297.

63. J.S. Moodera, L.R. Kinder, T.M. Wong, and R. Meservey, Phys. Rev. Lett. 74 (1995) 3273.

64. D. Wang et al., IEEE Trans. Magn. 40 (2004) 2269.

65. S.S.P. Parkin, C. Kaiser, A. Panchula, P.M. Rice, B. Hughes, M. Samant and S.-H. Yang, Nature Mater. 3 (2004) 862.

66. S. Yuasa, T. Nagahama, A. Fukushima, Y. Suzuki and K. Ando, Nature Mater. 3 (2004) 868.

67. W.H. Butler, X.-G. Zhang, T.C. Schulthess, and J.M. MacLaren, Phys. Rev. B 63 (2001) 054416.

68. J. Mathon and A. Umerski, Phys. Rev. B 63 (2001) R220403.

69. R. Meservey and P.M. Tedrow, Phys. Rep. 238 (1994) 173.

70. J.S. Moodera, J. Nassar, and G. Mathon, Ann. Rev. Mater. Sci. 29 (1999) 381.

71. E.Y. Tsymbal, O.N. Mryasov, and P.R. LeClair, J. Phys.: Cond. Mat. 15 (2003) R109.

72. E.Y. Tsymbal, in Handbook of Magnetism and Advanced Magnetic Materials 5, Eds. H. Krohnmüller and S.S.P. Parkin (Chichester, UK: John Wiley \& Sons Ltd., 2006) 2963.

73. X.-G. Zhang and W.H. Butler, J. Phys.: Cond. Mat. 15 (2003) R1603.

74. R.A. de Groot, F.M. Mueller, P.G. van Engen, and K.H. J. Buschow, Phys. Rev. Lett. 50 (1983) 2024.

75. I. Galankis and P.H. Dederichs (Eds.), Lecture Notes in Physics 676, Springer (2005).

76. P.A. Dowben (Ed.), J. Phys.: Cond. Mat. 19 (2007).

77. M. Bibes and A. Barthélémy, IEEE Trans. El. Dev. 54 (2007) 1003.

78. E.Y. Tsymbal and H. Kohlstedt, Science 313 (2006) 181.

79. M.Ye. Zhuravlev, R.F. Sabirianov, S.S. Jaswal, and E.Y. Tsymbal, Phys. Rev. Lett. 94 (2005) 246802.

80. H. Kohlstedt, N.A. Pertsev, J. Rodríguez Contreras, and R. Waser, Phys. Rev. B 72 (2005) 125341.
81. J. Rodriguez Contreras et al., Appl. Phys. Lett. 83 (2003) 4595.

82. M. Gajek, M. Bibes, S. Fusil, K. Bouzehouane, J. Fontcuberta, A. Barthélémy, and A. Fert, Nature Mater. 6 (2007) 296.

83. G. Schmidt, J. Phys. D: Appl. Phys. 38 (2005) R107.

84. G. Schmidt, D. Ferrand, L.W. Molenkamp, A.T. Filip and B. J. van Wees, Phys. Rev. B 62 (2000) R4790.

85. E.I. Rashba, Phys. Rev. B 62 (2001) R16267.

86. T.S. Santos and J.S. Moodera, Phys. Rev. B 69 (2004) R241203.

87. U. Lüders, A. Barthelemy, M. Bibes, K. Bouzehouane, S. Fusil, E. Jacquet, J.-P. Contour, J.-F. Bobo, J. Fontcubeerta, and A. Fert, Adv. Mater. 18 (2006) 1733.

88. H. Lüth, Surfaces and interfaces in solids, Springer (1993); Solid surfaces, interfaces, and thin films, Springer (2001).

89. F. Flores and C. Tejedor, J. Phys. C 20 (1987) 147.

90. L.J. Brillson, in Contacts to Semiconductors: Fundamentals and Technology, ed. L. J. Brillson (Noyes, Park Ridge, NJ, 1993) 333.

91. L.J. Brillson, Surf. Sci. Rep. 2 (1982) 123; Surf. Sci. 299/300 (1994) 909.

92. R.T. Tung, Phys. Rev. Lett. 84 (2000) 6078.

93. J. Frenkel, Phys. Rev. 36 (1930) 1604.

94. I. Giaever, Phys. Rev. Lett. 5 (1960) 147; ibid. (1960) 464.

95. R. Landauer, IBM J. Res. and Dev. 1 (1957) 223; Z. Physik B 68 (1987) 217; J. Math. Phys. 37 (1996) 5259.

96. M. Büttiker, Y. Imry, R. Landauer, and S. Pinhas, Phys. Rev. B 31 (1985) 6207.

97. S. Datta, Electronic Transport in Mesoscopic Systems, Cambridge University Press (1997).

98. J. Velev and W. Butler, Phys. Rev. 69 (2004) 024404.

99. J. Kudrnovsky, V. Drchal, C. Blaas, P. Weinberger, I. Turek, and P. Bruno, Phys. Rev. B 62 (2000) 15084; K. Xia, P. J. Kelly, G.E.W. Bauer, I. Turek, J. Kudrnovsky, and V. Drchal, ibid. 63 (2001) 064407; K. Xia, M. Zwierzycki, M. Talanana, P. J. Kelly, and G.E.W. Bauer, Phys. Rev. B 73 (2006) 064420.

100. J. Velev and W. Butler, J. Appl. Phys. 97 (2005) $10 \mathrm{C} 517$.

101. V. Drchal, J. Kudrnovsky, P. Bruno, P.H. Dederichs, I. Turek, and P. Weinberger, Phys. Rev. B 65 (2002) 214414.

102. D. Wortmann, H. Ishida, and S. Blugel, Phys. Rev. B 72 (2005) 235113.

103. Y. Sharvin, Zh. Eksp. Teor. Phys. 48 (1965) 984; Sov. Phys. JETP 21 (1965) 655.

104. K. Schep, P. Kelly, and G. Bauer, Phys. Rev. Lett. 74 (1995) 586; Phys. Rev. B 57 (1998) 8907.

105. I.I. Mazin, Phys. Rev. Lett. 83 (1999) 1427; Europhys. Lett. 55 (2001) 404.

106. A. Fert, J. Phys. C: Solid St. Phys. 2 (1969) 1784.

107. J.W.F. Dorleijn, in Philips Res.Rep. 31 (1976) 287. 
108. I.A. Campbell and A. Fert, in Ferromagnetic Materials, edited by E.P. Wohlfarth (Amsterdam: North Holland, 1982) 769.

109. I. Mertig, Rep.Prog.Phys, 62 (1999) 237.

110. E.Y. Tsymbal and D.G. Pettifor, J. Magn. Magn. Mater. 202 (1999) 163.

111. G. Deutscher, Rev. Mod. Phys. 77 (2005) 109.

112. M.B. Stearns, J. Magn. Magn. Mat. 5 (1977) 167.

113. K.D. Belashchenko, E.Y. Tsymbal, M. van Schilfgaarde, D.A. Stewart, I.I. Oleynik, and S. S. Jaswal, Phys. Rev. B 69 (2004) 174408.

114. Y.-C. Chang and J. Schulman, Phys. Rev. B 25 (1982) 3975.

115. J. Velev and W. Butler, J. Phys.: Cond. Matter 16 (2004) R637 and references therein.

116. V. Heine, Proc. Phys. Soc. 81 (1963) 300.

117. G.C. Osbourn and D.L. Smith, Phys. Rev. B 19 (1979) 2124.

118. E.Y. Tsymbal and K.D. Belashchenko, J. Appl. Phys. 97 (2005) 10C910.

119. E.Y. Tsymbal, K.D. Belashchenko, J.P. Velev, S.S. Jaswal, M. van Schilfgaarde, I.I. Oleynik, and D.A. Stewart, Prog. Mater. Sci. 52 (2007) 401.

120. T. Ando, Phys. Rev. B 44 (1991) 8017.

121. G. Brocks, V.M. Karpan, P.J. Kelly, P.A. Khomyakov, I. Marushchenko, A. Starikov, M. Talanana, I. Turek, K. Xia, P.X. Xu, M. Zwierzycki, G.E.W. Bauer, $\psi_{k}$ Newslett. (2007) 144.

122. Ph. Mavropoulos, N. Papanikolaou, and P.H. Dederichs,. Phys. Rev. Lett. 85 (2000) 1088.

123. J.P. Velev, K.D. Belashchenko, D.A. Stewart, M. van Schilfgaarde, S.S. Jaswal, and E.Y. Tsymbal, Phys. Rev. Lett. 95 (2005) 216601.

124. J.M. de Teresa et al., Phys. Rev. Lett. 82 (1999) 4288; Science 286 (1999) 507.

125. M. Bibes et al., Appl. Phys. Lett. 82 (2003) 3269.

126. V. Garcia et al., Appl. Phys. Lett. 87 (2005) 212501.

127. J.P. Velev and K.D. Belashchenko, unpublished.

128. Ya.B. Losovyj, I.N. Yakovkin, S.D. Barrett, T. Komesu, and P.A. Dowben, Surf. Sci. 520 (2002) 43.

129. Ya.B. Losovyj, S.M. Zuber, and P.A. Dowben, Vacuum 74 (2004) 147.

130. I.E. Tamm, Phys. Z. Soviet Union 1 (1932) 732.

131. A.W. Maue, Z. Physik 94 (1935) 1.

132. E.T. Goodwin, Proc. Camb. Phil. Soc. 35 (1939) 205.

133. W. Shockley, Phys. Rev. 56 (1939) 317.

134. W. Schottky, Z. Phys. 113 (1939) 367.

135. N.F. Mott, Proc. Roy. Soc. A 171 (1939) 27.

136. J. Bardeen, Phys. Rev. 71 (1947) 717.

137. V. Heine, Phys. Rev. 138 (1965) A1689.

138. R.T. Tung, Phys. Rev. B 64 (2001) 205310.

139. O. Wunnicke, N. Papanikolaou, R. Zeller, P. Dederichs, V. Drchal, and J. Kudrnovsky, Phys. Rev. B 65 (2002) 064425.

140.A.M. Bratkovsky, Phys. Rev. B 56 (1997) 2344.
141. A. N. Chantis, K. D. Belashchenko, E. Y. Tsymbal, and M. van Schilfgaarde, Phys. Rev. Lett. 98 (2007) 046601 .

142. L. Gao, X. Jiang, S. Yang, J. D. Burton, E. Y. Tsymbal, and S. S. P. Parkin, Phys. Rev. Lett. 99 (2007) 226602.

143. J. D. Burton, R. F. Sabirianov, J. P. Velev, O. N. Mryasov, and E. Y. Tsymbal, Phys. Rev. B 76, 144430 (2007).

144. M.F. Crommie, C.P. Lutz, and D.M. Eigler, Science 262 (1993) 218; Nature 363 (1993) 524.

145. E.J. Heller, M.F. Crommie, C.P. Lutz, and D.M. Eigler, Nature 369 (1994) 464.

146. H.C. Manorahan, C.P. Lutz, and D.M. Eigler, Nature 403 (2000) 512.

147. L. Petersen, P. Laitenberger, E. Laegsgaard, and F. Besenbacher, Phys. Rev. B 58 (1998) 7361.

148. P. Avouris, I.W. Lyo and P. Molinasmata, Chem. Phys. Lett. 240 (1995) 423.

149. P. Avouris, I.W. Lyo, R.E. Walkup and Y. Hasegawa, J. Vac. Sci. Technol. B 12 (1994) 1447.

150. Y. Hasegawa and P. Avouris, Phys. Rev. Lett. 71 (1993) 1071.

151. P. Avouris and I. W. Lyo, Science 264 (1994) 942.

152. W. Chen, V. Madhavan, T. Jamneala and M.F. Crommie, Phys. Rev. Lett. 80 (1998) 1469.

153. M.F. Crommie, J. El. Spect. Rel. Phenom. 109 (2000) 1.

154. P.T. Sprunger, L. Petersen, E.W. Plummer, E. Laegsgaard and F. Besenbacher, Science 275 (1997) 1764.

155. P. Hofmann, B.G. Briner, M. Doering, H.P. Rust, E.W. Plummer, and A.M. Bradshaw, Phys. Rev. Lett. 79 (1997) 265.

156. B.G. Briner, P. Hofmann, M. Doering, H.P. Rust, E.W. Plummer, and A.M. Bradshaw, Phys. Rev. B 58 (1998) 13931.

157. L. Petersen, B. Schaeffer, E. Laegsgaard, I. Stensgaard, and F. Besenbacher, Surf. Sci. 457 (2000) 319.

158. K.H. Hansen, J. Gottschalck, L. Petersen, B. Hammer, E. Laegsgaard, F. Besenbacher, and I. Stensgaard, Phys. Rev. B 63 (2001) 115421.

159. P.A. Dowben and S.J. Jenkins, in Finite-Temperature Half-Metallic Ferromagnets, edited by Anant Narlikar, Springer Verlag (2005) 295.

160. S. Lee, J. Mazurowski, G. Ramseyer, and P.A. Dowben, J. Appl. Phys. 72 (1992) 4925.

161. D.N. McIlroy, S.-D. Hwang, K. Yang, N. Remmes, P.A. Dowben, A.A. Ahmad, N.J. Ianno, J.Z. Li, J.Y. Lin, and H.X. Jiang, Appl. Phys. A 67 (1998) 335.

162. L. Bernard, J. Monson, A. Sokolov, Zong-Yuan Liu, C.S. Yang, P.A. Dowben, B. Doudin, A. Harken, P. Welsch and B.W. Robertson, Appl. Phys. Lett. 83 (2003) 3743.

163. T. Greber, W. Auwärter and J. Ostwalder, in The Physics of Low Dimensional Systems, edited by J.L. Moran-Lopez, Plenum, New York (2000). 
164. J. Osterwalder, J. El. Spectr. Rel. Phenom. 117-118 (2001) 71.

165. J. Mathon, Phys. Rev. B 56 (1997) 11810.

166. R.A. Lukaszew, Y. Sheng, C. Uher and R. Clarke, Appl. Phys. Lett. 75 (1999) 1941.

167. S. Ishida, S. Fujii, S. Kawhiwagi, and S. Asano, J. Phys. Soc. Jpn. 64 (1995) 2152.

168. K. Schwarz, J. Phys. F: Met. Phys. 16 (1986) L211.

169. S.F. Alvarado, M. Erbudak, and P. Munz, Phys. Rev. B 14 (1976) 2740.

170. A. Yanase and K. Siratori, J. Phys. Soc. Jpn. 53 (1984) 312.

171. Y. Okimoto, K. Katsufuji, T. Ishikawa, A. Urushibara, T. Arima, and Y. Tokura, Phys. Rev. Lett. 75 (1995) 109.

172. J.Y.T. Wei, N.-C. Yeh, and R.P. Vasques, Phys. Rev. Lett. 79 (1997) 5150.

173. I.I. Mazin, Appl. Phys. Lett. 77 (2000) 3000.

174. J.H. Park, E. Vescovo, H.J. Kim, C. Kwon, R. Ramesh, and T. Venkatesan, Nature 392 (1998) 794.

175. R. Bertacco, M. Portalupi, M. Marcon, L. Duo, F. Ciccacci, M. Bowen, J.P. Contour and A. Barthélémy, J. Magn. Magn. Mat. 242 (2002) 710.

176. D. Ristoiu, J. P. Nozières, C. N. Borca, T. Komesu, H.K. Jeong, and P. A. Dowben, Europhys. Lett. 49 (2000) 624.

177. C.N. Borca, T. Komesu, H.-K. Jeong, P.A. Dowben, D. Ristoiu, C. Hordequin, J.P. Nozières, J. Pierre, S. Stadler and Y.U. Idzerda, Phys. Rev. B 64 (2001) 052409 .

178. Y. Ji, G. J. Strijkers, F.Y. Yang, C.L. Chien, J.M. Byers, A. Anguelouch, G. Xiao, and A. Gupta, Phys. Rev. Lett. 86 (2001) 5585.

179. J.S. Parker, S.M. Watts, P.G. Ivanov, and P. Xiong, Phys. Rev. Lett. 88 (2002) 196601.

180. R.J. Soulen, J.M. Byers, B. Nadgormy, T. Ambrose, S.F. Cheng, P.R. Broussard, C.T. Tanaka, J. Nowak, J.S. Moodera, A. Barry, and J.M.D. Coey, Science 282 (1998) 85.

181. K.P. Kämper, W. Schmitt, G. Güntherodt, R.J. Gambino, and R. Ruf, Phys. Rev. Lett. 59 (1987) 2788.

182. Y.S. Dedkov, M. Fonine, C. Konig, U. Rüdiger, G. Güntherodt, S. Senz, and D. Hesse, Appl. Phys. Lett. 80 (2002) 4181 .

183. R. Skomski and P.A. Dowben, Europhys. Lett. 58 (2002) 544.

184. P.A. Dowben and R. Skomski, J. Appl. Phys. 93 (2003) 7948.

185. P.A. Dowben and R. Skomski, J. Appl. Phys. 95 (2004) 7453.

186. R. Skomski, J. Zhou, P.A. Dowben and D.J. Sellmyer, J. Appl. Phys. 97 (2005) 10C305.

187. R. Skomski, J. Phys. Cond. Matt. 19 (2007) 315202.

188. D.M. Edwars and J.A. Hertz, J. Phys. F: Met. Phys. 3 (1973) 2191.
189. V.Y. Irkhin and M.I. Katsnelson, Fiz. Tverd. Tela 25 (1983) 3383.

190. V.Y. Irkhin and M.I. Katsnelson, J. Phys. C. Sol. St. Phys. 18 (1985) 4173.

191. V.Y. Irkhin and M.I. Katsnelson, Phys. Rev. B 73 (2006) 104426.

192. V.Y. Irkhin, M.I. Katsnelson, A.I. Lichtenstein, J. Phys. Cond. Mat. 19 (2007) 315201.

193. A.H. MacDonald, T. Jungwirth and M. Kasner, Phys. Rev. Lett. 81 (1998) 705.

194. H. Itoh, T. Ohsawa, and J. Inoue, Phys. Rev. Lett. 84 (2000) 2501.

195. C.M. Fang, G.A. de Wijs, R.A. de Groot, J. Appl. Phys. 91 (2002) 8340.

196. I. Galanakis, J. Phys. Cond. Matt. 14 (2002) 6329.

197. W.H. Xie, B.G. Liu, J. Phys.: Cond. Matt. 15 (2003) 5085.

198. S. Picozzi, A. Continenza, A.J. Freeman, J. Appl. Phys. 94 (2003) 4723.

199. S. J. Jenkins and D.A. King, Surf. Sci. 501 (2002) L185.

200. S. Picozzi, A. Continenza, A.J. Freeman, J. Phys. Chem. Sol. 64 (2003) 1697.

201. V.K. Lazarov, Ph.D. Thesis, University of WisconsinMilwaukee (2003).

202. S. J. Jenkins and D.A. King, Surf. Sci. 494 (2001) L793.

203. P. Mavropoulos, M. Lezaic, and S. Blugel, Phys. Rev. B 72 (2005) 174428.

204. G.A. de Wijs and R.A. de Groot, Phys. Rev. B 64 (2001) 020402.

205. W.E. Pickett and J.S. Moodera, Phys. Today 5 (2001) 39.

206. Y. Lu, X.W. Li, G.Q. Gong, G. Xiao, A. Gupta, P. Lecoeur, J.Z. Sun, Y.Y. Wang, and V.P. Dravid, Phys. Rev. B 54 (1996) R8357.

207. M. Viret, M. Drouet, J. Nassar, J.P. Contour, C. Fermon, and A. Fert, Europhys. Lett. 39 (1997) 545.

208. J.Z. Sun, Physica C 350 (2001) 215.

209. D.C. Worledge and T.H. Geballe, Appl. Phys. Lett. 76 (2000) 900.

210. M. Bowen, M. Bibes, A. Barthelemy, et al., Appl. Phys. Lett. 82 (2003) 233.

211. M. Bowen, A. Barthelemy, M. Bibes, E. Jacquet, J.P. Contour, A. Fert, D. Wortmann and S. Blügel, J. Phys. Cond. Matter 17 (2005) L407.

212. M. Bowen, A. Barthélémy, M. Bibes, E. Jacquet, J.-P. Contour, F. Ciccacci, L. Duo and R. Bertacco Phys. Rev. Lett 95 (2005) 137203.

213. B. Nadgorny, J. Phys. Cond. Matter 19 (2007) 315209.

214. B. Nadgorny, I.I. Mazin, M. Osofsky, R.J. Soulen, P. Broussard, R.M. Stroud, D.J. Singh, V.G. Harris, A. Aresenov and Ya. Mukovskii, Phys. Rev. B 63 (2001) 184433.

215. H. Yamada et al, Science 305 (2004) 646.

216. M. Ziese, Rep. Prog. Phys. 65 (2002) 143. 
217. C.A. Ventrice, D.R. Borst, H. Geisler, J. van Ek, Y.B. Losovyj, P.S. Robbert, U. Diebold, J.A. Rodriguez, G.X. Miao and A. Gupta, J. Phys. Cond. Mat. 19 (2007) 315207.

218. G.X. Miao, A. Gupta, H. Sims, W.H. Butler, S. Ghosh, and G. Xiao, J. Appl. Phys. 97 (2005) 10C924.

219. G.X. Miao, P.LeClair, A. Gupta, G. Xiao, M. Varela, and S. Pennycook, Appl. Phys. Lett. 89 (2006) 022511.

220. P. Seneor, A. Fert, J.-L. Maurice, F. Montaigne, F. Petroff, and A. Vaurès, Appl. Phys. Lett. 74 (1999) 4017.

221. K. Ghosh, S.B. Ogale, S.P. Pai, M. Robson, E. Li, I. Jin, Z.-W. Dong, R.L. Greene, R. Ramesh, and T. Venkatesan, Appl. Phys. Lett. 73 (1998) 689.

222. Y.F. Chen, M. Ziese, J. Phys. D: Appl. Phys. 40 (2007) 3271.

223. G. Hu and Y. Suzuki, Phys. Rev. Lett. 89 (2002) 276601.

224. L.M.B. Alldredge, R.V. Chopdekar, B.B. NelsonCheeseman, Y. Suzuki, Appl. Phys. Lett. 89 (2006) 182504.

225. E.Y. Tsymbal and D.G. Pettifor, J. Phys.: Cond. Matt. 9 (1997) L411.

226. J.M. MacLaren, X.-G. Zhang, W.H. Butler, X.-D. Wang, Phys. Rev. B 59 (1999) 5470.

227. C. Tiusan, J. Faure-Vincent, C. Bellouard, M. Hehn, E. Jouguelet, and A. Schuhl, Phys. Rev. Lett. 93 (2004) 106602.

228. K.D. Belashchenko, J.P. Velev, and E.Y. Tsymbal, Phys. Rev. B 72 (2005) 140404(R).

229. J. Mathon and A. Umerski, Phys. Rev. B 71 (2005) 220402.

230. X.-G. Zhang, W.H. Butler, and A. Bandyopadhyay, Phys. Rev. B 68 (2003) 092402.

231. J.D. Burton, S.S. Jaswal, E.Y. Tsymbal, O.N. Mryasov, and O.G. Heinonen, Appl. Phys. Lett. 89 (2006) 142507.

232. C. Tusche, H. Meyerheim, N. Jedrecy, G. Renaud, A. Ernst, J. Henk, P. Bruno, and J. Kirschner, Phys. Rev. Lett. 95 (2005) 176101.

233. J.P. Velev, K.D. Belashchenko, and E.Y. Tsymbal, Phys. Rev. Lett. 96 (2006) 119601.

234. C. Heiliger, P. Zahn, B.Y. Yavorsky, and I. Mertig, Phys. Rev. B 72 (2005) 180406; ibid. 73 (2006) 214441.

235. D. Monsma and S. Parkin, J. Appl. Phys. 77 (2000) 720 \& 883 .

236. E.Y. Tsymbal, I.I. Oleinik, and D.G. Pettifor, J. Appl. Phys. 87 (2000) 5230.

237. K.D. Belashchenko, E.Y. Tsymbal, I.I. Oleinik, M. van Schilfgaarde, Phys. Rev. B 71 (2005) 224422.

238. Q. Ge, S.J. Jenkins and D.A. King, Chem. Phys. Lett. 327 (2000) 125.

239. S.J. Jenkins, Q. Ge and D.A. King, Phys. Rev. B 64 (2001) 012413.
240. S. Yamagishi, S.J. Jenkins and D.A. King, J. Chem. Phys. 114 (2001) 5765.

241. S. Yamagishi, S.J. Jenkins, and D.A. King, J Am. Chem. Soc. 126 (2004) 10962.

242. S.J. Jenkins, Surf. Sci. 600 (2006) 1431.

243. P.K. de Boer, G.A. de Wijs, and R.A. de Groot, Phys. Rev. B 58 (1998) 15422.

244. I.I. Oleinik, E.Y. Tsymbal, and D.G. Pettifor, Phys. Rev. B 65 (2002) 020401(R).

245. D. Ristoiu, J.P. Nozières, C.N. Borca, B. Borca, and P.A. Dowben, Appl. Phys. Lett. 76 (2000) 2349.

246. J. Choi, J. Zhang, S.-H. Liou, P.A. Dowben, and E.W. Plummer, Phys. Rev. B 59 (1999) 13453.

247. G.L. Bona, F. Meier, M. Taborelli, E. Bucher, and P.H. Schmidt, Sol. St. Comm. 56 (1985) 391.

248. A.N. Caruso, C.N. Borca, D. Ristoiu, J.P. Nozières, and P.A. Dowben, Surf. Sci. 525 (2003) L109.

249. A.N. Caruso and C.N. Borca, Rec. Dev. Magn. Magn. Mater. 1 (2003) 33.

250. H. Dulli, P.A. Dowben, S.-H. Liou, and E.W. Plummer, Phys. Rev. B 62 (2000) 14629(R).

251. C.N. Borca, D. Ristoiu, Q. L. Xu, S.-H. Liou, S. Adenwalla, and P.A. Dowben, J. Appl. Phys. 87 (2000) 6104.

252. H. Dulli, E.W. Plummer, P.A. Dowben, J. Choi, and S.H. Liou, Appl. Phys. Lett. 77 (2000) 570.

253. C.N. Borca, B. Xu, T. Komesu, H.-K. Jeong, M.T. Liu, S.-H. Liou, S. Stadler, Y. Idzerda, and P.A. Dowben, Europhys. Lett. 56 (2001) 722.

254. C.N. Borca, B. Xu, T. Komesu, H.-K. Jeong, M.T. Liu, S.-H. Liou, and P.A. Dowben, Surf. Sci. 512 (2002) L346.

255. J. Choi, C. Waldfried, S.-H. Liou, and P.A. Dowben, J. Vac. Sci. Tech. A 16 (1998) 2950.

256. W. Zhang, X. Wang, and I. Boyd, Appl. Phys. Lett. 73 (1998) 2745.

257. L.-C. Dufour, G.L. Bertrand, G. Caboche, P. Decorse, A. El Anssari, A. Poirson, and M. Vareille, Sol. St. Ionics 101 (1997) 661.

258. J. Choi, H. Dulli, Y. Feng, S.-H. Liou, P.A. Dowben, and M.A. Langell, Phys. Stat. Sol. (b) 214 (1999) 45.

259. J. Zhang, D.N. McIlroy, P.A. Dowben, S.-H. Liou, R.F. Sabirianov, and S.S. Jaswal, Sol. St. Comm. 97 (1996) 39.

260. C. Waldfried, D.N. McIlroy, S.-H Liou, R. Sabiryanov, S.S. Jaswal, and P.A. Dowben, J. Phys.: Cond. Matt. 9 (1997) 1031.

261. D.N. McIlroy, C. Waldfried, J. Zhang, J. Choi, F. Foong, S.-H. Liou, and P.A. Dowben, Phys. Rev. B 54 (1996) 17438.

262. M. Yoshimoto, H. Maruta, T. Ohnishi, K. Sasaki, and H. Koinuma, Appl. Phys. Lett. 73 (1998) 187.

263. M. Izumi, Y. Konishi, T. Nishihara, S. Hayashi, M. Shinohara, M. Kawasaki, and Y. Tokura, Appl. Phys. Lett. 73 (1998) 2497. 
264. A.A. Zakharov, H. Nylén, M. Quavford, I. Lindau, M. Leandersson, M.B. Tsetlin, and M.N. Mikheeva, Phys. Rev. B 56 (1997) 9030.

265. J. Dai, J. Tang, H. Xu, L. Spinu, W. Wang, K.-Y. Wang, A. Kumbhar, M. Li and U. Diebold, Appl. Phys. Lett. 77 (2000) 2840.

266. R. Cheng, B. Xu, C.N. Borca, A. Sokolov, C.-S. Yang, L. Yuan, S.-H. Liou, B. Doudin and P.A. Dowben, Appl. Phys. Lett. 79 (2001) 3122.

267. N.D. Telling, G. van der Laan, S. Ladak and R.J. Hicken, Appl. Phys. Lett. 85 (2004) 3803.

268. D. Lee and J. Hong, J. Appl. Phys. 97 (2005) 093905.

269. R. de Masi, D. Reinicke, F. Muller, P Steiner and S. Hufner, Surf. Sci. 515 (2002) 523.

270. H.L. Meyerheim, R. Popescu, J. Kirscher, N. Jedrecy, M. Sauvage-Simkin, B. Heinrich, and R. Pinchaux, Phys. Rev. Lett. 87 (2001) 076102.

271. J. Hong, Y. Lee, M.K. Lee, H.J. Song, H.-J. Shin, Y. Yoo, and J. Suh, Appl. Phys. Lett. 83 (2003) 4803.

272. H. Ohldag, T.J. Regan, J. Stohr, A. Scholl, F. Nolting, J. Luning, C. Stamm, S. Anders, and R.L. White, Phys. Rev. Lett. 87 (2001) 247201.

273. H. Ohldag, A. Scholl, F. Nolting, S. Anders, F.U. Hillebrecht, and J. Stohr, Phys. Rev. Lett. 86 (2001) 2878.

274. T.J. Regan, H. Ohldag, C. Stamm, F. Nolting, J. Luning, J. Stohr, and R.L. White, Phys. Rev. B 64 (2001) 214422.

275. S. Colonna, P. Luches, S. Valeri, and F. Bosherini, Nuc. Inst. Meth. Phys. Res. B 246 (2006) 131.

276. R.F.C. Farrow, M.J. Carey, R.F. Marks, P.M. Rice, and D.J. Smith, Appl. Phys. Lett. 77 (2000) 1191.

277. J. Schmalhorst, S. Kammerer, M. Sacher, G. Reiss, A. Hutten, and A. Scholl, Phys. Rev. B 70 (2004) 024426.

278. H. Ohldag, A. Scholl, F. Nolting, E. Arenholz, S. Maat, A.T. Young, M. Carey, and J. Stohr, Phys. Rev. Lett. 91 (2003) 017203.

279. K. Takano, R.H. Kodama, A.E. Berkowitz, W. Cao, and G. Thomas, Phys. Rev. Lett. 79 (1997) 1130.

280. M. Finazzi, Phys. Rev. B 69 (2004) 064405.

281. P. Luches, V. Bellini, S. Colonna, L. Di Giustino, F. Manghi, S. Valeri, and F. Boscherini, Phys. Rev. Lett. 96 (2006) 106106.

282. M. Bowen, V. Cros, H. Jaffres, P. Bencok, F. Petroff, and N. B. Brookes, Phys. Rev. B 73 (2006) 012405.

283. N.D. Telling, G van der Laan, S. Laak, R.J. Hicken, and E. Arenholz, J. Appl. Phys. 99 (2006) 08E505.

284. J. Schmahorst, M. Sacher, A. Thomas, H. Bruckl, G. Reiss, and K. Stark, J. Appl. Phys. 97 (2005) 123711.

285. I.I. Oleinik, E.Y. Tysmbal and D.G. Pettifor, Phys. Rev. B 62 (2000) 3952.

286. C. Li and A. J. Freeman, Phys. Rev. B 43 (1991) 780.

287. X.-G. Zhang and W.H. Butler, Phys. Rev. B 70 (2004) 172407.
288. J.S. Moodera, E.F. Gallagher, K. Robinson, and J. Nowak, Appl. Phys. Lett. 70 (1997) 3050.

289. M.G. Garnier, T. de los Arcos, J. Boudaden, and P. Oelhafen, Surf. Sci. 536 (2003) 130.

290. Handbook of Chemistry and Physics, 85th Edition, David Lide (ed), (CRC Press, Boca Raton, 2005).

291. M. Finazzi, A. Brambilla, L. Duo, G. Ghiringhelli, M. Portalupi, F. Ciccacci, M. Zacchingna and $\mathrm{M}$. Zangrando, Phys. Rev. B 70 (2004) 235420.

292. S. Benedetti, P. Luches, M. Liberati, and S. Valeri, Surf. Sci. 572 (2004) L348.

293. P.A. Dowben and A. Miller, Surface Segregation Phenomena, CRC Press, Boca Raton (1990).

294. H.-K. Jeong, A. Caruso, and C. Borca, Half Metallic Alloys: Fundamentals and Applications 676 (2005) 221.

295. C.N. Borca, D. Ristoiu, H.-K. Jeong, T. Komesu, A.N. Caruso, J. Pierre, L. Ranno, J.P. Nozieres, and P.A. Dowben, J. Phys.: Cond. Mat. 31 (2007) 315211.

296. W. Grentz, Diplom Arbeit, Free Universität Berlin (1991).

297. R. Cheng, C.N. Borca, N. Pilet, B. Xu, L. Yuan, B. Doudin, S.H. Liou, and P.A. Dowben, Appl. Phys. Lett. 81 (2002) 2109.

298. J. Schmalhorst, S. Kammerer, M. Sacher, G. Reiss, A. Hutten and A. Scholl, Phys. Rev. B 70 (2004) 24426.

299. A. Hutten, S. Kammerer, J. Schmalhorst, and G. Reiss, Half-metallic Alloys: Fundamentals and Applications, Springer (2006) 241.

300. D. Orgassa, H. Fujiwara, T.C. Schulthess, and W.H. Butler, Phys. Rev. B 60 (1999) 13237.

301. D. Orgassa, H. Fujiwara, T.C. Schulthess, and W.H. Butler, J. Appl. Phys. 87 (2000) 5870.

302. S. Picozzi, A. Continenza, and A. J. Freeman, Halfmetallic Alloys: Fundamentals and Applications, Springer (2006) 41.

303. M. Raphael, B. Ravel, M. Willard, S. Cheng, B. Das, R. Stroud, K. Bussmann, J. Claassen, and V. Harris, Appl. Phys. Lett. 79 (2001) 4396.

304. H. J. Elmers, H. G. Fecher, D. Valdaitsev, S. A. Nepijko, A. Gloskovskii, G. Jakob, G. Schonhense, S. Wurmehl, T. Block, C. Felser, P.-C. Hsu, W.-L Tsai, and S. Cramm, Phys. Rev. B 67 (2003) 104412.

305. C. Felser, H. J. Elmers, and G. H. Fecher, Half-metallic Alloys: Fundamentals and Applications, Springer (2006) 113.

306. S.J. Jenkins, Phys. Rev. B 70 (2004) 245401.

307. S.J. Jenkins, in Local Moment Ferromagnets, edited by M. Donath and W. Nolting (2004) 245.

308. B. Alling, S. Shallcross, I.A. Abrikosov Phys. Rev. B 73 (2006) 064418.

309. J.J. Attema, C.M. Fang, L. Chioncel, G.A. de Wijs, A.I. Lichtenstein, R.A. de Groot, J.Phys. Cond. Mat. 16 (2004) S5517. 
310. W. Wulfhekel, M. Klaua, D. Ullmann, F. Zavaliche, J. Kirschner, R. Urban, T. Monchesky, and B. Heinrich, Appl. Phys. Lett. 78 (2001) 509.

311. P. Mather, J. Read, and R. Buhrman, Phys. Rev. B 73 (2006) 205412.

312. J.P. Velev, K. Belashchenko, S.S. Jaswal, and E.Y. Tsymbal, Appl. Phys. Lett. 90 (2007) 072502.

313.E.Y. Tsymbal and D.G. Pettifor, Phys. Rev. B 58 (1998) 432; J. Appl. Phys. 85 (1999) 5801.

314.H. Itoh et al., J. Phys. Soc. Jap. 68 (1999) 1632.

315.E.Y. Tsymbal and D.G. Pettifor, Phys. Rev. B 64 (2001) 212401.

316. E.Y. Tsymbal, A. Sokolov, I.F. Sabirianov, and B. Doudin, Phys. Rev. Lett. 90 (2003) 186602.

317. A. Sokolov, C.-S. Yang, L. Yuan, S.-H. Liou, R. Cheng, H.-K. Jeong, T. Komesu, B. Xu, C.N. Borca, P.A. Dowben and B. Doudin, Europhys. Letters 58 (2002) 448.

318. A. Sokolov, C.-S. Yang, L. Yuan, S.-H. Liou, R. Cheng, B. Xu, C.N. Borca, P.A. Dowben and B. Doudin, J. App. Phys. 91 (2002) 8801.

319. J.P. Velev, M.Ye. Zhuravlev, K.D. Belashchenko, S.S. Jaswal, E.Y. Tsymbal, T. Katayama, and S. Yuasa, IEEE Tran. Magn. 43 (2007) 2770.

320. I.J. Vera Marún, F.M. Postma, J.C. Lodder, and R. Jansen, Phys. Rev. B 76 (2007) 064426.

321. R. Cheng, A.N. Caruso, L. Yuan, S.-H. Liou, and P.A. Dowben, Appl. Phys. Lett. 82 (2003) 1443.

322. H. Yanagihara, Y. Toyoda, E. Kita, J. Appl. Phys. 101 (2007) 09D101.

323. D.D. Fong et al., Science 304 (2004) 1650.

324. C. Lichtensteiger et al., Phys. Rev. Lett. 94 (2005) 047603.

325. D.A. Tenne et al., Science 313 (2006) 1614.

326. J. Junquera and $\mathrm{Ph}$. Ghosez, Nature (London) 422 (2003) 506.

327. N. Sai, A.M. Kolpak, and A.M. Rappe, Phys. Rev. B 72 (2005) 020101.

328. G. Gerra, A.K. Tagantsev, N. Setter, and K. Parlinski, Phys. Rev. Lett. 96 (2006) 107603.

329. K.M. Rabe, C.H. Ahn, and J.-M. Triscone (Eds.), Physcs of ferroelectrics, Springer, Berlin 2007.

330. J. Rodríguez Contreras, Dissertation der Universität zu Köln (2004).

331. J. Xiao, A. Sokolov, P.A. Dowben, Appl. Phys. Lett. 90 (2007) 242907.

332. L.M. Blinov, V.M. Fridkin, S.P. Palto, A.V. Bune, P.A. Dowben, and S. Ducharme, Uspekhi Fiz. Nauk Russ. Ed.. 170 (2000) 247; Phys. Uspekhi Eng. Ed.. 43 (2000) 243.

333. W.F. Brinkman, R.C. Dynes, and J.M. Rowell, J. Appl. Phys. 41 (1970) 1915.

334. Y.D. Xia, W.Y. He, L. Chen, X.K. Meng, Z.G. Liu, Appl. Phys. Lett. 90 (2007) 022907.

335. H. Akazawa, Jpn. J. Appl. Phys. 46 (2007) L848.
336. M. P. Singh, L. Méchin, W. Prellier, M. Maglione, Appl. Phys. Lett. 89 (2006) 202906.

337. C.-G. Duan, R. F. Sabirianov, W.-N. Mei, S. S. Jaswal, and E.Y. Tsymbal, Nano Lett. 6 (2006) 483.

338. C.-G. Duan, S.S. Jaswal, and E.Y. Tsymbal, Phys. Rev. Lett. 97 (2006) 047201.

339. G. Gerra, A.K. Tagantsev, and N. Setter, Phys. Rev. Lett. 98 (2007) 207601.

340. J.P. Velev, C.-G. Duan, K.D. Belashchenko, S.S. Jaswal, and E.Y. Tsymbal, Phys. Rev. Lett. 98 (2007) 137201.

341. J.P. Velev, C.-G. Duan, K.D. Belashchenko, S.S. Jaswal, and E.Y. Tsymbal, J. Appl. Phys. 103 (2008) 07A701.

342. M.Ye. Zhuravlev, S.S. Jaswal, E.Y. Tsymbal, and R.F. Sabirianov, Appl. Phys. Lett. 87 (2005) 222114.

343. S. Sahoo, S. Polisetty, C.-G. Duan, S.S. Jaswal, E.Y. Tsymbal, and Ch. Binek, Phys. Rev. B 76 (2007) 092108.

344. C.-G. Duan, J. Velev, R. Sabirianov, W. Mei, S. Jaswal, and E. Y. Tsymbal, Appl. Phys. Lett. 92 (2008) 122905.

345. Y.H. Chu, L.W. Martin, M.B. Holcomb, and R. Ramesh, Mater. Today 10 (2007) 16.

346. P. Borisov, A. Hochstrat, X. Chen, W. Kleemann, and C. Binek, Phys. Rev. Lett. 94 (2005) 117203.

347. Y. Chen, T. Sakai, T. Chen, S.D. Yoon, A.L. Geiler, C. Vittoria, and V.G. Harris, Appl. Phys. Lett. 88 (2006) 062516.

348. U. Laletsin, N. Paddubnaya, G. Srinivasan, M.I. Bichurin, cond-mat/0401648.

349. A. Ustinov, G. Srinivasan, and B. A. Kalinikos, J. Appl. Phys. 101 (2007) 09M503.

350. J. Das, B.A. Kalinikos, A.R. Barman, and C.E. Patton, Appl. Phys. Lett. 91 (2007) 172516.

351. Z.H. Xiong, D. Wu, Z.V. Vardeny and Jing Shi, Nature 427 (2004) 821.

352. J.R. Petta, S.K. Slater, and D.C. Ralph, Phys. Rev. Lett. 93 (2004) 136601.

353. W. Xu, G.J. Szulczewski, P. LeClair, I. Navarrete, R. Schad, G. Miao, H. Guo, and A. Gupta, Appl. Phys. Lett. 90 (2007) 72506.

354. J.S. Miller, Inorg. Chem. 39 (2000) 4392.

355. R. Sessoli, D. Gatteschi, A. Caneschi, and M.A. Novak, Nature 365 (1993) 141.

356. J.S. Miller, J.C. Calabrese, H. Rommelmann, S. Chittipeddi, A.J. Epstein, J.H. Zhang, and W.M. Reiff, J. Am. Chem. Soc. 109 (1987) 769.

357. J.M. Manriquez, G.T. Yee, R.S. McLean, A.J. Epstein, and J.S. Miller, Science 252 (1991) 1415.

358. S. Ferlay, T. Mallah, R. Ouahes, P. Veillet, and M. Verdauger, Nature 378 (1995) 701.

359. V.N. Prigodin, N.P. Raju, K.I. Pokhodnya, J.S. Miller, and A.J. Epstein, Adv. Mater. 14 (2002) 1230.

360. V.N. Prigodin, N.P. Raju, K.I. Pokhodnya, J.S. Miller, A.J. Epstein, Synth. Metals 135-136 (2003) 87. 
361. N.P. Raju, T. Savrin, V.N. Prigodin, K.I. Pokhodnya, J.S. Miller, A.J. Epstein, J. Appl. Phys. 93 (2003) 6799.

362. V.N. Prigodin, J.D. Bergeson, D.M. Lincoln, A.J. Epstein, Synth. Metals 156 (2006) 757.

363. A.J. Epstein, US Patent No. 7075815 (2006).

364. K.I. Pokhodnya, A.J. Epstein, and J.S. Miller, Adv. Mat. $12(2000) 410$.

365. S.J. Luo and K.L. Yao, Phys. Rev. B 67 (2003) 214429.

366. M.-H. Wu and W.-D. Zou, J. Math. Chem. 40 (2006) 319.

367. R. Arita, Y. Suwa, K. Kuroki, and H. Aoki, Phys. Rev. Lett. 88 (2002) 127202.

368. D.H. Kim, J.S Yang, K.W. Lee, S.D. Bu, T.W. Noh, S.J. Oh, Y.W. Kim, J.S. Chung, H. Tanaka, H.Y Lee, and T. Kawai, Appl. Phys. Lett. 81 (2002) 2421.

369. T.S. Santos, J.S. Lee, P. Migdal, I.C. Lekshmi, B. Satpati, and J.S. Moodera, Phys. Rev. Lett. 98 (2007) 16601.

370. A. Ohtomo and H. Y. Hwang, Nature 427 (2004) 423.

371. A. Ohtomo, D. A. Muller, J. L. Grazul, and H. Y. Hwang, Nature 419 (2002) 378.

372. S. Thiel et al., Science 313 (2006) 1942.

373. A. Brinkman et al., Nature Mater. 6 (2007) 493.

374. N. Nakagawa, H. Y. Hwang, and D. A. Muller, Nature Mater. 5 (2006) 204.

375. Z.S. Popovich and S. Satpathy, Phys. Rev. Lett. 94 (2005) 176805.

376. M.S. Park, S.H. Rhim, and A.J. Freeman, Phys. Rev. B 74 (2006) 205416.

377. R. Pencheva and W. E. Pickett, Phys. Rev. B 74 (2006) 035112; Phys. Rev. Lett. 99 (2007) 016802.

378. S. Okamoto, A.J. Millis, and N.A. Spaldin, Phys. Rev. Lett. 97 (2006) 056802.

379. M. Basletic, J.-L. Maurice, C. Carrétéro, G. Herranz, O. Copie, M. Bibes, É. Jacquet, K. Bouzehouane, S. Fusil, A. Barthélémy, preprint.

380. K. Janicka, J.P. Velev, and E.Y. Tsymbal, J. Appl. Phys. 103 (2008) 07B508. 